\title{
Cryptic subterranean diversity in Collembola indicates that Hot Spot in ferruginous rock may have serious implications in Brazilian conservation policy.
}

\section{Douglas Zeppelini ( $\square$ zeppelini@daad-alumni.de )}

Coleção de Referência de Fauna de Solo, Universidade Estadual da Paraíba João Victor L. C. Oliveira

Universidade Federal da Paraíba

\section{Estevam C. A. Lima}

Coleção de Referência de Fauna de Solo, Universidade Estadual da Paraíba

Roniere A. Brito

Coleção de Referência de Fauna de Solo, Universidade Estadual da Paraíba

\section{Aila S. Ferreira}

Coleção de Referência de Fauna de Solo, Universidade Estadual da Paraíba

\section{Luis C. Stievano}

Coleção de Referência de Fauna de Solo, Universidade Estadual da Paraíba

\section{Nathan P. Brito}

Coleção de Referência de Fauna de Solo, Universidade Estadual da Paraíba

\section{Misael A. Oliveira-Neto}

Coleção de Referência de Fauna de Solo, Universidade Estadual da Paraíba

\section{Bruna C. H. Lopes}

Universidade Federal da Paraíba

\section{Research Article}

Keywords: Diversity, Iron Caves, MSS, Cave Collembola, New Species

Posted Date: November 12th, 2021

DOI: https://doi.org/10.21203/rs.3.rs-1051939/v1

License: (c) (1) This work is licensed under a Creative Commons Attribution 4.0 International License. Read Full License 
Cryptic subterranean diversity in Collembola indicates that Hot Spot in ferruginous rock may have serious implications in Brazilian conservation policy.

Douglas Zeppelini ${ }^{1,2, *}$, João Victor L. C. Oliveira ${ }^{1,2}$, Estevam C. Araújo de Lima ${ }^{1}$, Roniere A. Brito $^{1}$, Aila S. Ferreira ${ }^{1}$, Luis C. Stievano ${ }^{1}$, Nathan P. Brito ${ }^{1}$, Misael A. Oliveira-Neto ${ }^{1}$, and Bruna C. H. Lopes ${ }^{1,2}$

${ }^{1}$ Laboratório de Sistemática de Collembola e Conservação - Coleção de Referência de Fauna de Solo - CCBSA - Universidade Estadual da Paraíba campus V, João Pessoa, PB, Brazil, 58070-450. ${ }^{2}$ Programa de Pós-graduação em Ciências Biológicas - Zoologia, Universidade Federal da Paraíba, João Pessoa, Paraíba, Brazil.

*Corresponding Author: zeppelini@daad-alumni.de

\begin{abstract}
A super diverse hot spot of subterranean Collembola in ferruginous rock caves and Mesovoid Shallow Substratum is revealed by the analysis of cryptic diversity. The diversity is accessed by detailed description of chaetotaxy and slight variation in morphology of 11 new species of Trogolaphysa Mills, 1938 (Collembola, Paronellidae, Paronellinae) and the 49 previously recorded species of springtails from caves, using optical and electronic microscopy. When combined with recent subterranean surveys, our results show an important reservoir of cave diversity in the Mesovoid Shallow Substratum, contrasting with the conservation policy for subterranean fauna in metallogenic areas in Brazil which prioritizes the caves instead the cave species, which may be extremely detrimental to the fauna in the shallow subterranean habitats not accessible to humans.
\end{abstract}

Key words: Diversity, Iron Caves, MSS, Cave Collembola, New Species 


\section{Introduction}

Some areas are subject of intense fauna diversification, the term "hot spot" is used to indicate relatively small areas with high and exclusive diversity, though there are different interpretations about what is the threshold which defines such an area. Hot spots may be defined by combining the richness, endemism, extension, and threats to the area in focus [1], however some approaches to subterranean fauna use an arbitrary cutoff of 20 restricted endemic species with no regards to environmental threats [2]. More recently two South American subterranean hot spots were defined based in the richness of restricted endemic fauna, and fully addressed the conservation aspects of the surroundings of the cave systems [3].

The species diversity of cave restricted fauna, mostly troglobites, is positively correlated to the extension of the cave and the presence of perennial pools, and sometimes negatively correlated to the presence of streams, which can cause disturbance in the habitats, and import a more diverse troglophile fauna [4]. Therefore, the more diverse troglobitic fauna is supposed to be found in larger caves, which are often formed in limestone rock, this is corroborated by the cave fauna hot spots found in limestone cave systems in Southeastern and Northeastern Brazil $[3,5]$.

Nevertheless, there are subterranean spaces that extend through and across the weathered rock matrix, named Mesovoid Shallow Substratum (MSS) [6,7]. These underground spaces connect, and somehow extends the cave habitat far beyond the human reach and might be a climate refuge to epigeic fauna [8].

The Brazilian States of Minas Gerais (Southeast) and Para (North) represent the more important metallogenic areas in the country and concentrate the mining activities and commodities production. The iron ore lithology presents a profusion of small and shallow caves, subterranean spaces and crevices that functions as a real MSS [9], providing habitat for a variety of species, including troglobites $[10,11]$. Previous studies found higher average relative richness, and distinctiveness in ferruginous rock [9], than in other lithologies.

Unpublished data from caves and MSS in ferruginous rock, brings 87 undescribed species of Collembola with some degree of troglomorphism from Minas Gerais (73) and Para (14), a total of 37 species have been described so far (31 from Minas Gerais, six from Para), including 21 troglobites, among which 19 species in ferruginous cave and MSS (Table 1). Recent studies in Brazil have surveyed hundreds of cave species, from sponges to vertebrates, more than 250 already described $[3,11,12,13,14]$. Great part of the subterranean biology research focuses on large caves in karstic lithology $[15,16]$.

Here we present a group of 11 new species of Collembola of the genus Trogolaphysa Mills, 1938 with some degree of troglomorphism, from caves and MSS in ferruginous and limestone rock. The genus Trogolaphysa has 69 described species worldwide, only eight have been recorded from 
Brazil: T. aelleni Yoshii, 1988; T. ernesti Cipola \& Bellini, 2017; T. formosensis Silva \& Bellini, 2015; T. hauseri Yoshii, 1988; T. hirtipes (Handschin, 1924), T. millsi Arlé, 1939; T. piracurucaensis Nunes \& Bellini, 2018; and T. tijucana (Arlé \& Guimarães, 1979). Our results depict an important hot spot for cave Collembola in the State of Minas Gerais, also corroborate the expected high species richness in ferruginous rock caves and MSS and shed some light to the impact of the MSS in the conservation policy as a refuge for subterranean diversity.

\begin{tabular}{|c|c|c|c|}
\hline Species, Author Year & $\begin{array}{l}\text { Ecological } \\
\text { status* }\end{array}$ & Lithology & State \\
\hline Acherontides eleonorea Palacios-Vargas \& Gnaspini-Netto, 1992 & Troglobite & $\mathrm{Li}^{1}$ & SP \\
\hline Acherontides serrasapoensis Lima, Stievano \& Zeppelini, 2019 & Troglophile & $\operatorname{Ir}^{1,2,3}$ & MG \\
\hline Arrhopalites mendoncae Brito, Lima \& Zeppelini, 2019 & Troglobite & $\mathrm{Ir}, \mathrm{Li}^{1}$ & MG \\
\hline Arrhopalites alambariensis Zeppelini, 2006 & Troglobite & $\mathrm{Li}^{1}$ & SP \\
\hline Arrhopalites amorimi Palacios-Vargas \& Zeppelini, 1995a & Troglobite & $\mathrm{Li}^{1}$ & SP \\
\hline Arrhopalites botuveraensis Zeppelini, 2006 & Troglobite & $\mathrm{Li}^{1}$ & $\mathrm{SC}$ \\
\hline Arrhopalites glabrofasciatus Zeppelini, Brito \& Lima, 2018 & Troglobite & $\mathrm{Ir}, \mathrm{Li}^{1}$ & MG \\
\hline Arrhopalites gnaspinii Palacios-Vargas \& Zeppelini, 1995a & Troglobite & $\mathrm{Li}^{1}$ & SP \\
\hline Arrhopalites heteroculatus Zeppelini, 2006 & Troglobite & $\mathrm{Li}^{1}$ & SP \\
\hline Arrhopalites lawrencei Palacios-Vargas \& Zeppelini, 1995a & Troglobite & $\mathrm{Li}^{1}$ & SP \\
\hline Arrhopalites paranaenses Zeppelini, 2006 & Troglobite & $\mathrm{Li}^{1}$ & PR \\
\hline Coecobrya phoenix Brito, Lima \& Zeppelini, 2019 & Troglobite & $\mathrm{Ir}, \mathrm{Li}^{1}$ & MG \\
\hline Cyphoderus caetetus Zeppelini \& Oliveira, 2016 & Troglophile & $\operatorname{Ir}^{1,2,3}$ & MG \\
\hline Cyphoderus mucrominimus Oliveira, Alves \& Zeppelini, 2017 & Troglophile & $\operatorname{Ir}^{1}$ & PA \\
\hline Cyphoderus mucrostrimenus Oliveira, Alves \& Zeppelini, 2017 & Troglophile & $\operatorname{Ir}^{1,2,3}$ & PA \\
\hline Cyphoderus palaciosi Oliveira, Brito \& Zeppelini, 2021 & Troglophile & $\operatorname{Ir}^{1,2,3}$ & MG \\
\hline Cyphoderus pataxo Oliveira, Brito \& Zeppelini, 2021 & Troglophile & $\operatorname{Ir}^{1,2,3}$ & MG \\
\hline Pararrhopalites papaveroi (Zeppelini \& Palacios-Vargas, 1999) & Troglobite & $\mathrm{Li}^{1}$ & MS \\
\hline Pararrhopalites queirozi Brito, Lima \& Zeppelini, 2019 & Troglobite & $\mathrm{Li}^{1,2}$ & MG \\
\hline Pararrhopalites ubiquum Zeppelini, Lima \& Brito, 2018 & Troglobite & $\operatorname{Ir}^{1}$ & MG \\
\hline Pararrhopalites wallacei (Palacios-Vargas \& Zeppelini, 1995a) & Troglobite & $\mathrm{Li}^{1}$ & SP \\
\hline Pseudosinella acantholabrata Cipola, 2020 & Troglophile & $\operatorname{Ir}^{1,3}$ & MG \\
\hline Pseudosinella alfanjeunguiculata Bellini, Cipola \& Souza, 2020 & Troglobite & $\operatorname{Ir}^{1}$ & MG \\
\hline Pseudosinella ambigua Zeppelini, Brito \& Lima, 2018 & Troglobite & $\mathrm{Li}^{1}$ & MG \\
\hline Pseudosinella aphelabiata Bellini, Cipola \& Souza, 2020 & Troglobite & $\operatorname{Ir}^{1}$ & MG \\
\hline Pseudosinella brumadinhoensis Cipola, 2020 & Troglobite & $\operatorname{Ir}^{2}$ & MG \\
\hline Pseudosinella chimerambigua Oliveira, Lima \& Cipola, 2020 & Troglobite & $\operatorname{Ir}^{1,2}$ & MG \\
\hline Pseudosinella diamantinensis Bellini, Cipola \& Souza, 2020 & Troglobite & $\operatorname{Ir}^{1}$ & MG \\
\hline Pseudosinella guanhaensis Zeppelini, Brito \& Lima, 2018 & Troglobite & $\mathrm{Gr}^{1}$ & MG \\
\hline Pseudosinella keni Cipola, 2020 & Troglobite & $\operatorname{Ir}^{1}$ & MG \\
\hline Pseudosinella labiociliata Cipola, 2020 & Troglobite & $\operatorname{Ir}^{1}$ & MG \\
\hline Pseudosinella labruspinata Cipola, 2020 & Troglobite & $\operatorname{Ir}^{1,2}$ & MG \\
\hline Pseudosinella macrolignicephala Oliveira, Lima \& Cipola, 2020 & Troglophile & $\operatorname{Ir}^{1,2,3}$ & MG \\
\hline Pseudosinella marianensis Bellini, Cipola \& Souza, 2020 & Troglophile & $\operatorname{Ir}^{1,3}$ & MG \\
\hline Pseudosinella mitodentunguilata Bellini, Cipola \& Souza, 2020 & Troglobite & $\operatorname{Ir}^{1}$ & MG \\
\hline Pseudosinella neriae Bellini, Cipola \& Souza, 2020 & Troglobite & $\operatorname{Ir}^{1}$ & MG \\
\hline Pseudosinella paraensis Cipola, 2020 & Troglobite & $\operatorname{Ir}^{1}$ & PA \\
\hline Pseudosinella parambigua Oliveira, Lima \& Cipola, 2020 & Troglophile & $\operatorname{Ir}^{1,2,3}$ & MG \\
\hline Pseudosinella phyllunguiculata Oliveira, Lima \& Cipola, 2020 & Troglobite & $\operatorname{Ir}^{1}$ & MG \\
\hline Pseudosinella prelabruscervata Oliveira, Lima \& Cipola, 2020 & Troglobite & $\operatorname{Ir}^{1}$ & MG \\
\hline
\end{tabular}


Pseudosinella pusilla Oliveira, Brito \& Cipola, 2020

Pseudosinella serpentinensis Cipola, 2020

Pseudosinella spurimarianensis Bellini, Cipola \& Souza, 2020

Pseudosinella taurina Cipola, 2020

Pseudosinella unimacrochaetosa Cipola, 2020

Troglobius brasiliensis Palacios-Vargas \& Zeppelini, 1995a

Troglobius ferroicus Zeppelini, Silva \& Palacios-Vargas, 2014

Trogolaphysa aelleni Yosii, 1988

Trogolaphysa barroca sp. nov.

Trogolaphysa bellinii sp. nov.

Trogolaphysa chapelensis sp. nov.

Trogolaphysa crystallensis sp. nov.

Trogolaphysa dandarae sp. nov.

Trogolaphysa epitychia sp. nov.

Trogolaphysa gisbertae sp. nov.

Trogolaphysa hauseri Yosii, 1988

Trogolaphysa lacerta sp. nov.

Trogolaphysa mariecurieae sp. nov.

Trogolaphysa sotoadamesi sp. nov.

$\begin{array}{lll}\text { Troglobite } & \mathrm{Ir}^{1} & \text { PA } \\ \text { Troglobite } & \mathrm{Ir}^{1} & \mathrm{MG} \\ \text { Troglophile } & \mathrm{Ir}^{1,2,3} & \mathrm{MG} \\ \text { Troglobite } & \mathrm{Ir}^{1} & \mathrm{PA} \\ \text { Troglophile } & \mathrm{Ir}^{1,3} & \mathrm{MG} \\ \text { Troglobite } & \mathrm{Sn}^{1} & \mathrm{PA} \\ \text { Troglobite } & \mathrm{Ir}^{1} & \mathrm{MG} \\ \text { Troglobite } & \mathrm{Li}^{1} & \mathrm{SP} \\ \text { Troglobite } & \mathrm{Ir}^{1} & \mathrm{MG} \\ \text { Troglobite } & \mathrm{Ir}^{1} & \mathrm{MG} \\ \text { Troglobite } & \mathrm{Ir}^{1} & \mathrm{MG} \\ \text { Troglobite } & \mathrm{Ir}^{1} & \mathrm{MG} \\ \text { Troglobite } & \mathrm{Ir}^{1} & \mathrm{PA} \\ \text { Troglobite } & \mathrm{Ir}^{1} & \mathrm{MG} \\ \text { Troglobite } & \mathrm{Ir}^{1} & \mathrm{PA} \\ \text { Troglobite } & \mathrm{Li}^{1} & \mathrm{SP} \\ \text { Troglobite } & \mathrm{Ir}^{1} & \mathrm{MG} \\ \text { Troglophile } & \mathrm{Ir}^{1}{ }^{1}, 2, & \mathrm{MG} \\ \text { Troglobite } & \mathrm{Ir}^{1} & \mathrm{MG} \\ \text { Troglobite } & \mathrm{Li}^{1} & \mathrm{SP}\end{array}$

Trogolaphysa zampauloi sp. nov.

Table 1: Collembola species described from caves and MSS in Brazil. Distributional States - Mato Grosso do Sul, MS. Minas Gerais, MG. Para, PA. Parana, PR. Santa Catarina, SC. São Paulo, SP. Lithology - Granitic rock, Gr. Iron rock, Ir. Limestone rock, Li. Sandstone, Sn. Cave ${ }^{1}, \mathrm{MSS}^{2}$, Surface $^{3}$ * We consider troglobite all species with some degree of troglomorphism and known distribution restricted to subterranean habitats (for a discussion on troglobite definition see Sket [17].

\section{Results}

This study presents 11 new species of cave Trogolaphysa (Table 1), two new species from Para, eight from Minas Gerais from caves and MSS in metallogenic rock, and one new species from Sao Paulo, found in a limestone cave. Species were collected directly from organic debris in caves, the MSS was accessed through samplings in drilling holes. These results represent an increase of $24.8 \%$ in the previous 31 species of cave Collembola described for the State of Minas Gerais. Data from Para are still scarce, with only five previous records from iron rock caves and one from sandstone cave (Table 1). The species from Sao Paulo is from a different lithology, a much larger cave with narrower connections to the MSS. It represents a new record to add to the 17 known cave springtails from limestone caves in Brazil (Table 1).

There are 49 valid species of cave Collembola previously recorded from Brazil, 34 troglobites and 15 troglophiles. For limestone caves, there are 17 species from five different States at South and Southeastern Regions, all troglobites. There is a troglobitic species from sandstone cave in the State of Para at Northern Region, and one troglobite from granitic caves in Minas Gerais. All the 33 remaining records are from ferruginous rock caves and MSS, 28 from the Southeastern State of Minas Gerais (18 troglobites and 10 troglophiles), and five from Para (two troglobites and three troglophiles). 
With the results presented here the total number of cave Collembola recorded for Brazil rises to 60 species, with 18 species from limestone caves, one species from sandstone cave, one from granitic cave, and iron caves and MSS with 43 known subterranean species. The State of Minas Gerais present the highest richness for cave Collembola in Brazil, with two species from limestone and granitic caves respectively, and 37 records from iron caves and MSS. All these species are endemic to ferruginous rock shallow caves and MSS in Minas Gerais, this has important implications for the conservation areas policy in Brazil and other ferruginous rock subterranean environments in tropical areas in the world.

\section{Discussion}

\section{Ferruginous Mesovoid Shallow Substratum}

The iron ore deposits in Brazil present a semi continuous covering layer of fragmented hematite and lesser components cemented by limonite, called Canga. It is formed by weathering and lixiviation, and produce a labyrinthic complex of subterranean spaces, crevices, and tiny underground connections, depicting a habitat that is analogous to the MSS [9].

In temperate zones the MSS plays a role as refuge for arthropod fauna, mainly at high altitudes where the cold weather can eliminate all the ectothermic fauna from the surface [8]. Similarly, seasonal migration movements are observed in the MSS for different taxa as response to hot dry summer [18,19]. When troglobitic fauna is concerned the MSS has a different role, cave restricted Collembola showed higher underground dispersal capacity than troglophiles [20], therefore, the MSS can connect neighboring caves systems and extend their distribution range.

In metallogenic rock, cave species richness is higher than in any other lithology [9]. The cave Collembola found in Brazil corroborates this assumption, from the total of 60 known cave species, one troglobite was recorded from sandstone caves and one from granitic cave, 18 species were recorded from limestone caves (all troglobites), and 43 species from iron caves and MSS (30 troglobites). Three troglobitic species were recorded from both limestone and iron caves, indicating potentially unrecognized cryptic species.

This is more relevant when considered that ferruginous rock represents only $0.15 \%$ of the Brazilian territory, nearly $12,000 \mathrm{~km}^{2}$ [21], and classic biospeleological research is focused on large (usually limestone) caves. The elevated richness of species restricted to small shallow caves, reinforces the role of the MSS as an extension of the cave environment, the diversity must be the result of local barriers related to the rocky outcrop and the surface phytophysiognomy.

The State of Minas Gerais is the most diverse with 39 species of cave Collembola, the complex mosaicist lithology and the ecotone Cerrado Forest-Atlantic Forest are the main barriers associated to the richness of species restricted to caves and MSS. In this State, the iron rock 
subterranean habitats host 26 troglobites, and provide habitat and refuge to 11 known troglophiles species.

For caves in non-ferruginous lithologies, the size and number of entrances influence the species richness by giving the surface fauna access to the subterranean environment, and as a sink for organic matter input [4]. Contrastingly the caves in iron rock are small and shallow, often with few meters of horizontal development, the connections to the MSS are visible and numerous. In this context, instead, the distribution of the troglobitic species suggests that the entrances of iron rock caves are the limits of the available subterranean habitat for troglobites inside-out, and of suitable habitats for troglophiles outside-in (Fig. 1). We can consider the entrances of these caves as windows of the MSS, the spatial limit of the subterranean environment which presents the minimum conditions to the survival of a troglobite, while partially inhibits the dispersion of troglophiles deeper in the MSS. Troglobites can disperse underground more efficiently than troglophiles, however troglophiles are more efficient than troglobites to disperse through the surface [20]. In ferruginous lithology the size of the cave and its entrance influences the species richness [4], mainly because large iron caves can greatly affect the capacity of collectors and biologists to access the troglobitic fauna in the MSS, as the number of accessible connections to the MSS increases exponentially with the length of the cave in iron rock [9].

Another contrast of ferruginous rock caves is that the biotrophic flow seems to be inverted (Fig. 1), in limestone caves the energy and fauna come from outside through the cave entrance, and the fauna eventually speciate to become troglobitic, possibly restricted to the depths of a single cave. Despite the demonstrated existence of an epikarst, the particularities of the weathering process, and the water percolation [22], limestone caves tend to be large and grow deep through dissolution of the rock by water during the genesis of the cave. The deeper the cave is, the lesser the permeability of the rock, the epikarst usually reaches bout $15 \mathrm{~m}$ deep [22].

In iron caves the fauna comes from the above ground through the MSS connections between surface and subterranean environments, the same happens with energy that comes with roots that reach the MSS abundantly [9]. The troglobites develop in the MSS and eventually reach the caves where it can be seen in its distribution limits, and the troglophiles go in the opposite direction, inhabiting the surface and going inside the caves to refuge from climate, but not going too far in the MSS (see Table 1, species marked with ${ }^{1,3}$ ). 

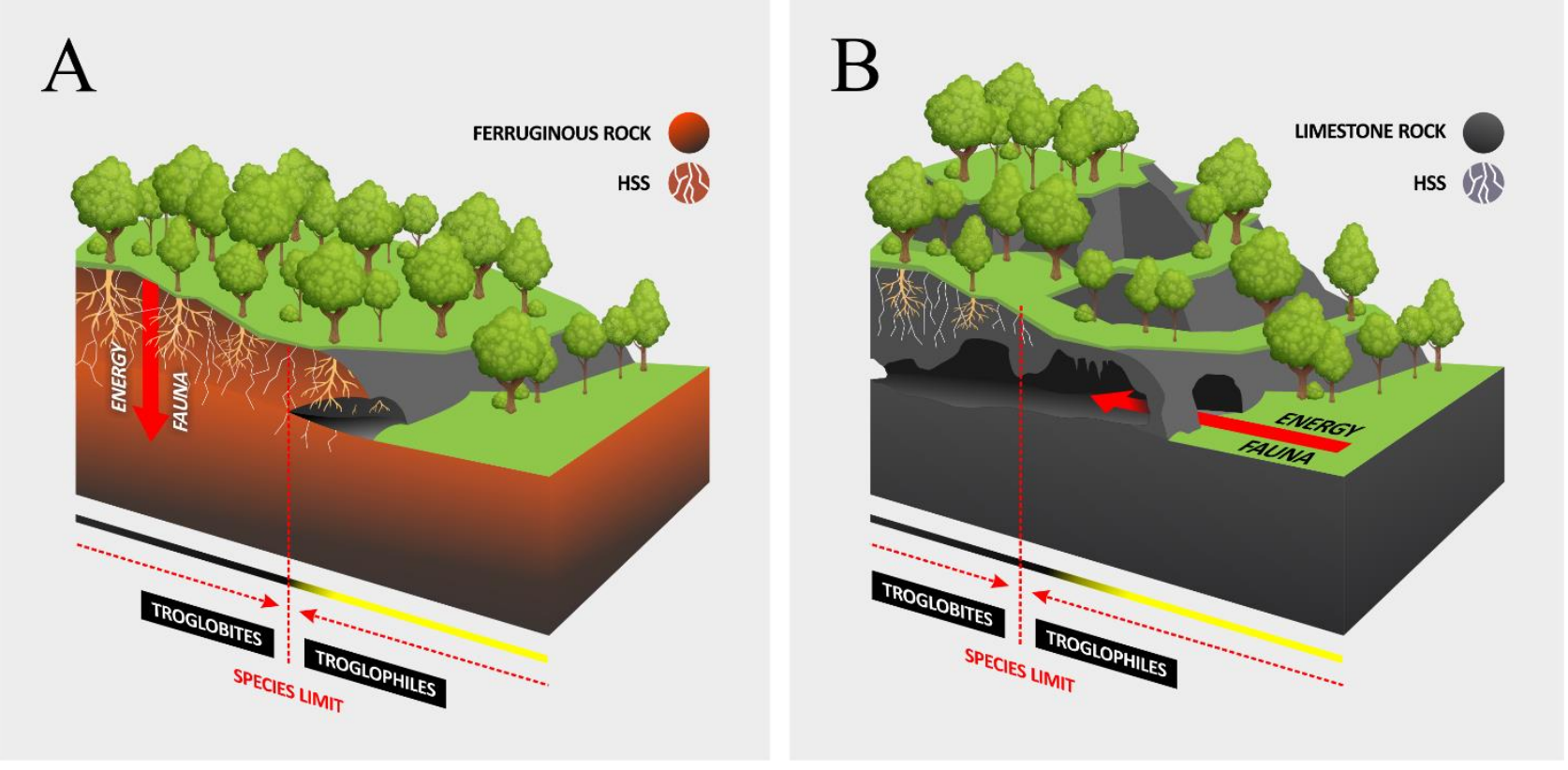

Figure 1. Schematic profile of ferruginous rock cave and limestone rock cave. A) Ferruginous rock - small and shallow caves, abundant roots, reticulated MSS; fauna and energy come from the above ground (solid red arrow), troglobites inhabit the MSS and reach the limits of the cave, troglophiles inhabit the surrounding and the cave, eventually reaching shortly in the MSS (dotted red arrow). B) Limestone rock - large caves, usually not reached by roots, sparse or absent MSS; fauna and energy come through the cave entrance (solid red arrow), troglobites inhabit the deep aphotic zone reaching the aphotic intermediary zone, troglophiles inhabit the surroundings and the cave, eventually reaching the deep aphotic zone. Yellow to black bar represents the light reach.

\section{Cryptic Diversity}

Large caves with deep aphotic zones, stable abiotic conditions, water pools, often hosting bat colonies, are correlated to high number of restricted species [9], usually displaying classic troglomorphism as absence of eyes and body pigments, elongated appendages, increased body size [23]. In the ferruginous rock MSS the limited available spaces impose selective pressures that drive the troglomorphism to a different direction. Collembola species restricted to the MSS and caves in iron rock tend to be reduced in size, with normal to shortened (even though always functional) appendages, reduction in eyes number and body pigments.

There are discrete morphological differences in cryptic species, to access this information it is necessary to expand the morphological refinement, some species are grouped together as result of limited selection of diagnostic characters. Cryptic species recognized from a single widespread species complex through barcode sequencing, revealed related morphological differences corresponding to the species separation [24].

The species found in ferruginous caves and MSS are very similar in most of its macro morphology, the differentiation is masked by homoplasy, and species recognition often must rely on details of chaetotaxy (Fig. 2-43) and slight variations of morphological structures, often overlooked, as observed for the genera Pseudosinella, Trogolaphysa, Arrhopalites, and Pararrhopalites. Such 
cryptic diversity can only be accessed by specialized scrutiny or molecular sequencing. Cryptic diversity in Collembola cannot be explained by accelerated rates of molecular evolution [25], therefore, the diversity of subterranean Collembola in ferruginous MSS and caves, possibly results of the combination of the effects of lithology arrangement, phytophysiognomy and climate fluctuation at local scale.

Finally, the recognition of cryptic species within presumed widespread allopatric species is crucial to efficiently develop management and conservation plans [20] and reduce the underestimation of cave Collembola diversity.

\section{Subterranean Collembola Hot Spot}

Brazil presents a total of 60 species of cave Collembola recorded so far, 39 are records from the State of Minas Gerais, including 28 troglobites (Table 1). Iron caves and MSS respond for 26 of those troglobitic species and provide habitat to additional 12 troglophiles. This is even more remarkable considering that, most of collection and research on cave fauna is directed to larger caves, usually in limestone.

Two important subterranean hot spots in Limestone caves in the States of Sao Paulo and Bahia (Southeastern and Northeastern regions), presented an overall species richness of 28 and 22 troglobites respectively. The two caves are under different impact pressures, the former is in a protected area with controlled access, and the latter is under intense touristic exploitation [3].

Myers et al. [1] combined richness, endemism, distribution spam and threats to the area to define places of priority for conservation, called hot spots. For subterranean diversity, eventually, an arbitrary number of troglobites is used to define hot spots, disregarding threats to the conservation [2]. The number of troglobites, with a full consideration of the threats or conservation conditions of the caves and surroundings, was recently used as criteria for defining hotspot [3]. Another hot spot for subterranean fauna is recorded from Rio Grande do Norte, Northeastern Brazil, they assumed the same criteria for defining the caves systems in the study as an "oasis of subterranean biodiversity" [5], where the diversity and threats were fully addressed.

The ferruginous rock outcrops in Brazil are under a ferocious economic pressure, the mining industry represents an important part of the production of commodities as iron ore and steel. The metallogenic deposits in Minas Gerais represent $0.15 \%$ of the Brazilian territory, nearly $12,000 \mathrm{~km}^{2}$ [21], the elevated diversity of cave Collembola found in recent studies, 26 troglobites and 12 troglophiles in iron caves and MSS (Table 1), the high degree of endemicity, considering these species are found nowhere else the beta diversity is directly impacted, and the continuous threat to the subterranean habitats formed in ferruginous rock, justify categorizing the ferruginous subterranean habitats as hot spot for cave Collembola in the State of Minas Gerais. It is important to 
remark that the diversity considered here is only for Collembola species, and that the studies mentioned above have a much higher phylogenetic diversity, but it may be an effect of the lack of sampling for other taxa, and the phylogenetic diversity of ferruginous subterranean fauna may prove to be much higher when all the fauna is accessed.

\section{Conservation Policy Implications}

The ferruginous caves and the MSS represent sites of intense cryptic diversification, Katz et al. [20] observed that, for Collembola the detection of short-range endemics, genetic isolation, and apparent cryptic diversity has major conservation implications. The combination of morphological and molecular techniques together can recover the cryptic diversity of Collembola in a complementary way [24].

The results we present here bring several considerations on conservation strategies and policies. The high diversity and endemism rate observed for cave Collembola, associated to threats to the subterranean environments as mining, deforestation, and urbanization flag these areas as maximum priority and interest for planning putative conservation areas [26]. These areas demand a multi-factor approach to successfully develop policies which optimize the diversity conservation, particularly subterranean diversity.

Brazilian legislation has protective measures for caves, but allows the complete suppression of a cave for mining or other exploratory purpose, under a process for licensing the proposed activities. Even though some criteria are imposed, it fails in considering some important aspects of the cave structure in different lithology [9]. Here we observed that the whole process needs a revision when comes to ferruginous rock, where the cave may not be the important spatial unit to preserve, instead, the high subterranean diversity areas must be surveyed, not only in caves but also in the MSS. It is possible that in some cases to protect a hill that harbors a thick layer of Canga with a troglobitic species rich MSS, would result more effective to preserve restricted subterranean fauna, than to protect a small and shallow cave with reduced troglobitic richness.

The state of Minas Gerais has 75 integral conservation units (defined by law), with maximum protection policy, however, these conservation units represent only $1.05 \%$ ( $619,800 \mathrm{ha})$ of the state territory. There are other categories of conservation units, called of "sustainable use", with much less restrictive policies. These categories of conservation units are much less effective in order to preserve epigean species, due to the diverse usages and practices in those areas. Nevertheless, the 19 sustainable use conservation units in the state of Minas Gerais (private conservation units excluded) correspond to $3.01 \%(\sim 1,768,000 \mathrm{ha})$ of the state territory (ief.mg.gov.br/unidades-de-conservacao - accessed Sep/31/2021). Sustainable use conservation units have some criteria that prevents highly destructive activities, allowing some extractive crops, 
subsistence agriculture and tourism. These activities may be compatible with subterranean conservation through the MSS, therefore the conservation units network can get some advantage trying to connect integral conservation units with sustainable use ones. It was proposed that the sampling for subterranean fauna in prospection drilling holes all over the area may bring important information about species richness and distribution, mainly if combined with cave and surface sampling [27]. This procedure, implemented in the process for licensing new high impact exploratory activities, can improve the conservation effectiveness of the conservation units and compensation areas, precisely define the role of the cave in the conservation plan, and shift the focus towards troglobitic species richness.

\section{Conclusions}

Our results depict the ferruginous subterranean environment as an important hot spot for cave Collembola in the state of Minas Gerais, corroborating the expected high species richness in ferruginous rock caves and MSS. We also demonstrate that access cryptic diversity as observed in the genera Arrhopalites, Pararrhopalites, Pseudosinella and Trogolahysa is mandatory for planning the conservation strategies for subterranean Collembola. The distribution of the species through the MSS can be favored by sustainable use conservation units, whether this fauna is surveyed along the licensing process. Finally, we conclude that the conservation planning for future conservation unit establishment must focus not only on caves but also in the MSS, accessing the fauna through sampling in prospection drilling holes. Protecting an area with high richness of endemic troglobites down in the MSS may be more effective than to protect a shallow cave when it comes to preserve troglobitic diversity. 


\section{Methods}

Cryptic diversity. The richness was the measure of the subterranean diversity, we surveyed all data about previous records for Brazilian Collembola cave species, ecological status, lithology, and distribution from the literature, and included 11 newly found cryptic species from subterranean habitats in iron and limestone rock. The cryptic species were verified by comparison of chaetotaxy and "micro-morphology" through optic and scanning microscopy of disjunct populations of a widespread morphotype. The imagery was compared under hypotheses of chaetotaxic and morphologic homology, previously defined by different authors. Those populations with consistent discrete chaetotaxic and morphologic patterns were assumed to be independent species, therefore they were taxonomically diagnosed, named, and ordered in a dichotomic identification key with all Brazilian species of the genus, to test the validity of the species.

Microscopy. Specimens were preserved in ethanol 70\% and mounted on slides following Jordana et al. [28], after clearing using Nesbitt's solution for study under phase contrast microscope, line drawings were made with help of a drawing tube. For Scanning Electronic Microscope (SEM) study, specimens were dehydrated by ethanol, dried in a critical point dryer, and covered in gold.

Homology. The terminology used in the diagnoses for the hypotheses of homology followed: labial chaetotaxy after Gisin [29] with additions of Zhang \& Pan [30]; Fjellberg [31] for labial palp papillae and maxillary palp; postlabial chaetotaxy after Chen \& Christiansen [32], with adaptations of Cipola et al. [33] for $\mathbf{J}$ series; clypeal chaetotaxy after Yoshii \& Suhardjono [34]; labral chaetotaxy after Cipola et al [35]; unguiculus lamellae after Hüther [36]; Anterior dens chaetotaxy after Oliveira et al. [37]; Mari-Mutt [38] for dorsal head chaetotaxy, with additions of Soto-Adames [39]; Szeptycki [40] and Zhang \& Deharveng [41] for S-chaetotaxy; and Szeptycki [42] for dorsal chaetotaxy, with additions and modifications provided by Soto-Adames [39] and Zhang et al. [43]. Symbols used to depict the chaetotaxy are presented in Fig. 3 (A-C). Codes will be used in italics along the text to replace the morphological description of each chaeta and sensillum type. Additional information about morphology and chaetotaxy of discussed species was obtained from the literature.

Abbreviations used in the diagnoses. Ant-antennal segment(s); b.c.-basal chaeta(e), t.a.-terminal appendage of the maxillary palp; 1.p.-lateral process of labial papilla E, lpc-labial proximal chaeta(e); Th-thoracic segment; Abd-abdominal segment(s); Omt-trochanteral organ; a.e.-anteroexternal lamella, a.i.-antero-internal lamella, a.t.-unguis apical tooth, b.a.-basal anterior tooth of unguis, b.p.-basal posterior tooth of unguis, m.t.-unguis median tooth, p.i.-postero-internal lamella, p.e.-postero-external lamella; mac-macrochaeta(e), mes-mesochaeta(e), mic-microchaeta(e), msspecialized microchaeta(e), psp-pseudopore(s), sens-specialized ordinary chaeta(e) (sensillum), MSS-Mesovoid Shallow Substratum. 
Ecological status. To avoid subjectivity and ambiguity to determine the ecological status of the species, we assumed to be a troglobite all the species with some degree of troglomorphism exclusively distributed in the subterranean environment, either caves, MSS, or both. Species distributed in the surface and subterranean habitats were assumed to be troglophiles.

\section{Identification Key for the known and new species of the genus Trogolaphysa recorded in}

\section{Brazil.}

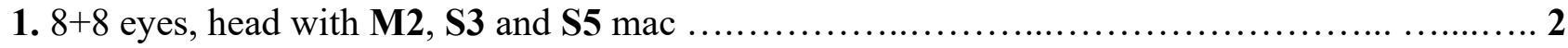

- 4+4 eyes or less, head with M2, S3 e S5 mic (Exc. T. dandarae sp. nov. S5 mac) ................ 5

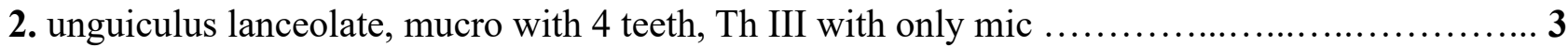

- unguiculus truncate, mucro with 5 teeth, Th III with $3+3$ mac ... T. formosensis (Baía formosa, RN)

3. unguiculus with pe lamela serrate, dens with two rows of spines

- unguiculus with pe lamela smooth, dens with only one row of spines .... T. hirtipes (Blumenau, SC)

4. anterior collophore with distal 4+4 mac, Abd IV with C4 and T7 mes, manubrial plate with 2 psp

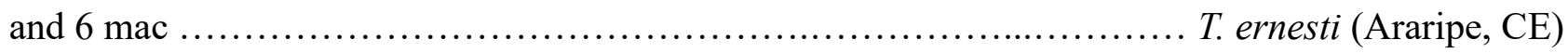

- anterior collophore with distal 3+3 mac, Abd IV com C4 e T7 mic, manubrial plate with 3 psp and $5 \mathrm{mac}$ T. piracurucaensis (Piracuruca, PI)

5. inner lamella of unguis with 1-2 unpaired teeth

- inner lamella of unguis without unpaired teeth 12

6. Th II p3 complex with $5+5$ or $6+6$ mac ........................................... 7

- Th II p3 complex with $4+4$ or $3+3$ mac ................................................... 10

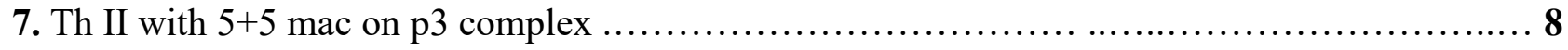

- Th II with 6+6 mac on p3 complex ............ T. lacerta sp. nov. (Conceição do Rio Acima, MG)

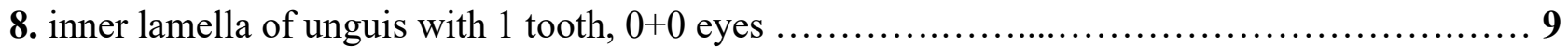

- inner lamella of unguis with 2 teeth, $0+0$ or $4+4$ eyes .......... T. zampauloi sp. nov. (Ribeira, SP)

9. unguiculus with p.e. lamella serrate, Abd IV with $3+3$ central mac (A3, B4-

5) T. gispertae sp. nov. (Parauapebas, PA)

- unguiculus com lamela p.e. lisa, 4+4 central (A3, A5, B4-5) mac on Abd IV T. crystallensis sp. nov. (Mariana, MG)

10. anterior collophore with distal $2+2$ mac, tenent hair acuminate 11 - anterior collophore distal 3+3 mac, tenent hair capitate ...T. bellinii sp. nov. (Barão de Cocais, MG) 11. Th II p3 complex with $4+4$ mac, unguiculus with p.e. lamella serrate, $4+4$ central $(\mathbf{A 3}, \mathbf{A 5}, \mathbf{B} 4-$ 5) mac on Abd IV T. chapelensis sp. nov. (Conceição do Rio Acima, MG) - Th II p3 complex with 3+3 mac, unguiculus with p.e. lamella smooth, Abd IV with 3+3 central mac (A3, B4-5) T. epitychia sp. nov. (Conceição do Mato Dentro, MG)

12. external row of spines on dens with more than 30 spines 
- external row of spines on dens with less than 20 spines T. hauseri (Gruta da tapagem, SP)

13. Th II p3 complex with $5+5$ or $6+6$ mac

- Th II p3 complex with 3+3 T. mariecurieae sp. nov. (Conceição do Mato Dentro, MG)

14. Mucro with 4 teeth, tenent hair acuminate 15

- Mucro with 3 teeth, tenent hair capitate T. dandarae sp. nov. (Parauapebas, PA)

15. unguiculus lanceolate, dens with two rows of spines 16 - unguiculus truncate, dens with one row of spines T. tijucana (Três Rios, RJ)

16. Abd IV with $2+2$ central mac (B4-5), anterior collophore with distal $2+2$ mac T. sotoadamesi sp. nov. (Mariana, MG)

- Abd IV with 3+3 central mac (A3, B4-5), anterior collophore with distal 3+3 $\operatorname{mac}$ T. barroca sp. nov. (Mariana, MG)

* T. aelleni and T. millsi are omitted due to insufficient description of their morphology and chaetotaxic characters.

Taxonomic diagnoses and morphological plates. Type materials are deposited in the Coleção de Referência de Fauna de Solo, Universidade Estadual da Paraíba (CRFS-UEPB) and Museu Nacional Rio de Janeiro, Universidade Federal do Rio de Janeiro (MNRJ-UFRJ). zoobank.org:pub:2C8F4446-0869-48A9-ABD7-14D4C4DCB0FA

Additional records in Supplementary Material S1, taxonomic references in S2.

Family Paronellidae Börner, 1906

Subfamily Paronellinae Börner, 1906

Tribe Paronellini sensu Zhang et al., 2019

Genus Trogolaphysa Mills, 1938

(Figures 2-10)

Diagnosis. Habitus typical of this genus (Figs. 2A-D), hyaline scales presents on Ant. I-II, head, body, and ventral face of furcula (Figs. 2C-D; Figs. 3A-C; Fig. 4D, 4F; Figs. 6-7; Fig. 10C), Ant IV smooth or annulated and never subdivided in two (Fig. 4A); eyes 0-8 (ex. Fig. 5C); prelabral and labral formula 4/5,5,4 (prelabral smooth or ciliate, pma smooth chaetae) (Fig. 5A); antennobasal-organ present (Fig. 5C); labial chaetae L1-2 not reduced (Fig. 5E); sublobal plate of maxillary palp with 2 chaetae (Fig. 5E); Th II normally with $\mathbf{a 5}$ mac and $\mathbf{p 3}$ complex with variable number of mac, and Th III with $\mathbf{p} 3$ mac present or abset (Fig. 6A-B), abdominal segments II-IV with 2, 3, 3 bothriotricha (Figs. 6C-D; Fig. 7A); unguis with three external lamellae and unguiculus with p.e. lamella serrate or smooth (Fig. 8A-C); trocanteral organ with 2-4 psp (Fig. 9A) collophore anterior side with 2-3 distal mac (Fig. 9C); tenaculum with four teeth on each branch and one anterior chaeta (Fig. 9D); manubrium without spines, manubrial plate with 2-3 psp (Fig. 
10A); anterior proximal dens with b.a., b.m. and i5 chaetae (Fig. 10B); dens with 1-2 rows of spines; mucro square or rectangular but relatively short, with 3-5 teeth (Fig. 10D). 

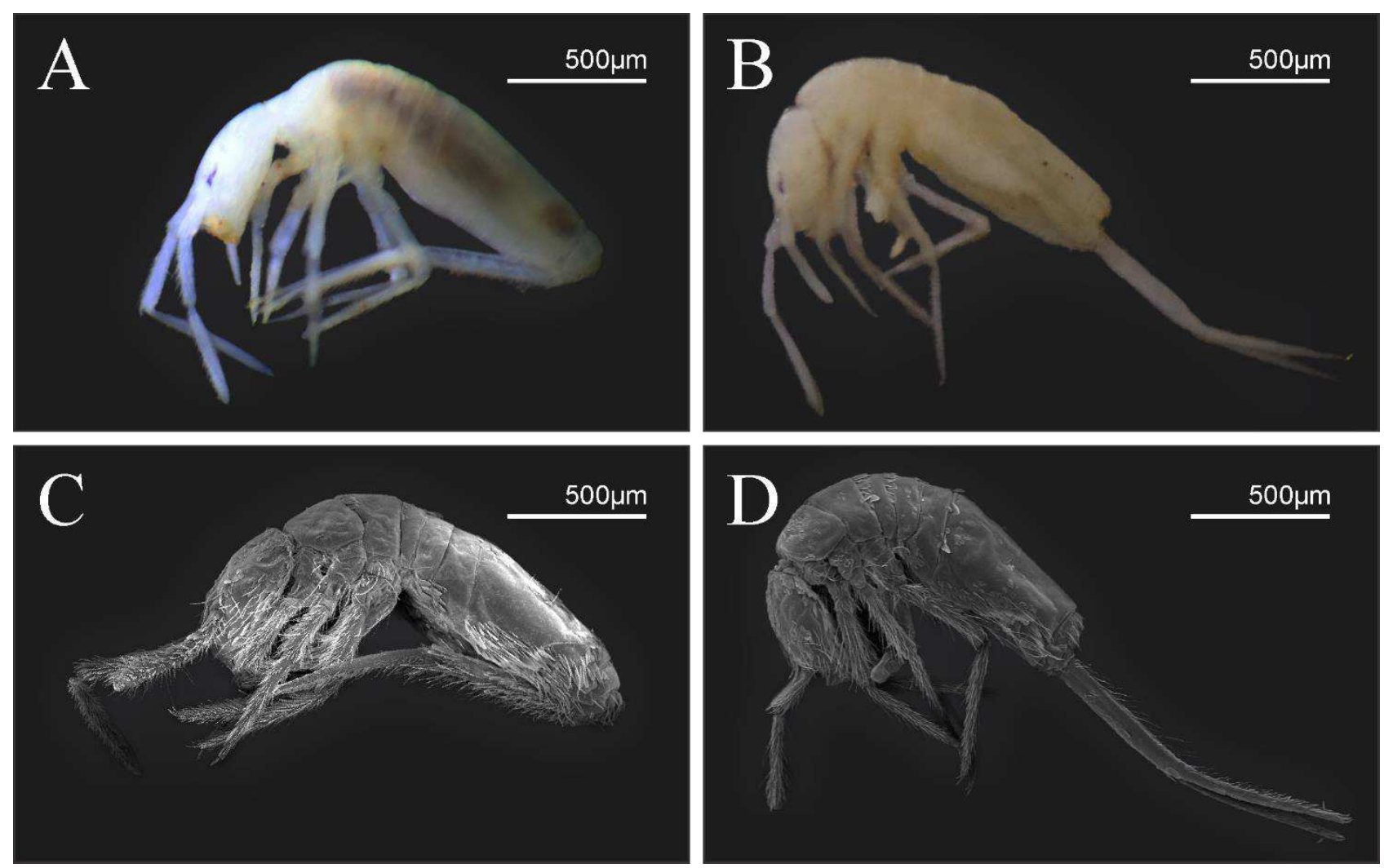

Figure 2. Trogolaphysa sp.: habitus lateral view. A-B) specimen fixed in ethanol; C-D) SEM photographs. 

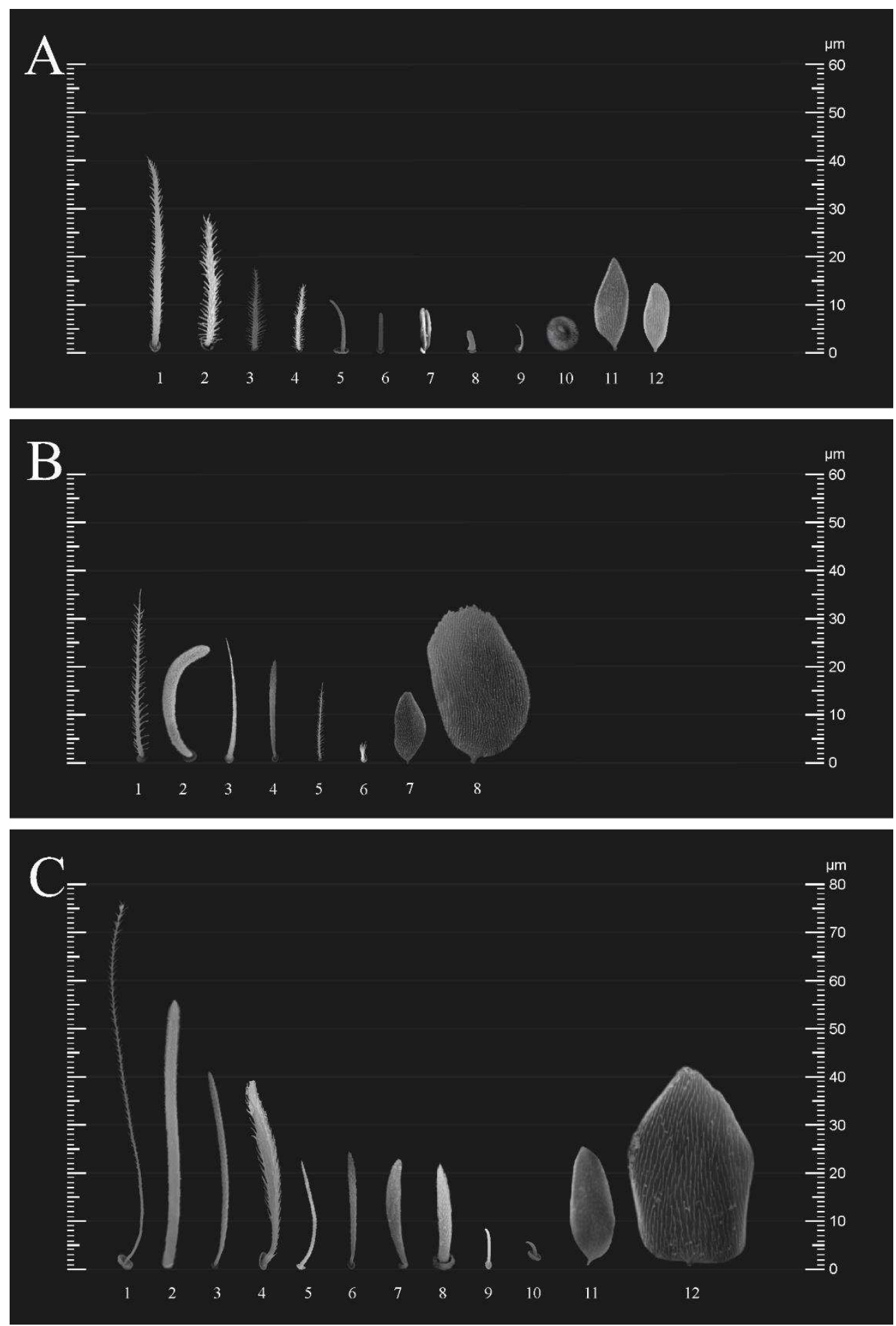

Figure 3. Trogolaphysa sp. SEM: general body chaetae. A) Antennal chaetae, sensilla and scales: 1macrochaeta with short ciliation, 2- macrochaeta with long ciliation, 3- microchaeta with long ciliation, 4- microchaeta with short ciliation, 5- finger-shaped sens, 6- wrinkly sens, 7- coffee bean shaped sens, 8- rod sens, 9- spine-like sens, 10- Ant IV subapical-organ, 11- lanceolate scale, 12rounded scales; B) Head chaetae and scales: 1- strait macrochaeta with long ciliation, 2- blunt macrochaeta, 3- smooth chaeta, 4- blunt chaeta, 5- strait microchaeta with long ciliation, 6- labial r microchaeta, 7- cephalic anterior scale, 8- cephalic posterior scale; C) Body and appendages chaetae, sens and scales: 1- bothriotrichum, 2- blunt macrochaeta, 3- blunt mesochaeta, 4- dens external ciliate chaeta, 5- smooth microchaeta, 6- blunt microchaeta, 7- fan-shape chaeta, 8-dental spine, 9- 'al' sens, 10- 'ms' sens, 11- lanceolate scale, 12- intersegmental scale. 


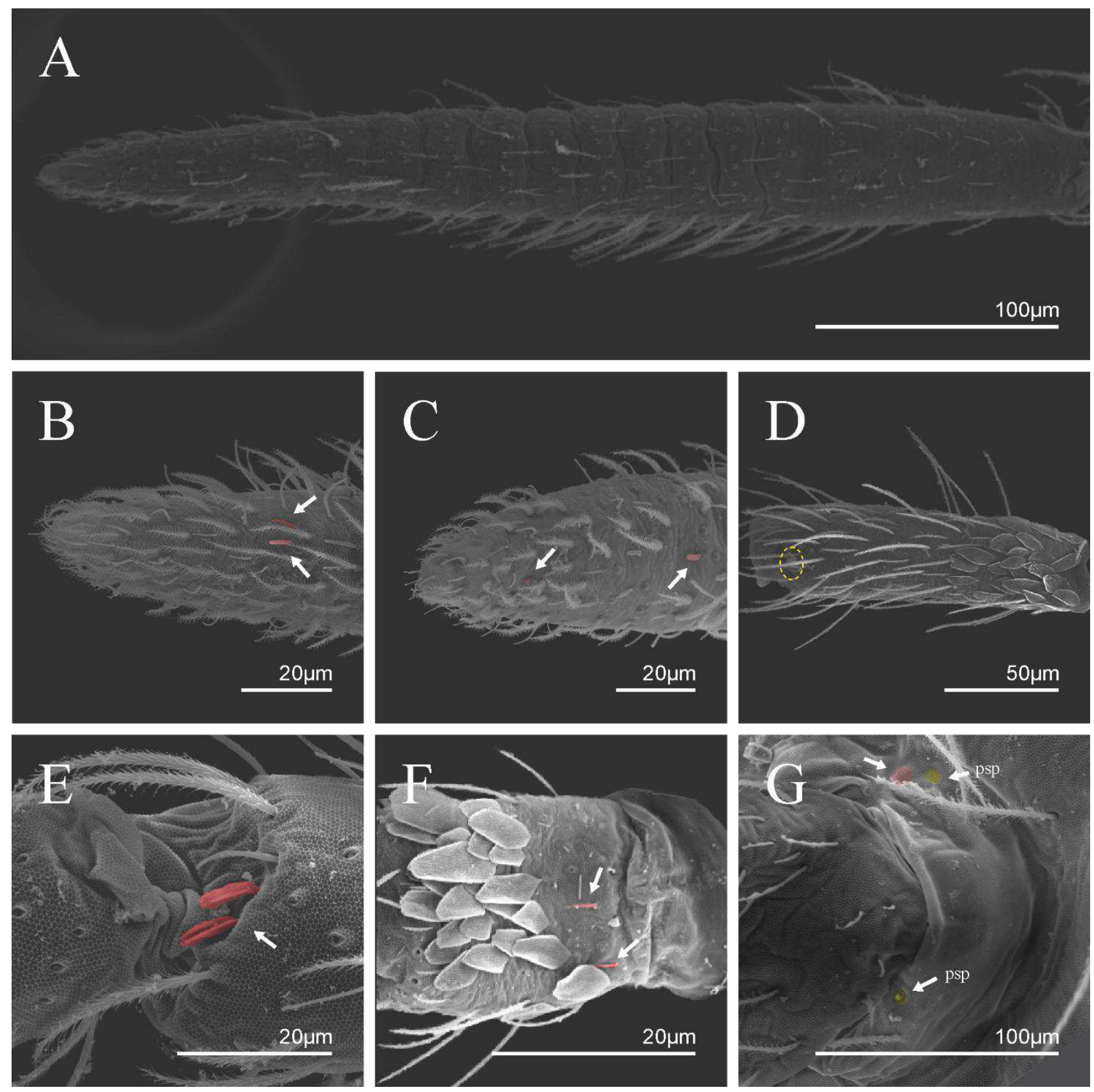

Figure 4. Trogolaphysa sp. SEM: antenna: A) Ant IV dorsal view; B) Ant IV apex dorsal view, arrow indicates finger-shaped and wrinkly sens; C) Ant IV apex ventral view, left arrow indicates Ant IV subapical-organ, right arrow point one sensillum type A8; D) Ant II dorsal view, dashed line indicates rod sens; E) Detail of the sensilla of the Ant III apical organ (red); F) Ant I dorsal view spine like sens (arrows indicate the sensilla in red); G) detail of the Ant I basal, arrow indicates psp and antenobasal organ (yellow and red respectively). 

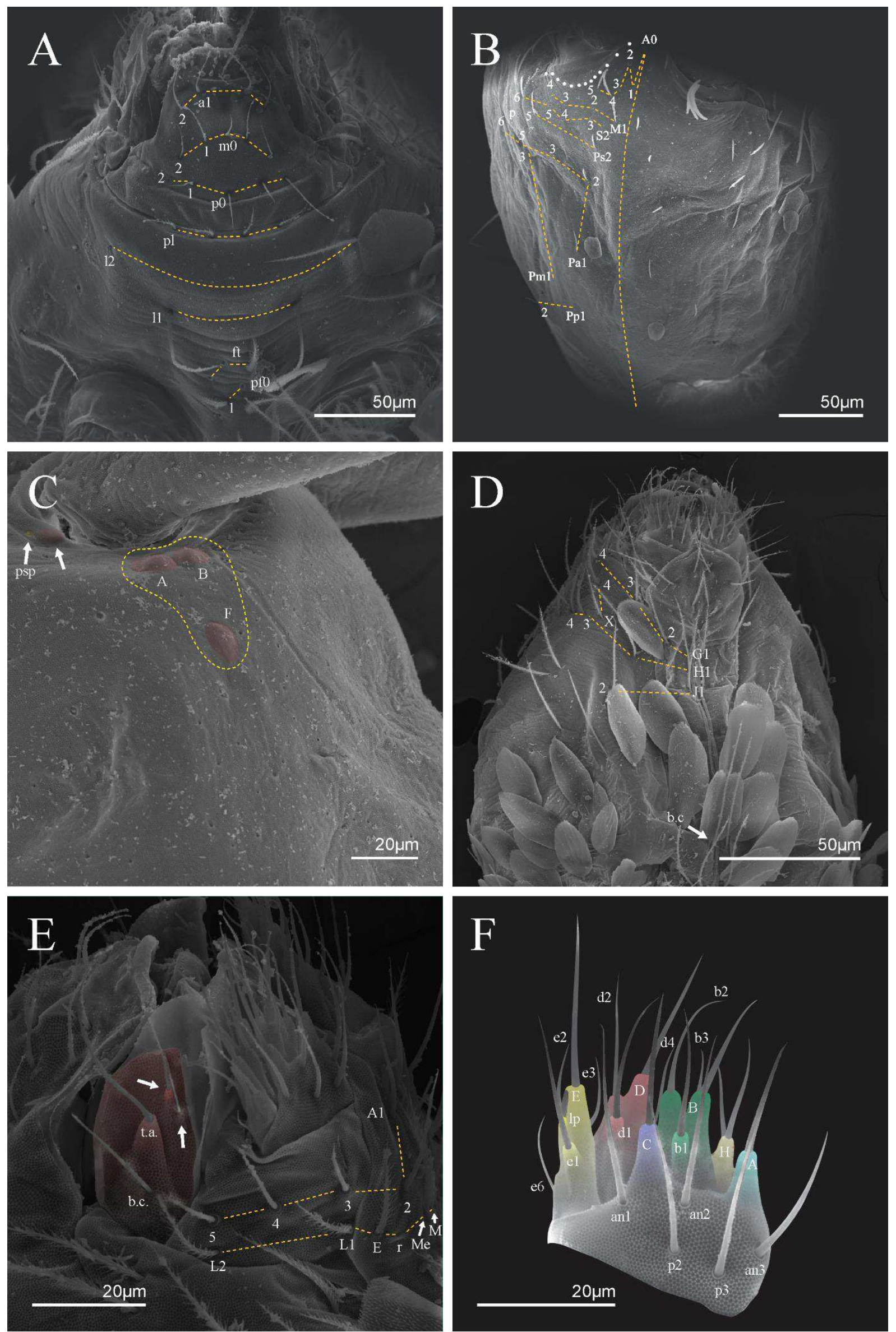

Figure 5. Trogolaphysa sp. SEM: head and mouthpart chaetotaxy. A) clypeus; B) dorsal head; C) eyes (red) circled by dashed line, arrow indicates antenobasal organ and psp; D) ventral head; E) maxillary palp and sublobal plate (right side); F) detail of maxillary palp. 

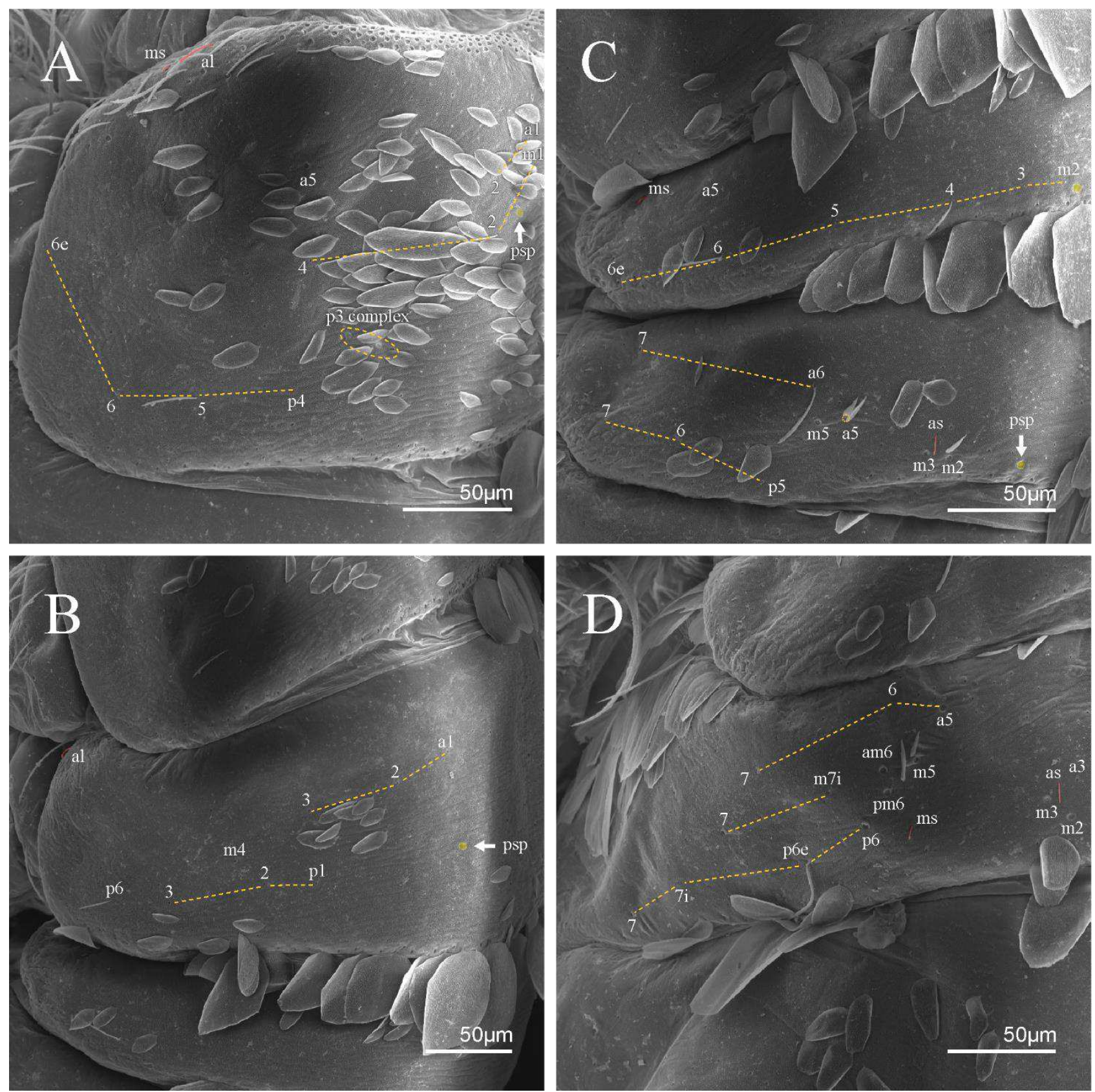

Figure 6. Trogolaphysa sp. SEM: thorax and abdomen dorsal chaetotaxy: A) Th II; B) Th III, C) Abd I-II, D) Abd III. 

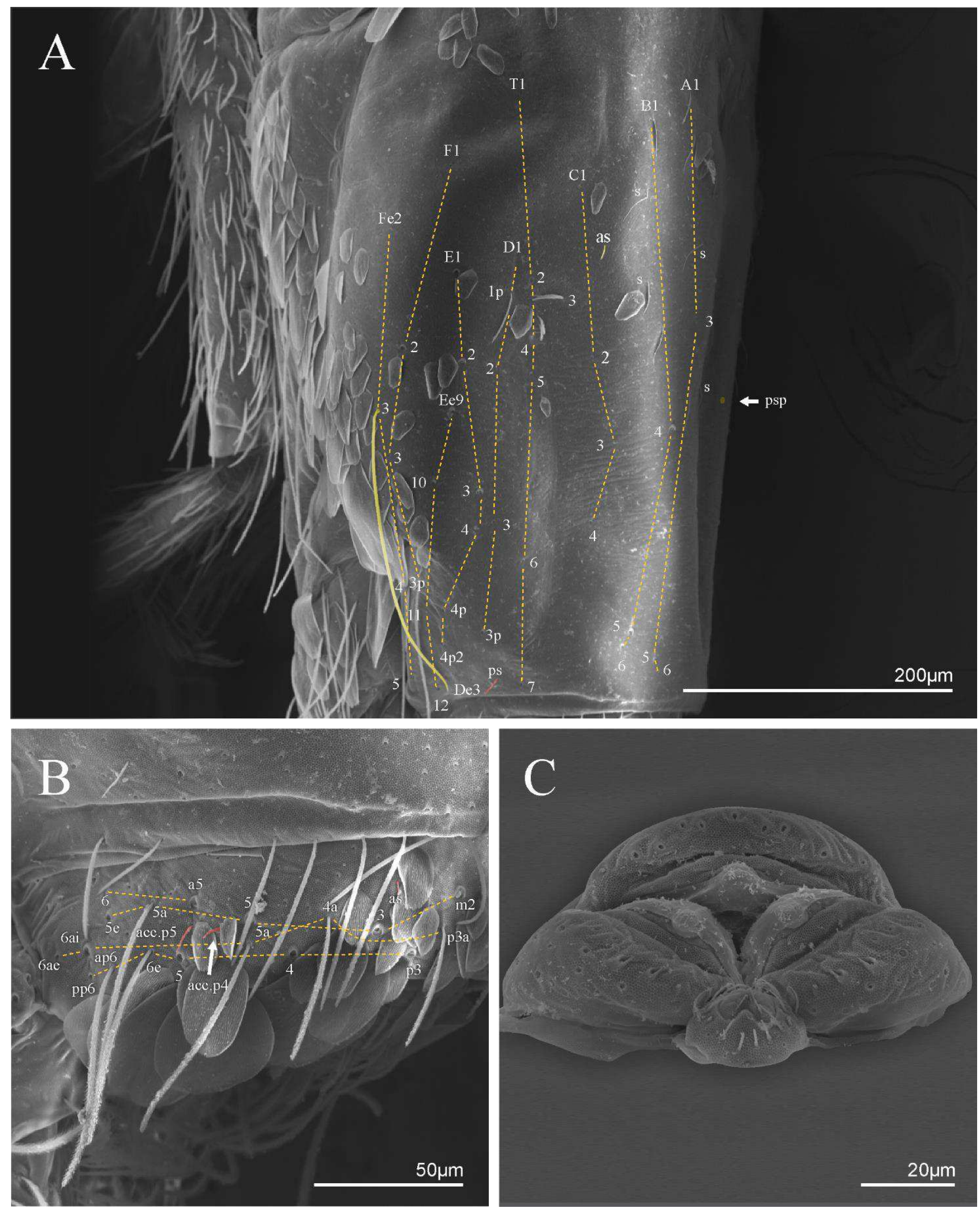

Figure 7. Trogolaphysa sp. SEM: A) Abd IV dorsal chaetotaxy; B) Abd V dorsal chaetotaxy; C) anal pore and male genital papilla. 

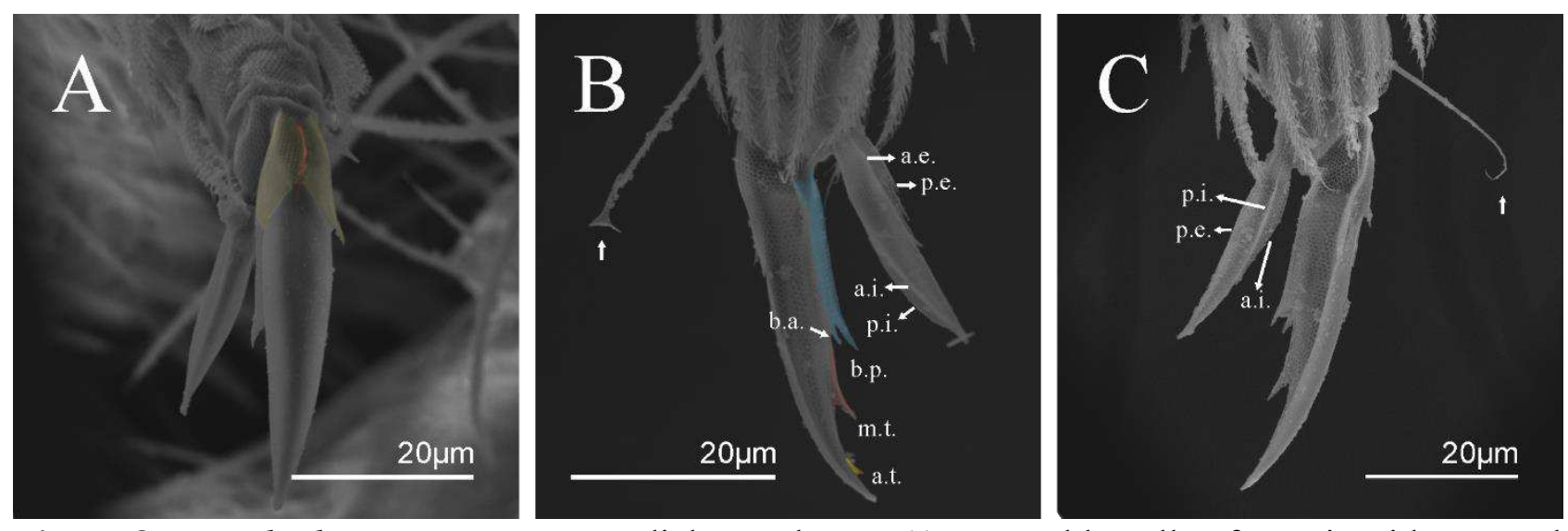

Figure 8. Trogolaphysa. sp. SEM: empodial complex III A) external lamella of unguis with external teeth (pseudonychia, yellow); B) unguis and unguiculus lateral view, unguis internal lamella with basal, medial and apical teeth (blue, red and yellow respectively), unguiculus with internal and external teeth, tenent hair capitate (white arrow); C) lateral view, unguiculus lamellae, tenent hair acuminate (white arrow). 

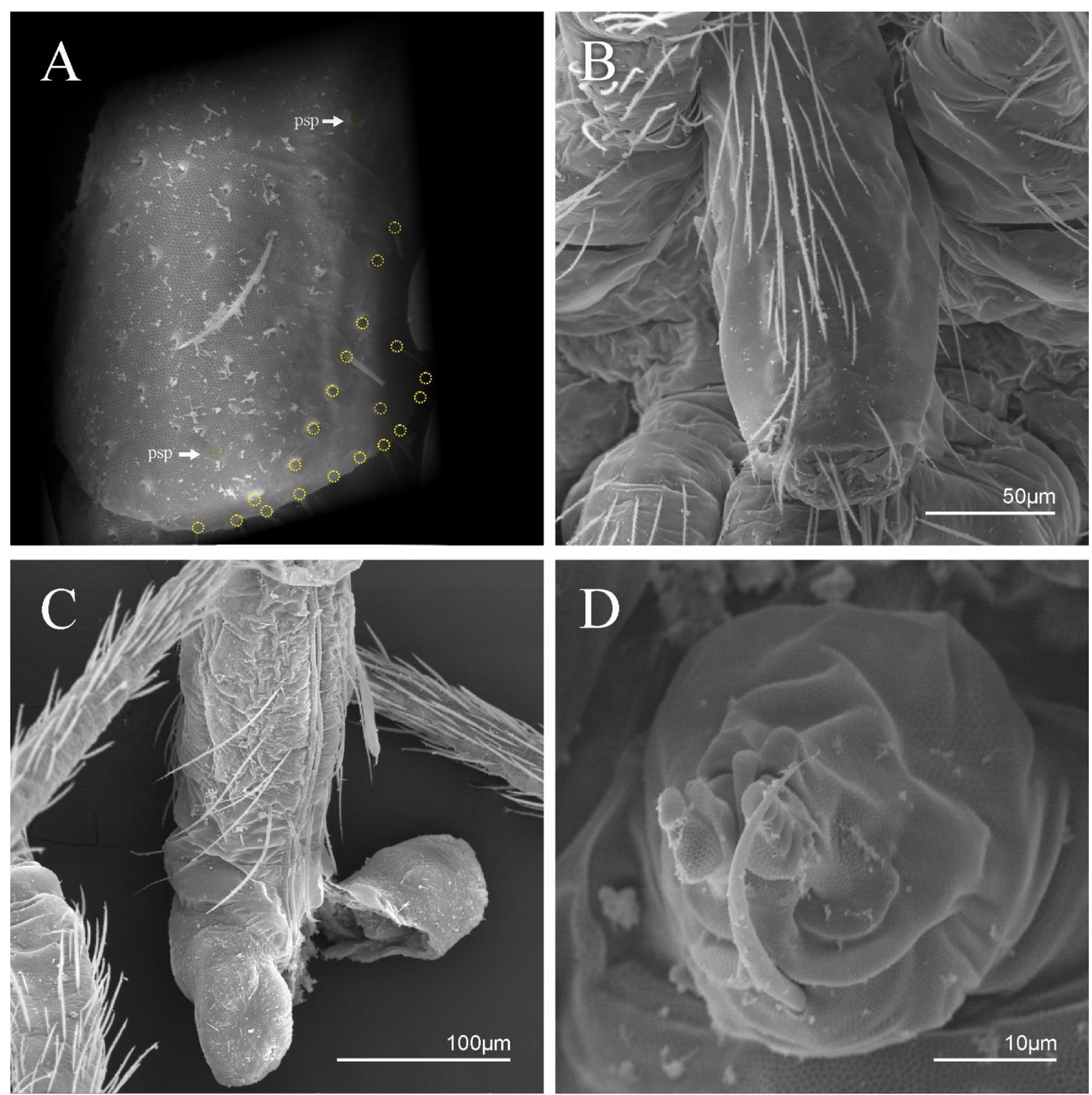

Figure 9. Trogolaphysa sp. SEM: appendages A) Metatrochanteral organ with pseudopores (alveoli marked in yellow, white arrows indicate pseudopores); B) ventral tube posterior chaetae; C) ventral tube anterior chaetae; D) Tenaculum. 

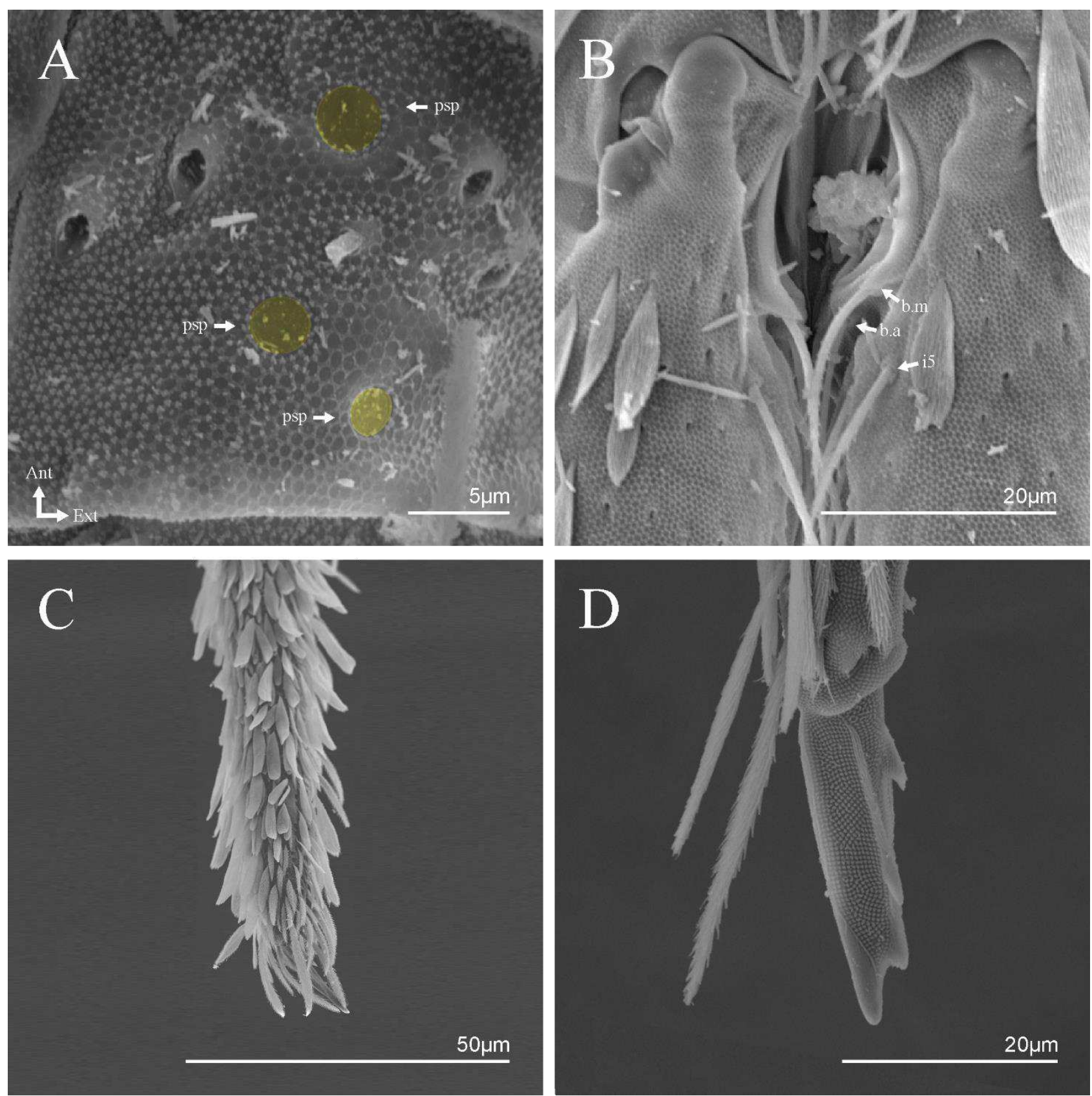

Figure 10. Trogolaphysa sp. SEM: furca. A) manubrial plate pseudopores (yellow); B) anteroapical chaetae of dens; C) dens anterior view; D) mucro. 


\begin{tabular}{|c|c|c|c|c|c|c|c|c|c|c|c|c|c|c|}
\hline & 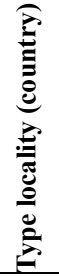 & 䒿 & ఫે & 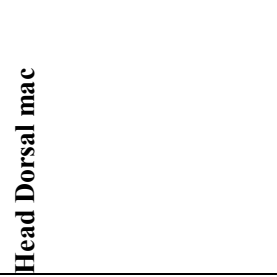 & 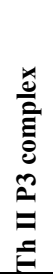 & $\underset{\Xi}{\stackrel{E}{\Xi}}$ & $\begin{array}{l}\underset{\Xi}{\Xi} \\
己 \\
Z \\
Z\end{array}$ & $\sum_{2}^{\frac{0}{2}}$ & 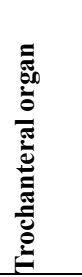 & 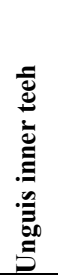 & 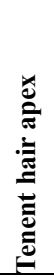 & 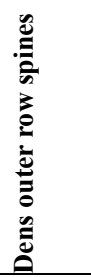 & 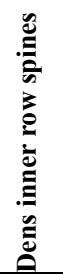 & $\stackrel{巳}{\stackrel{E}{E}}$ \\
\hline T. aelleni Yoshii, 1988 & $\mathrm{Br}$ & $\mathrm{CV}$ & 2 & $?$ & $?$ & $?$ & $?$ & $?$ & 18 & 3 & $\mathrm{~A}$ & $?$ & $?$ & 4 \\
\hline T. barroca sp. Nov. & $\mathrm{Br}$ & $\mathrm{Cv}$ & 0 & $\mathrm{~A} 0, \mathrm{~A} 2, \mathrm{~Pa} 5$ & 5 & 0 & $\begin{array}{l}\text { A3, B4- } \\
5\end{array}$ & 4 & $16-21$ & 2 & A & $37-39$ & $\begin{array}{l}21- \\
22\end{array}$ & 4 \\
\hline $\begin{array}{l}\text { T. belizeana Palacios- } \\
\text { Vargas \& Thibaud } 1997\end{array}$ & $\mathrm{Be}$ & $\mathrm{Cv}$ & 0 & $\begin{array}{l}\mathrm{A} 0, \mathrm{~A} 2-3, \mathrm{M} 3, \mathrm{~S} 3, \mathrm{~S} 5 \\
\mathrm{~Pa} 5, \mathrm{Pm} 3\end{array}$ & 2 & 3 & $\begin{array}{l}\text { A4-5, } \\
\text { B5 }\end{array}$ & $4^{?}$ & 18 & 2 & A & 30 & 31 & 3 \\
\hline T. bellinii sp. Nov. & $\mathrm{Br}$ & $\mathrm{Cv}$ & $0-2$ & $\mathrm{~A} 0, \mathrm{~A} 2$ & 3 & 0 & $\begin{array}{l}\text { A3, A5, } \\
\text { B4-5 }\end{array}$ & 4 & 20 & $3-4$ & $\mathrm{C}$ & 24 & 25 & 4 \\
\hline $\begin{array}{l}\text { T. bessoni Thibaud \& } \\
\text { Najt } 1988\end{array}$ & $\mathrm{Ec}$ & $\mathrm{Cv}$ & 0 & $?$ & 2 & 0 & $\begin{array}{l}\text { A5, B4, } \\
\text { B5 }\end{array}$ & $?$ & 19 & 2 & A & 25 & 20 & $\begin{array}{l}4- \\
5\end{array}$ \\
\hline T. chapelensis sp. Nov. & $\mathrm{Br}$ & $\mathrm{Cv}$ & 0 & $\mathrm{~A} 0, \mathrm{~A} 2$ & 4 & 0 & $\begin{array}{l}\text { A3, A5, } \\
\text { B4-5 }\end{array}$ & 9 & 23 & $3-4$ & A & $>70$ & 30 & 4 \\
\hline $\begin{array}{l}\text { T. caripensis (Gruia, } \\
\text { 1987) }\end{array}$ & $\mathrm{Ve}$ & $\mathrm{Cv}$ & 0 & $\begin{array}{l}\text { A0, A2-3, M1-2, S2-3, } \\
\text { S5, Pa5, Pm3 }\end{array}$ & 6 & 0 & $\begin{array}{l}\text { A3, A5, } \\
\text { B4-5 }\end{array}$ & $?$ & 21 & 3 & A & $30-25$ & $\begin{array}{l}30- \\
25\end{array}$ & 4 \\
\hline T. crystallensis sp. Nov. & $\mathrm{Br}$ & $\mathrm{Cv}$ & 0 & $\mathrm{~A} 0, \mathrm{~A} 2$ & 5 & 0 & $\begin{array}{l}\text { A3, A5, } \\
\text { B4-5 }\end{array}$ & 3 & 18 & 3 & A & 58 & 28 & 4 \\
\hline T. dandarae sp. Nov. & $\mathrm{Br}$ & $\mathrm{Cv}$ & 0 & $\mathrm{~A} 0, \mathrm{~A} 2, \mathrm{~S} 5, \mathrm{~Pa} 5, \mathrm{Pm} 3$ & 6 & 3 & $\begin{array}{l}\text { A3, B4- } \\
5\end{array}$ & 3 & 19 & 2 & $\mathrm{C}$ & $31-39$ & $\begin{array}{l}18- \\
21\end{array}$ & 3 \\
\hline $\begin{array}{l}\text { T. ecuatorica (Palacios- } \\
\text { Vargas, Ojeda \& } \\
\text { Christiansen, 1985) }\end{array}$ & $\mathrm{Ec}$ & $\mathrm{Cv}$ & 0 & $?$ & $?$ & $?$ & $?$ & $?$ & 2 & 2 & A & 45 & 45 & 5 \\
\hline T. epitychia sp. nov. & $\mathrm{Br}$ & $\mathrm{Cv}$ & 0 & $\mathrm{~A} 0, \mathrm{~A} 2$ & 3 & 0 & $\begin{array}{l}\text { A3, A5, } \\
\text { B5 }\end{array}$ & 3 & 15 & 3 & A & 60 & 34 & 4 \\
\hline $\begin{array}{l}\text { T. ernesti Cipola \& } \\
\text { Bellini, } 2017\end{array}$ & $\mathrm{Br}$ & $\mathrm{Lt}$ & 8 & $\mathrm{~A} 0, \mathrm{~A} 2-3$ & 6 & 0 & $\begin{array}{l}\text { A } 3, \text { A5, } \\
\text { B4-5 }\end{array}$ & $?$ & 49 & 4 & $\mathrm{C}$ & $21-27$ & $\begin{array}{l}23- \\
30\end{array}$ & 4 \\
\hline $\begin{array}{l}\text { T. formosensis Silva \& } \\
\text { Bellini, } 2015\end{array}$ & $\mathrm{Br}$ & $\mathrm{Lt}$ & 8 & $\begin{array}{l}\text { A0, A2, M2, S3, S5, } \\
\text { Pa5 }\end{array}$ & 6 & 3 & $?$ & 3 & 12 & 4 & $\mathrm{C}$ & $?$ & $?$ & 5 \\
\hline T. gisbertae sp. nov. & $\mathrm{Br}$ & $\mathrm{Cv}$ & 0 & $\mathrm{~A} 0, \mathrm{~A} 2-3, \mathrm{~Pa} 5, \mathrm{Pm} 3$ & 5 & 0 & $\begin{array}{l}\text { A3, B4- } \\
5\end{array}$ & 1 & 25 & 3 & A & 38 & $\begin{array}{l}21- \\
24\end{array}$ & 4 \\
\hline $\begin{array}{l}\text { T. haitica (Palacios- } \\
\text { Vargas, Ojeda \& } \\
\text { Christiansen, 1985) }\end{array}$ & $\mathrm{Ha}$ & $\mathrm{Cv}$ & 0 & $?$ & $?$ & $?$ & $?$ & $?$ & 22 & 2 & A & $30-38$ & $\begin{array}{l}30- \\
38\end{array}$ & 4 \\
\hline T. hauseri Yoshii, 1988 & $\mathrm{Br}$ & $\mathrm{Cv}^{?}$ & 0 & $?$ & $?$ & $?$ & $?$ & $?$ & 15 & 2 & A & 14 & 30 & 4 \\
\hline $\begin{array}{l}\text { T. hirtipes (Handschin, } \\
\text { 1924) }\end{array}$ & $\mathrm{Br}$ & $\mathrm{Tp}$ & $8^{?}$ & $?$ & $?$ & $?$ & $?$ & $?$ & $?$ & $4^{?}$ & $\mathrm{C}$ & $?$ & $?$ & 4 \\
\hline $\begin{array}{l}\text { T. hondurensis } \\
\text { (Palacios-Vargas, Ojeda } \\
\text { \& Christiansen, 1985) }\end{array}$ & Ho & $\mathrm{Cv}$ & 4 & $?$ & $?$ & $?$ & $?$ & $?$ & $?$ & 2 & A & $30-36$ & $\begin{array}{l}30- \\
36\end{array}$ & 4 \\
\hline $\begin{array}{l}\text { T. jacobyi Soto-Adames } \\
\text { \& Taylor } 2013\end{array}$ & $\mathrm{Be}$ & $\mathrm{Cv}$ & 0 & $\begin{array}{l}\text { A0, A2-3, M2, S3, } \\
\text { S5 ,Pa5, Pm3 }\end{array}$ & 2 & 1 & $\begin{array}{l}\text { A5, B4, } \\
\text { B5 }\end{array}$ & $?$ & 25 & 3 & A & $?$ & 36 & 3 \\
\hline T. lacerta sp. nov. & $\mathrm{Br}$ & $\mathrm{Cv}$ & $0-3$ & $\mathrm{~A} 0, \mathrm{~A} 2$ & 6 & 0 & $\begin{array}{l}\text { A3, B4- } \\
5\end{array}$ & 5 & 24 & 4 & A & 50 & 37 & 4 \\
\hline T. mariecurieae sp. nov. & $\mathrm{Br}$ & Ms & 0 & $\mathrm{~A} 0, \mathrm{~A} 2$ & 3 & 0 & A4 B5 & 4 & 15 & 3 & $\mathrm{C}$ & 40 & 22 & 4 \\
\hline $\begin{array}{l}\text { T. marimutti (Palacios- } \\
\text { Vargas, Ojeda \& } \\
\text { Christiansen, 1985) }\end{array}$ & $\mathrm{Me}$ & $\mathrm{Cv}$ & 0 & $?$ & $?$ & $?$ & $?$ & $?$ & 15 & 3 & A & 50 & 50 & 4 \\
\hline T. millsi Arlé, 1939 & $\mathrm{Br}$ & $\mathrm{Lt}$ & 2 & $?$ & $?$ & $?$ & $?$ & $?$ & $?$ & 3 & $\mathrm{~A}^{?}$ & $?$ & $?$ & 4 \\
\hline $\begin{array}{l}\text { T. oztotlica (Ojeda \& } \\
\text { Palacios-Vargas, 1984) }\end{array}$ & $\mathrm{Me}$ & $\mathrm{Cv}$ & 0 & $\mathrm{~Pa} 5, \mathrm{Pm} 3 ?$ & $?$ & $?$ & $?$ & $?$ & 14 & 4 & A & $35-40$ & $\begin{array}{l}35- \\
40\end{array}$ & 4 \\
\hline $\begin{array}{l}\text { T. piracurucaensis } \\
\text { Nunes \& Bellini, } 2018\end{array}$ & $\mathrm{Br}$ & $\mathrm{Lt}$ & 8 & $\begin{array}{l}\mathrm{A} 0, \mathrm{~A} 2-3, \mathrm{M} 2, \mathrm{~S} 3, \mathrm{~S} 5 \\
\mathrm{~Pa} 5, \mathrm{Pm} 3\end{array}$ & 6 & 0 & $\begin{array}{l}\text { A3, A5, } \\
\text { B4-5 }\end{array}$ & $?$ & $35-40$ & 4 & $\mathrm{C}$ & $21-27$ & $\begin{array}{l}25- \\
29\end{array}$ & 4 \\
\hline T. sotoadamesi sp. nov. & $\mathrm{Br}$ & $\mathrm{Cv}$ & 0 & $\mathrm{~A} 0, \mathrm{~A} 2$ & 5 & 0 & B4-5 & 4 & $19-21$ & 2 & A & 35 & $\begin{array}{l}21- \\
26\end{array}$ & 4 \\
\hline $\begin{array}{l}\text { T. tijucana (Arlé \& } \\
\text { Guimarães, 1979) }\end{array}$ & $\mathrm{Br}$ & $\mathrm{Lt}$ & 2 & $?$ & $?$ & $?$ & $?$ & $?$ & $?$ & 2 & $\mathrm{~A} ?$ & $?$ & $?$ & 4 \\
\hline $\begin{array}{l}\text { T. trioculata Soto- } \\
\text { Adames, } 2015\end{array}$ & $\mathrm{Me}$ & $\mathrm{Lt}$ & 3 & $\mathrm{~A} 0, \mathrm{~A} 2, \mathrm{~Pa} 5, \mathrm{Pm} 3$ & 5 & 0 & $\begin{array}{l}\text { A3, A5, } \\
\text { B5 }\end{array}$ & 4 & 14 & 3 & A & 12 & 15 & 4 \\
\hline $\begin{array}{l}\text { T. xtolokensis (Palacios- } \\
\text { Vargas, Ojeda \& } \\
\text { Christiansen, 1985) }\end{array}$ & $\mathrm{Me}$ & $\mathrm{Cv}$ & 0 & $\mathrm{~A} 0, \mathrm{~A} 2, \mathrm{~Pa} 5^{?}$ & $?$ & $?$ & $?$ & $?$ & 20 & 4 & A & $41-69$ & $\begin{array}{l}41- \\
69\end{array}$ & 4 \\
\hline T. zampauloi sp. nov. & $\mathrm{Br}$ & $\mathrm{Cv}$ & $0-4$ & $\mathrm{~A} 0, \mathrm{~A} 2$ & 5 & 0 & $\begin{array}{l}\text { A3, A5, } \\
\text { B4-5 }\end{array}$ & 3 & 27 & 4 & A & 30 & 23 & 4 \\
\hline
\end{tabular}

Table 2. Trogolaphysa species of the Neotropical Region, comparative morphology. Be, Belize; Br, Brazil; Ec, Ecuador; Ha, Haiti; Ho, Honduras; Me, Mexico. Cv, cave; Lt, Leaflitter, Tp, Termitophile, Ms, Mesovoid shallow substratum. ?, lacking or dubious information. 
Trogolaphysa bellinii sp. nov. Oliveira, Lima \& Zeppelini

Figures 11-13, Tables 1-2

Type material. Holotype female in slide (15482/CRFS-UEPB): Brazil, Minas Gerais State, Barão de Cocais municipality, cave MDIR-0028, next to “Mina de Brucutu”, 1952'48.7”S, 43²6'13.6”'W, 19-23.viii.2019, Carste team coll. Paratypes in slides (15468, 15483/CRFS-UEPB): 2 females, same data as holotype. Paratypes in slides (15519, 15576/CRFS-UEPB donated to MNJR): 2 females, same data as holotype. Additional records see S1.

Description. Total length (head + trunk) of specimens $1.53-1.75 \mathrm{~mm}(\mathrm{n}=5)$, holotype $1.70 \mathrm{~mm}$. Head. Ratio antennae: trunk $=1: 1.29-1.95(\mathrm{n}=5)$, holotype $=1: 1.95$; Ant III shorter than Ant II; Ant segments ratio as I: II, III, IV = 1: 1.80-2.24, 0.85-2.08, 0.85-2.08, holotype $=1: 1.80,0.85$, 1.34. Antennal chaetotaxy: Ant IV dorsally and ventrally with several short ciliate mic and mac, and finger-shaped sens, dorsally with a longitudinal row with about eight rod sens, ventrally with one subapical-organ and several wrinkly sens (Fig. 3A); Ant III dorsally and ventrally with several short ciliate mic and mac, and finger-shaped sens, dorsally without modified sens, ventrally with one apical psp, about three wrinkly sens on external longitudinal row, apical organ with two mic smooth chaetae externally, two coffee bean-like sens, and one rod sens (Fig. 3A); Ant II dorsally and ventrally with several short ciliate mic and mac, dorsally with four sub-apical finger-shaped sens, one wrinkly sens and two subapical rod sens, ventrally with one apical psp, about six wrinkly sens on longitudinal external row (Fig. 3A); and Ant I dorsally and ventrally with several short ciliate mic and mac, dorsally with three basal spine-like sens, ventrally with four basal spine-like sens, about five smooth mic and several finger-shaped sens (Fig. 3A). Eyes 0+0, rarely 2+2. Head dorsal chaetotaxy (Fig. 11A) with 12 An (An1a-3), six A (A0-5), five M (M1-5), five S (S2-6), two Ps (Ps2, Ps5), four Pa (Pa1-5), two Pm (Pm1, Pm3), seven Pp (Pp1-7), and two Pe (Pe4, Pe6) chaetae; Pa5 and Pm3 as mes, An1a-3a with 10 mac plus two mes, A0 and A2 as mac; interocular $\mathbf{p}$ mes present. Basomedian and basolateral labial fields with a1-5 smooth, M, Me, E and L1-2 ciliate, $\mathbf{r}$ reduced (Fig. 11B). Ventral chaetotaxy with 35-38 ciliate chaetae and one reduced lateral spine; postlabial G1-4; X, X4; H1-4; J1-2, chaetae b.c. present and a collar row of four to seven mes chaetae distally (Fig. 11B). Prelabral chaetae ciliate. Labral chaetae smooth, no modifications. Labial papilla $\mathbf{E}$ with l.p. finger-shaped and surpassing the base of apical appendage. Labial proximal chaetae smooth (an1-3, p2-3) and subequal in length (Fig. 11B). Maxillary palp with t.a. smooth and $1.23 \times$ larger than b.c.

Thorax dorsal chaetotaxy (Fig. 12A). Th II a, m, p series with two mic (a1-2), one mac (a5), three mic (m1-2, m4) and four mic (p4-6e), p3 complex with three mac, respectively, al and ms present. Th III a, m, p series with three mic (a1-3), two mes (a6-7), three mic (m4, m6-6p), three mes $(\mathbf{m 6 e}, \mathbf{m} 7-7 \mathbf{e})$, four mic $(\mathbf{p 1 - 3}, \mathbf{p 6})$ respectively. Ratio Th II: III = 1.04-1.36: $1(\mathrm{n}=5)$, holotype $=$ 1.05: 1 .

Abdomen dorsal chaetotaxy (Fig. 12B-C). Abd I a, m series with one (a5) and six (m2-6e) mic respectively, ms present. Abd II a, m, p series with two mic (a6-7), two mac (m3, m5), three mic (p5-7) respectively, el mic and as present; $\mathbf{a 5}$ and $\mathbf{m} \mathbf{2}$ bothriotricha surrounded by five and four fan-shaped chaetae respectively. Abd III a, m, p series with one mic (a7), three fan-shaped chaetae (a2-3, a6), two mic (m7i-7), three mac (m3, am6, pm6), three mic (p6e, p7i-7), one mac (p6) chaetae respectively; $\mathbf{a 5}, \mathbf{m} \mathbf{2}$ and $\mathbf{m 5}$ bothriotricha with six, two and three fan-shaped chaetae respectively, as sens elongated, ms present. Abd IV A-Fe series with two mic (A1, A6), two mac (A3, A5), one mic (B1), one mes (B6), two mac (B4-5), four mic (C1-4), three mic (T1, T5-6), one mes (T7), five mic (D1-3, De3), one mes (D3p), one mic (E4p2), one mes (E4p), three mac 
(E1-3), one mic (Ee12), two mes (Ee10-11), one mac (Ee9), one mic (F1), two mes (F3, F3p), one mac (F2), one mic (Fe2), three mes (Fe3-5) chaetae, respectively; T2, T4 and E4 bothriotricha surrounded by five and two (T3) fan-shaped chaetae respectively; ps and as present, and at least six supernumerary sens with uncertain homology ' $s$ ' (Fig. 7A); Abd. IV posteriorly with four psp. Abd $\mathrm{V} \mathbf{a}, \mathbf{m}, \mathbf{p}$ series with two mic (a1, a3), one mes (a6), one mac (a5), two mes (m5a, m5e), three mac (m2-3, m5), five mic (p3a-6ae), one mic (p6e) two mes (ap6-pp6), four mac (p1, p3-5) chaetae, respectively; as, acc.p4-5 present. Ratio Abd III: IV=1: 3.70-4.37 ( $\mathrm{n}=5)$, holotype = 1: 4.37 . Legs. Trochanteral organ diamond shape with about 20 spine-like chaetae, plus 2 psp one external and one on distal vertex of Omt (Fig. 13A). Unguis outer side with one paired tooth straight and not developed on proximal third; inner lamella wide with four teeth, basal pair subequal, b.p. not reaching the m.t. apex, m.t. just after the distal half, a.t. present. Unguiculus with lamellae smooth and lanceolate (a.i., a.e., p.i.), except p.e. slightly serrate (Fig. 13B); ratio unguis: unguiculus = 1.56-1.79: $1(\mathrm{n}=5)$, holotype $=1.56: 1$. Tibiotarsal smooth chaetae about $0.9 \times$ smaller than unguiculus; tenent hair capitate and about $0.55 \times$ smaller than unguis outer lamella.

Collophore (Fig. 13C). Anterior side with 12 ciliate, apically acuminate chaetae, five proximal, four subdistal (as mes) and three distal mac; lateral flap with 11 chaetae, five ciliate in the proximal row and six smooth in the distal row.

Furcula. Covered with ciliate chaetae, spine-like chaetae and scales. Manubrial plate with four ciliate chaetae (two inner mac) and three psp (Fig. 13D). Dens posterior face with two or more longitudinal rows of spine-like chaetae about 24 external and 25 internal, external spines larger and thinner than internal ones. Mucro with four teeth, ratio width: length $=0.29$ (holotype).

Etymology. Species named after Dr. Bruno C. Bellini in recognition of his work on Brazilian Collembola.

Remarks. Trogolaphysa bellinii sp. nov. resembles $T$. bessoni, T. epitychia sp. nov., and $T$. mariecurieae sp. nov. by $0+0$ eyes (T. bellinii sp. nov. rarely with $2+2$ eyes), Th II with $3+3$ mac, and Th III without mac, but can be distinguished by presenting Abd IV with 4+4 central mac (A3,

A5, B4-5); T. epitychia sp. nov. with $3+3$ central mac on Abd IV, T. mariecurieae sp. nov. with $2+2$ central mac on Abd IV. 


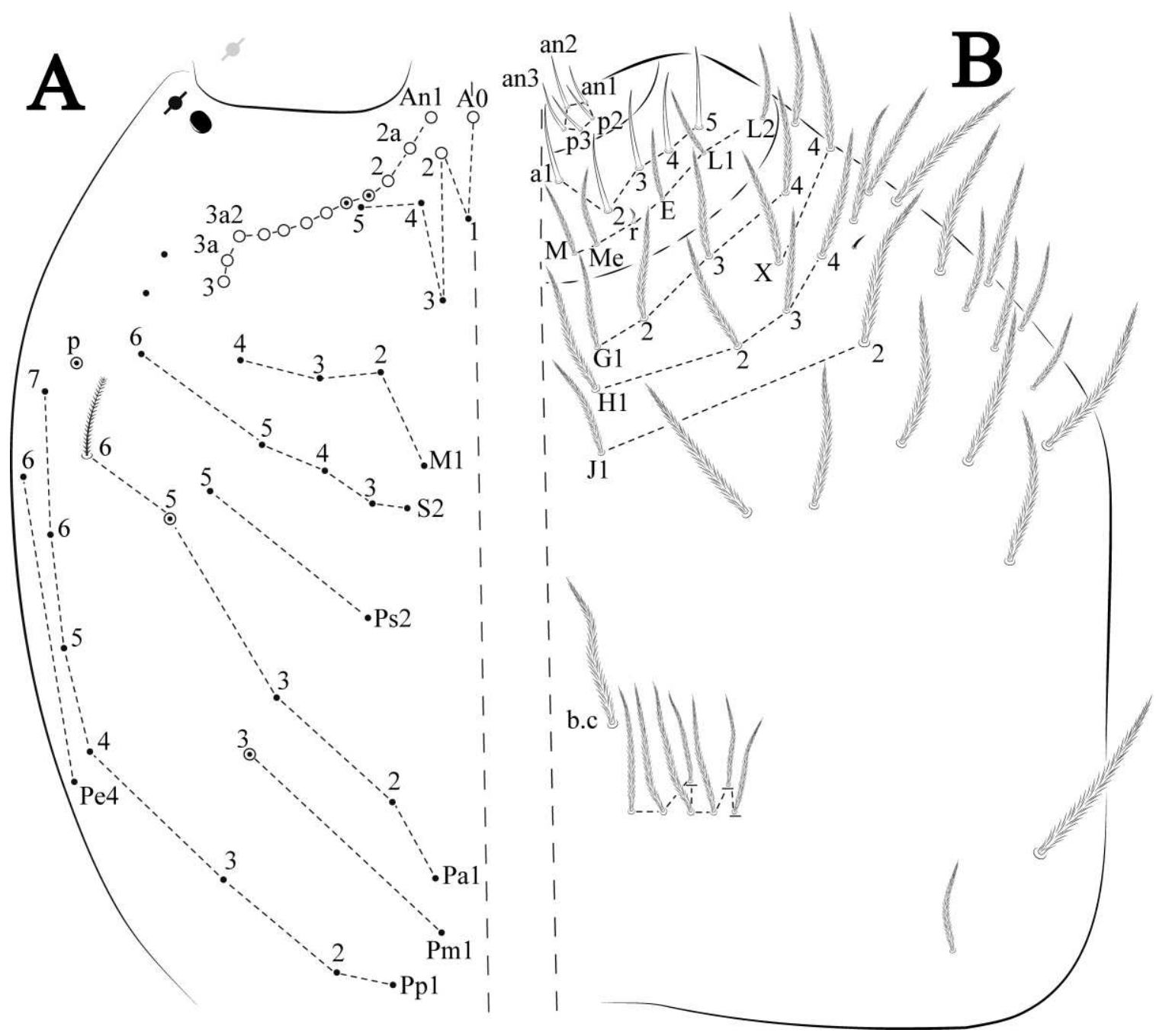

Figure 11. Trogolaphysa bellinii sp. nov.: A) Head dorsal chaetotaxy; B) labial proximal chaetae, basomedial and basolateral labial fields and postlabial chaetotaxy. Black cut circle, pseudopore; Gray cut circle pseudopore at the under surface. 


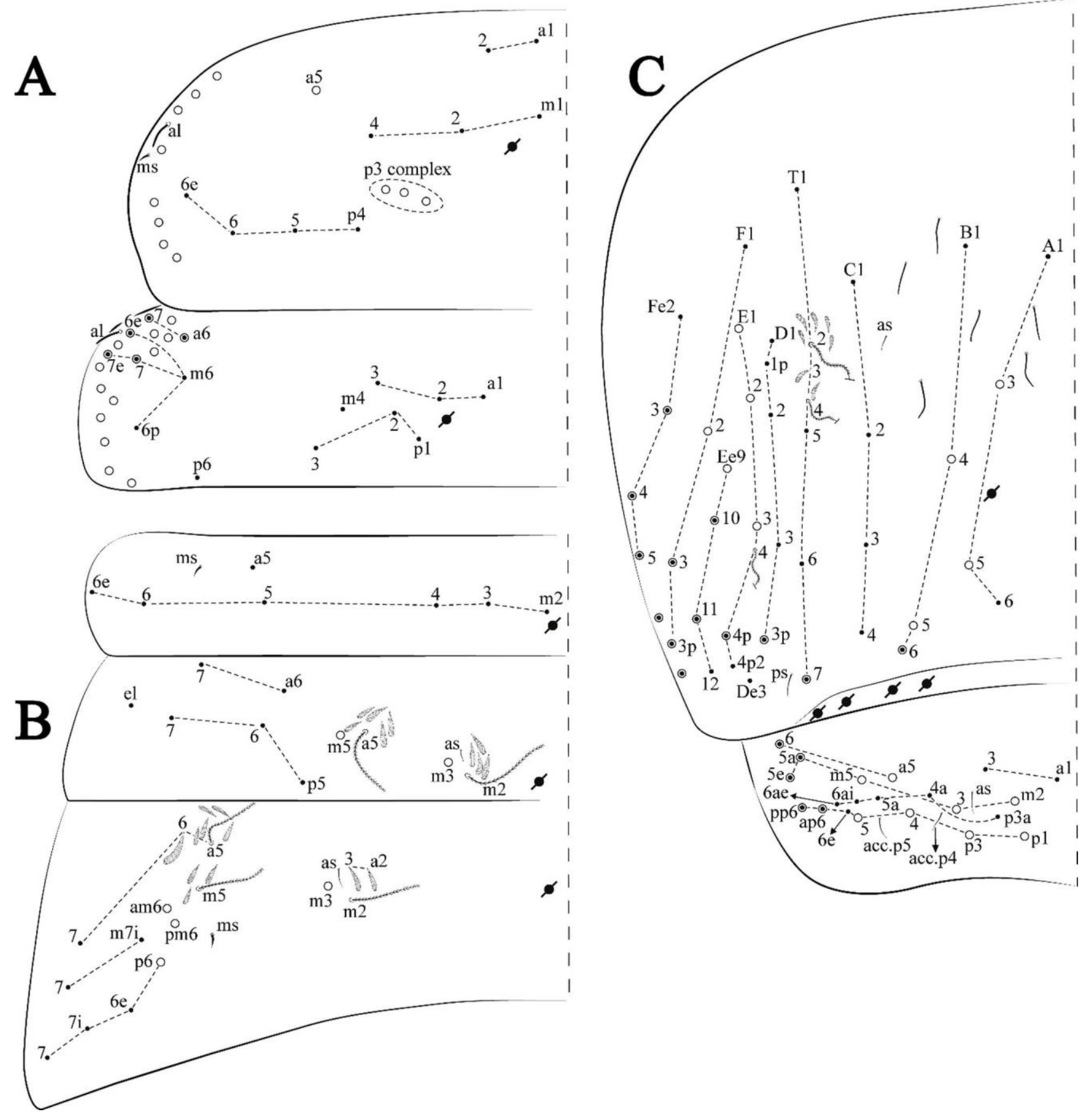

Figure 12. Trogolaphysa bellinii sp. nov.: Dorsal chaetotaxy: A) Th II-III; B) Abd I-III; C) Abd IV-V. 


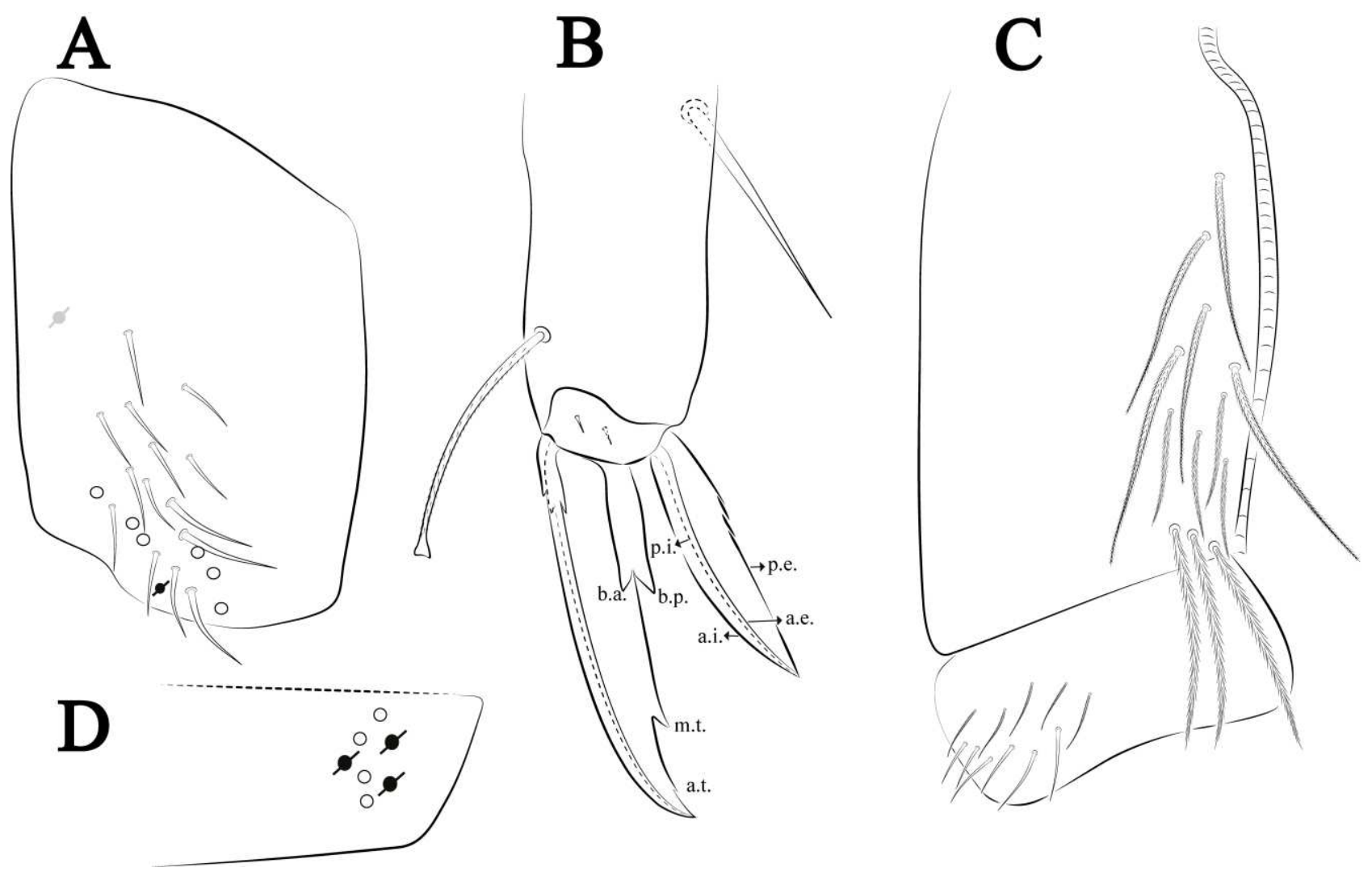

Figure 13. Trogolaphysa bellinii sp. nov.: A) Trochanteral organ; B) Distal tibiotarsus and empodial complex III (anterior view); C) Manubrial plate; D) Antero-lateral view of collophore chaetotaxy. 
Trogolaphysa lacerta sp. nov. Lima, Oliveira \& Zeppelini

Figures 14-16, Tables 1-2

Type material. Holotype male in slide (10311/CRFS-UEPB): Brazil, Minas Gerais State, Conceição do Rio Acima municipality, cave GAND-115, next to "Lapa do Calango", 2004'08.4"S,

4340'09.9"W, 10.ii-20.iii.2014, Carste team coll. Paratypes in slides (10312, 10309/CRFS-UEPB): 2 males, same data as holotype. Paratypes in slides (10313, 10314/CRFS-UEPB donated to MNJR): 2 females, same data as holotype. Additional records see S1.

Description. Total length (head + trunk) of specimens $1.31-2.43 \mathrm{~mm}(\mathrm{n}=5)$, holotype $1.86 \mathrm{~mm}$. Head. Ratio antennae: trunk $=1: 1.33-1.46(\mathrm{n}=2)$, holotype $=1: 1.46$; Ant III shorter than Ant II; Ant segments ratio, I: II, III, IV = 1: 1.78-2.05: 1.5-1.64: 2.64-2.83, holotype =1: 1.80: 1.64: 2.64 . Antennal chaetotaxy (no represented): Ant IV dorsally and ventrally with several short ciliate mic and mac, and finger-shaped sens, dorsally with a longitudinal row with about five rod sens, ventrally with one subapical-organ and several wrinkly sens (Fig. 3A); Ant III dorsally and ventrally with several short ciliate mic and mac, and finger-shaped sens, dorsally without modified sens, ventrally with one apical psp, one apical wrinkly sens on, apical organ with two coffee beanlike sens, and one rod sens (Fig. 3A); Ant II dorsally and ventrally with several short ciliate mic and mac, dorsally with three sub-apical finger-shaped sens, one wrinkly sens and two apical rod sens, ventrally with one apical psp, one longitudinal external row with two subapical wrinkly sens and two medial finger-shaped sens (Fig. 3A); and Ant I dorsally and ventrally with several short ciliate mic and mac, dorsally with three basal spine-like sens, ventrally with four basal spine-like sens, about five smooth mic and several finger-shaped sens (Fig. 3A). Eyes 0+0, rarely 3+3. Head dorsal chaetotaxy (Fig. 14A) with 15 An (An1a-3), six A (A0-5), four M (M1-4), five S (S2-6), two Ps (Ps2, Ps5), four Pa (Pa1-2, Pa4-5), two Pm (Pm1, Pm3), seven Pp (Pp1-7), and two Pe (Pe4, Pe6) chaetae; Pm3, Pa5 and Pp7 as mes, An1a-3a with 11 mac plus four meso, A0 and A2 as mac; interocular $\mathbf{p}$ mes present. Basomedian and basolateral labial fields with $\mathbf{a 1 - 5}$ smooth, M, Me, E and L1-2 ciliate, $\mathbf{r}$ reduced (Fig. 14B). Ventral chaetotaxy with 36-38 ciliate chaetae and 1 reduced lateral spine; postlabial G1-4; X, X4; H1-4; J1-2, chaetae b.c. present and a collar row of three to five mes chaetae distally (Fig. 14B). Prelabral chaetae ciliate. Labral chaetae smooth, no modifications. Labial papilla $\mathbf{E}$ with l.p. finger-shaped and surpassing the base of apical appendage. Labial proximal chaetae smooth (an1-3, p2-3) and subequal in length (Fig. 14B). Maxillary palp with t.a. smooth and $1.28 \times$ larger than t.a.

Thorax dorsal chaetotaxy (Fig. 15A). Th II a, m, p series with two mic (a1-2), one mac (a5), three mic (m1-2, m4) and four mic (p4-6e), p3 complex with six mac, respectively, al and ms present. Th III a, m, p series with three mic (a1-3), two mes (a6-7), three mic (m4, m6-6p), three mes $(\mathbf{m 6 e}, \mathbf{m} 7-7 \mathbf{e})$, four mic $(\mathbf{p 1 - 3}, \mathbf{p 6})$ respectively. Ratio Th II: III = 1.09-1.46: $1(\mathrm{n}=5)$, holotype $=$ 1.09: 1 .

Abdomen dorsal chaetotaxy (Fig. 15B-C). Abd I m series with six (m2-6e) mic respectively, ms present. Abd II a, m, p series with two mic (a6-7), two mac (m3, m5), three mic (p5-7) respectively, el mic and as present; $\mathbf{a 5}$ and $\mathbf{m} \mathbf{2}$ bothriotricha surrounded by four and two fan-shaped chaetae respectively. Abd III a, m, p series with one mic (a7), three fan-shaped chaetae (a2-3, a6), two mic (m7i-7), three mac (m3, am6, pm6), four mic (p6e, p7i-7p), one mac (p6) chaetae respectively; $\mathbf{a 5}, \mathbf{m} \mathbf{2}$ and $\mathbf{m} \mathbf{5}$ bothriotricha with seven, two and four fan-shaped chaetae respectively, as sens elongated, ms present. Abd IV A-Fe series with four mic (A1, A5-6, Ae1), one mac (A3), one mic (B1), one mes (B6), two mac (B4-5), four mic (C1-4), five mic (T1, T3, T5-7), five mic (D1-3, De3), one mes (D3p), one mic (E4p2), one mes (E4p), three mac (E1-3), 
one mic (Ee12), two mes (Ee10-11), one mac (Ee9), one mic (F1), two mes (F3-3p), one mac (F2), one mic (Fe2), three mes (Fe3-5) chaetae, respectively; T2, T4 and E4 bothriotricha surrounded by four and one fan-shaped chaetae respectively; ps and as present, and at least six supernumerary sens with uncertain homology 's'(Fig. 7A); Abd. IV posteriorly with five to six psp. Abd V a, m, p series with two mic (a1, a3), one mes (a6), one mac (a5), two mes (m5a, m5e), three mac (m2-3, m5), five mic (p3a-6ae), one mic (p6e) two mes (ap6-pp6), four mac (p1, p3-5) chaetae, respectively; as, acc.p4-5 present. Ratio Abd III: IV = 1: 3.70-4.37 ( $\mathrm{n}=5)$, holotype $=1$ : 4.37.

Legs. Trochanteral organ diamond shape with about 24 spine-like chaetae, plus 2 psp one external and one on distal vertex of Omt (Fig. 16A). Unguis outer side with one paired tooth straight and not developed on proximal third; inner lamella wide with four teeth, basal pair subequal, b.p. not reaching the m.t. apex, m.t. just after the distal half, a.t. present. Unguiculus with all lamellae smooth and lanceolate (a.i., a.e., p.i., p.e.) (Fig. 16B); ratio unguis: unguiculus $=1: 1.50-1.79(\mathrm{n}=$ $5)$, holotype $=1: 1.75$. Tibiotarsal smooth chaetae about $0.7 \times$ smaller than unguiculus; tenent hair slightly acuminate and about $0.44 \times$ smaller than unguis outer lamella.

Collophore (Fig. 16C). Anterior side with 10 ciliate, apically acuminate chaetae, five proximal (thinner); three subdistal and two distal mac; lateral flap with 11 chaetae, five ciliate in the proximal row and six smooth in the distal row.

Furcula. Covered with ciliate chaetae, spine-like chaetae and scales. Manubrial plate with four ciliate chaetae (two inner mac) and three psp (Fig. 16D). Dens posterior face with two or more longitudinal rows of spine-like chaetae about 50 external and 37 internal, external spines larger and thinner than internal ones. Mucro with four teeth, ratio width: length $=0.31(n=5)$.

Etymology. Lacerta from Latin means lizard, in allusion to the name of the cave where this species was found, Lapa do Calango (cave of the Calango), which is a small lizard common in this region. Remarks. Trogolaphysa lacerta sp. nov. The new species resembles T. caripensis, T. ernesti, T. piracurucaensis, $T$. formosensis and $T$. dandarae sp. nov. by the number of mac in Th II $\mathbf{p} 3$ complex (6+6), but is easily distinguished by the head $\mathbf{m} \mathbf{2}$ and $\mathbf{s 5}$ mic (T. caripensis, T. ernesti, T. formosensis, T. piracurucaensis as mac) and Th III without mac (T. dandarae sp. nov. $3+3$ ). 


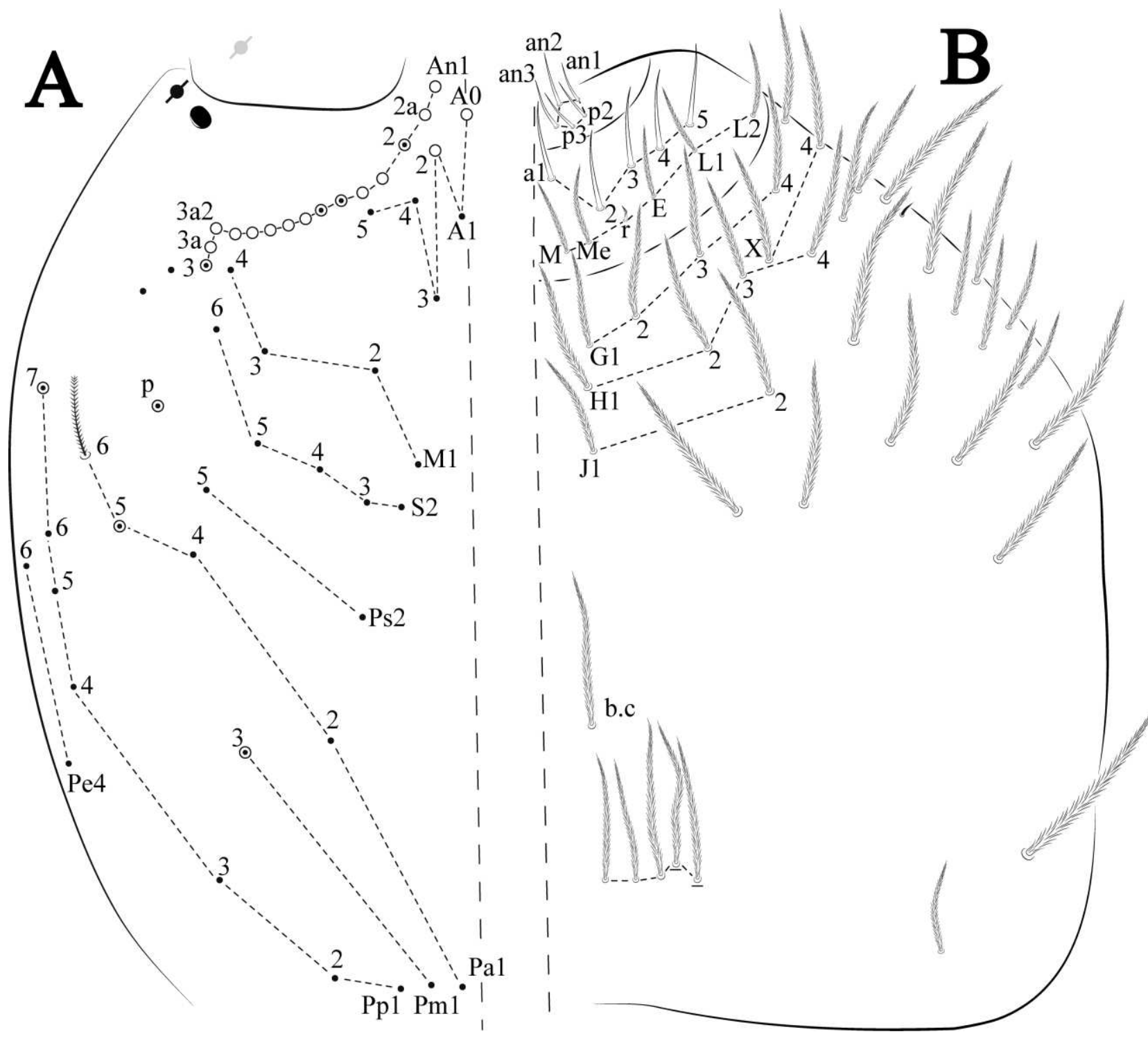

Figure 14. Trogolaphysa lacerta sp. nov.: A) Head dorsal chaetotaxy; B) labial proximal chaetae, basomedial and basolateral labial fields and postlabial chaetotaxy. Black cut circle, pseudopore; Gray cut circle pseudopore at the under surface. 


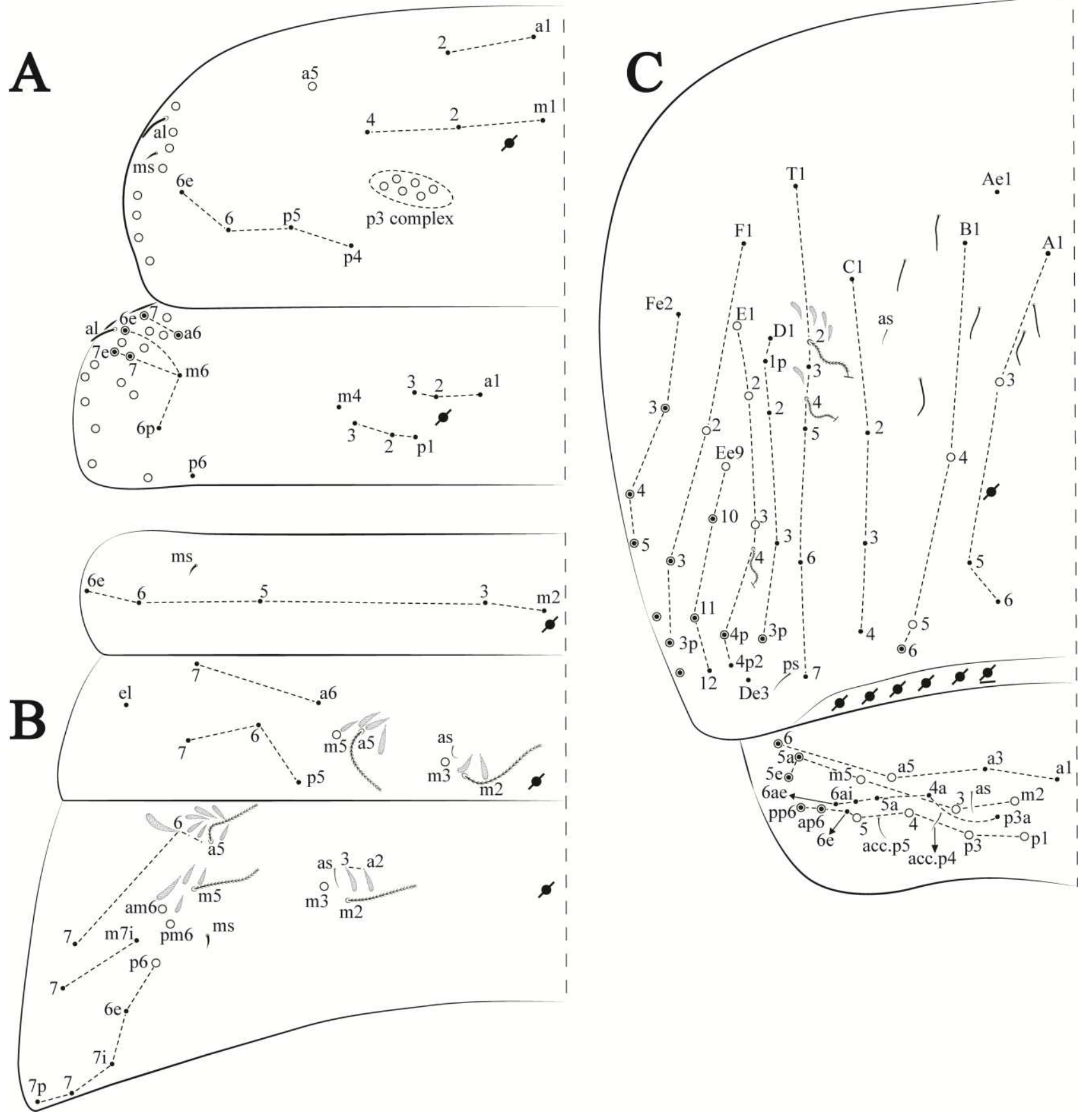

Figure 15. Trogolaphysa lacerta sp. nov.: Dorsal chaetotaxy. A) Th II-III; B) Abd I-III; C) Abd IV-V. 


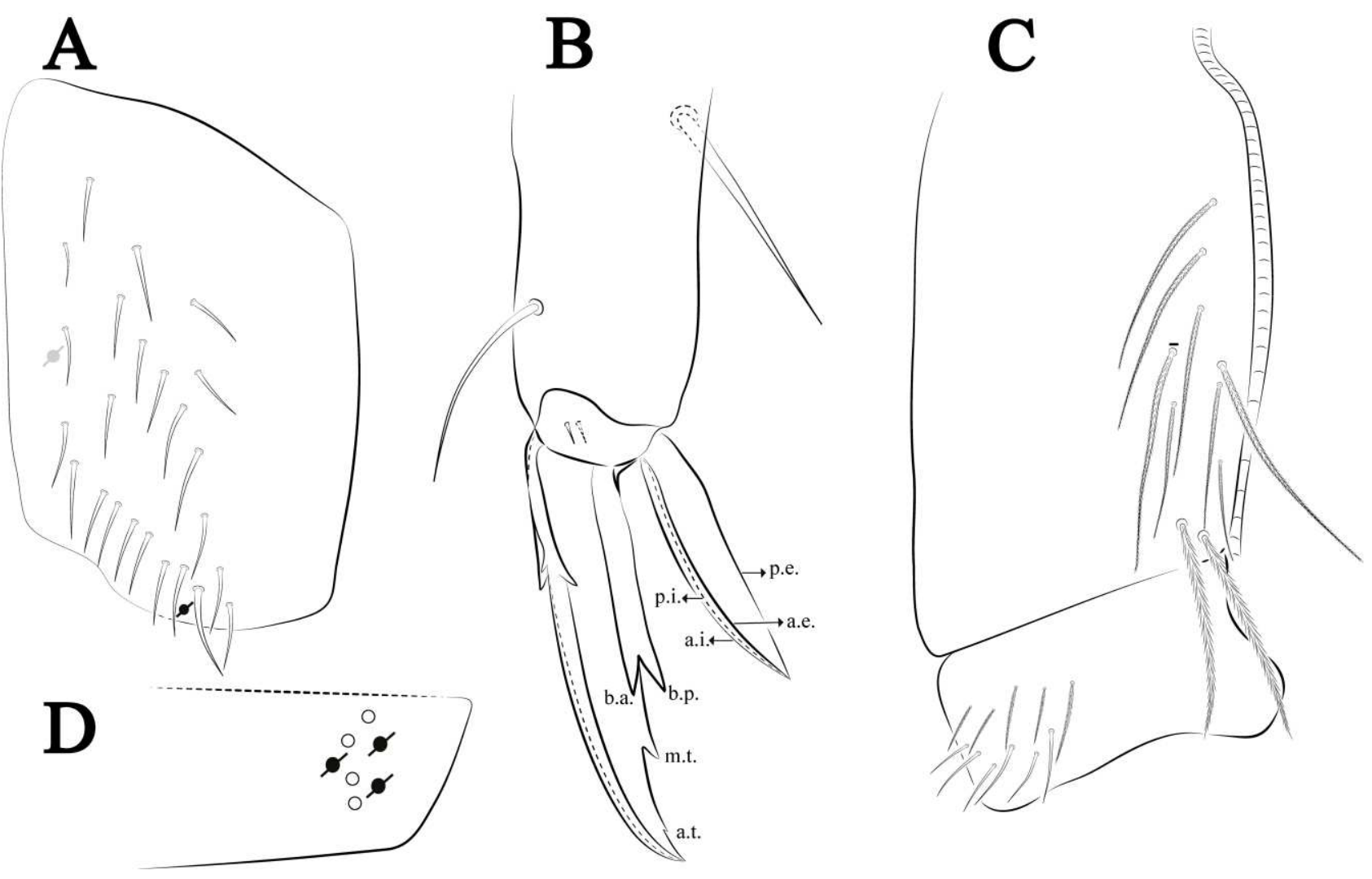

Figure 16. Trogolaphysa lacerta sp. nov.: A) Trochanteral organ; B) Distal tibiotarsus and empodial complex III (anterior view); C) Manubrial plate; D) Antero-lateral view of collophore chaetotaxy. 
Trogolaphysa chapelensis sp. nov. Lima, Oliveira \& Zeppelini

Figures 17-19, Tables 1-2

Type material. Holotype female in slide (4550/CRFS-UEPB): Brazil, Minas Gerais State, Rio Acima municipality, cave Gruta-2d7, next to "Morro do Chapéu" 2007'42.1"S, 4354'26.2"W, 02 10.viii.2011, Andrade et al. coll. Paratypes in slides (4551-4553/CRFS-UEPB): 3 females, Brazil, Minas Gerais State, Rio Acima municipality, cave Gruta-7d7, Qd7, 9d7 respectively, 2007'42.1"S, 4354'26.7"W, 29.iii-01.vi.2011, Andrade et al. coll. Paratype in slide (4603/CRFS-UEPB donated to MNJR): 1 female, Brazil, Minas Gerais State, Rio Acima municipality, cave Gruta Qd7, 2009'46.1"S, 4349'36.2"W, 925 m, 29.iii-01.vi.2011, Andrade et al. Coll. Additional records see S1.

Description. Total length (head + trunk) 1.21-2.22 mm $(\mathrm{n}=5)$, holotype $2.22 \mathrm{~mm}$. Head. Ratio antennae: trunk $=1: 1.31-1.16(\mathrm{n}=3)$, holotype $=1: 1.16$; Ant III shorter than Ant II; Ant segments ratio, I: II, III, IV $=1: 1.66-1.85,1.65-1.78,2.95-3.76$, holotype $=1: 1.66,1.65$, 2.95. Antennal chaetotaxy (no represented): Ant IV dorsally and ventrally with several short ciliate mic and mac, and finger-shaped sens, dorsally with about six rod sens on longitudinal row, ventrally with one subapical-organ and about three subapical wrinkly sens (Fig. 3A); Ant III dorsally and ventrally with several short ciliate mic and mac, and finger-shaped sens, dorsally without modified sens, ventrally with one apical psp, one apical wrinkly sens, apical organ with two coffee bean-like sens, and one rod sens (Fig. 3A); Ant II dorsally and ventrally with several short ciliate mic and mac, dorsally with about three sub-apical finger-shaped sens and about three apical rod sens, ventrally with one apical psp, one longitudinal external row with four wrinkly sens (Fig. 3A); and Ant I dorsally and ventrally with several short ciliate mic and mac, dorsally with three basal spinelike sens, ventrally with four basal spine-like sens, about three smooth mic and several fingershaped sens (Fig. 3A). Eyes 0+0. Head dorsal chaetotaxy (Fig. 17A) with 15 An (An1a-3), six A (A0-5), four M (M1-4), five S (S2-6), two Ps (Ps2, Ps5), four Pa (Pa1-5), two Pm (Pm1, Pm3), seven Pp (Pp1-7), and two Pe (Pe4, Pe6) chaetae; Pm3 and Pa5 as mes, An1a-3a with 13 mac plus two mes, $\mathbf{A 0}$ and $\mathbf{A 2}$ as mac; interocular $\mathbf{p}$ mic present. Basomedian and basolateral labial fields with a1-5 smooth, M, Me, E and L1-2 ciliate, $\mathbf{r}$ reduced (Fig. 17B). Ventral chaetotaxy with 29 ciliate chaetae; postlabial G1-4; X, X4; H1-4; J1-2, chaetae b.c. present and a collar row of six mes chaetae distally (Fig. 17B). Prelabral chaetae ciliate. Labral chaetae smooth, no modifications. Labial papilla $\mathbf{E}$ with l.p. finger-shaped and surpassing the base of apical appendage. Labial proximal chaetae smooth (an1-3, p2-3) and subequal in length (Fig. 17B). Maxillary palp with t.a. smooth and $1.17 \times$ larger than b.c.

Thorax dorsal chaetotaxy (Fig. 18A). Th II a, m, p series with two mic (a1-2), one mac (a5), three mic (m1-2, m4) and four mic (p4-6e), p3 complex with four mac, respectively, al and ms present. Th III a, m, p series with three mic (a1-3), two mes (a6-7), two mic (m4-6p), four mes (m6-6e, $\mathbf{m 7 - 7 e}$ ), four mic (p1-3, p6) respectively. Ratio Th II: III = 1.10-1.31: $1(\mathrm{n}=4)$, holotype = 1.10: 1 . Abdomen dorsal chaetotaxy (Figs. 18B-C). Abd I a, m series with one (a5) and six (m2-6e) mic respectively, ms present. Abd II a, m, p series with two mic (a6-7), two mac (m3, m5), three mic (p5-7) respectively, el mic and as present; $\mathbf{a 5}$ and $\mathbf{m} \mathbf{2}$ bothriotricha surrounded by five and four fan-shaped chaetae respectively. Abd III a, m, p series with one mic (a7), three fan-shaped chaetae (a2-3, a6), two mic (m7i-7), three mac (m3, am6, pm6), three mic (p6e, p7i-7), one mac (p6) chaetae respectively; $\mathbf{a 5}, \mathbf{m} \mathbf{2}$ and $\mathbf{m} \mathbf{5}$ bothriotricha with six, two and three fan-shaped chaetae respectively, as sens elongated, ms present. Abd IV A-Fe series with three mic (A1, A6, Ae1), two mac (A3, A5), one mic (B1), one mes (B6), two mac (B4-5), four mic (C1-4), three mic (T1, T5- 
6), one mes (T7), five mic (D1-3, De3), one mes (D3p), one mic (E4p2), one mes (E4p), three mac (E1-3), one mic (Ee12), two mes (Ee10-11), one mac (Ee9), one mic (F1), two mes (F3-3p), one mac (F2), one mic (Fe2), three mes (Fe3-5) chaetae, respectively; T2, T4 and E4 bothriotricha surrounded by four and two (T3) fan-shaped chaetae respectively; ps and as present, and at least six supernumerary sens with uncertain homology ' $s$ ' (Fig. 7A); Abd. IV posteriorly with nine psp. Abd $\mathrm{V} \mathbf{a}, \mathbf{m}, \mathbf{p}$ series with two mic (a1, a3), one mes (a6), one mac (a5), two mes (m5a, m5e), three mac (m2-3, m5), five mic (p3a-6ae), one mic (p6e) two mes (ap6-pp6), four mac (p1, p3-5) chaetae, respectively; as, acc.p4-5 present. Ratio Abd III: IV=1: 3.46-5.80 ( $\mathrm{n}=5)$, holotype = 1: 5.80 .

Legs. Trochanteral organ diamond shape with about 23 spine-like chaetae, plus 2 psp one external and one on distal vertex of Omt (Fig. 19A). Unguis outer side with one paired tooth straight and not developed on proximal third; inner lamella wide with four teeth, basal pair subequal, b.p. not reaching the m.t. apex, m.t. just after the distal half, a.t. present. Unguiculus with lamellae smooth and lanceolate (a.i., a.e., p.i.), except p.e. slightly serrate (Fig. 18B); ratio unguis: unguiculus $=1$ : $1.63-1.84(\mathrm{n}=5)$, holotype $=1: 1.79$. Tibiotarsal smooth chaetae about $0.8 \times$ smaller than unguiculus; tenent hair capitate and about $0.52 \times$ smaller unguis outer lamella. Collophore (Fig. 19C). Anterior side with 13 ciliate, apically acuminate chaetae, seven proximal (thinner); four subdistal and two distal mac; lateral flap with 11 chaetae, five ciliate in the proximal row and six smooth in the distal row.

Furcula. Covered with ciliate chaetae, spine-like chaetae and scales. Manubrial plate with four ciliate chaetae (two inner mac) and three psp (Fig. 19D). Dens posterior face with two or more longitudinal rows of spine-like chaetae about 70 external and 30 internal, external spines larger and thinner than internal ones. Mucro with four teeth, ratio width: length $=0.33(n=5)$. Etymology. Species named after Type locality Morro do Chapeu.

Remarks. Trogolaphysa chapelensis sp. nov. resembles $T$. jacobyi, $T$. caripensis, $T$. bessoni, and $T$. belizeana by te absence of eyes ( $0+0$ eyes) but is easily distinguished by presenting $4+4$ mac in Th II p3 complex (2-3+2-3 T. jacobyi; 6+6 T. caripensis; 2+2 T. bessoni; 2-4+2-4 T. belizeana), and 9+9 psp posterior Abd IV (4+4 T. belizeana). 


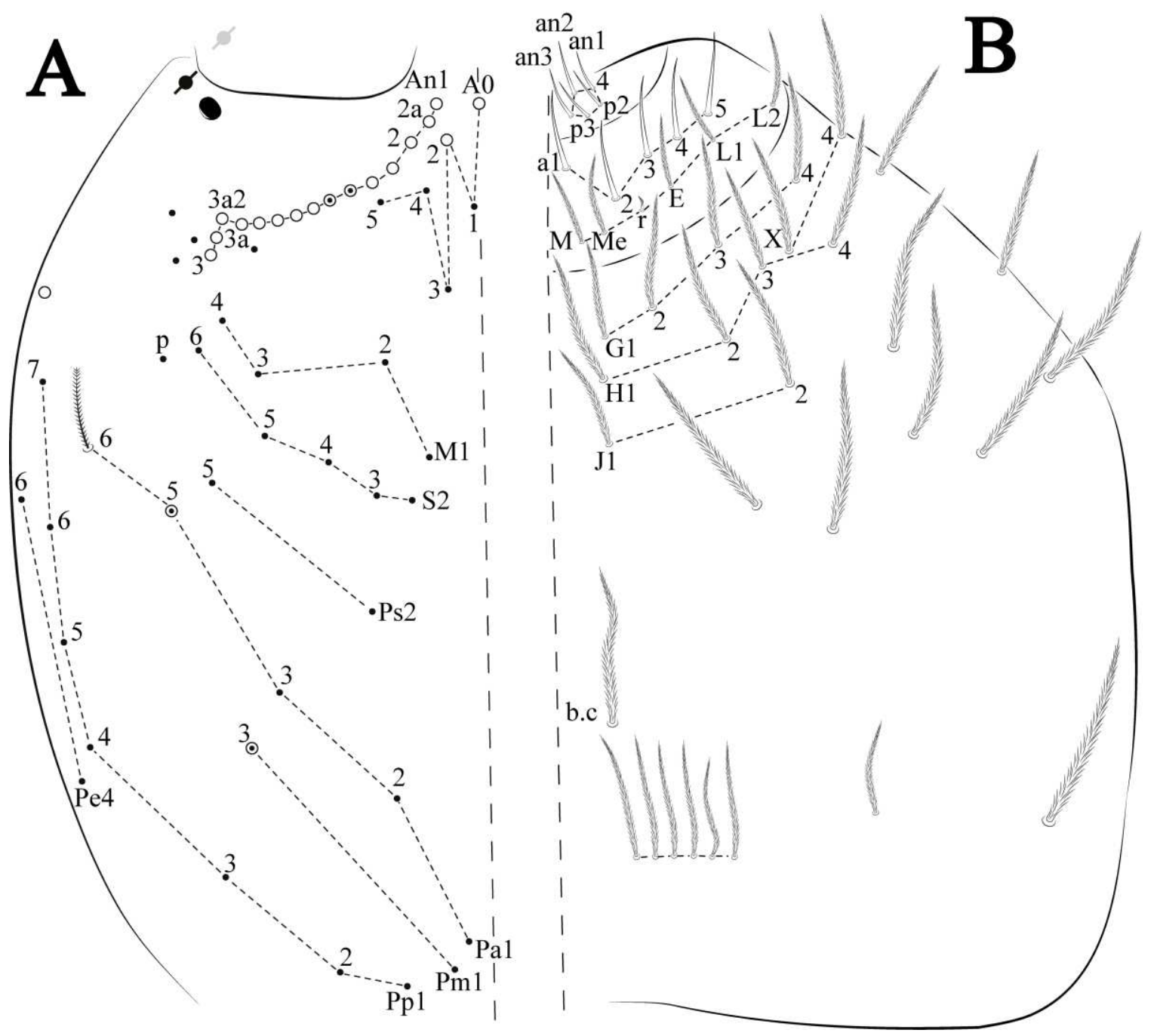

Figure 17. Trogolaphysa chapelensis sp. nov.: A) Head dorsal chaetotaxy; B) labial proximal chaetae, basomedial and basolateral labial fields and postlabial chaetotaxy. Black cut circle, pseudopore; Gray cut circle pseudopore at the under surface. 


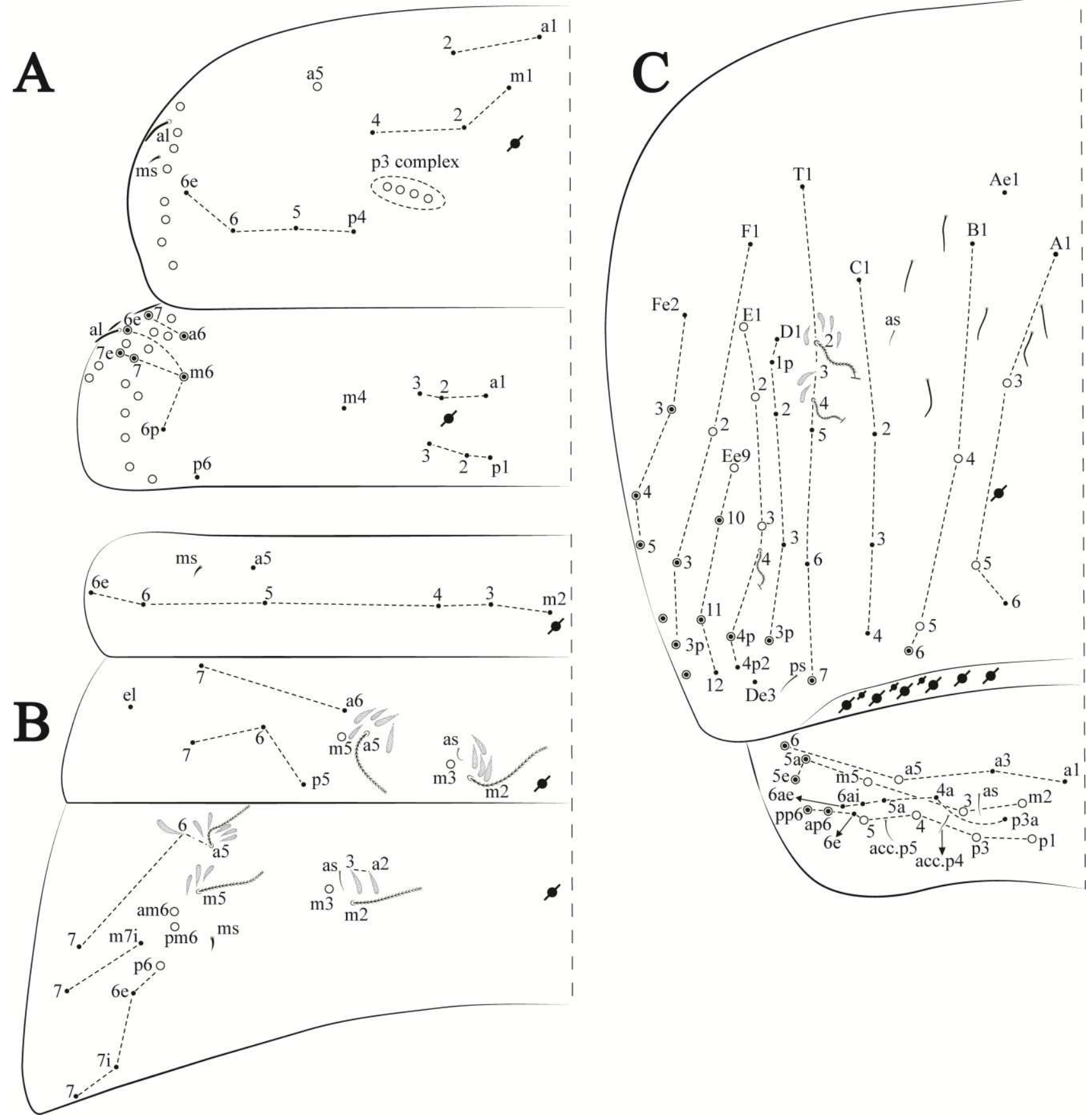

Figure 18. Trogolaphysa chapelensis sp. nov.: Dorsal chaetotaxy. A) Th II-III; B) Abd I-III; C) Abd IV-V. 

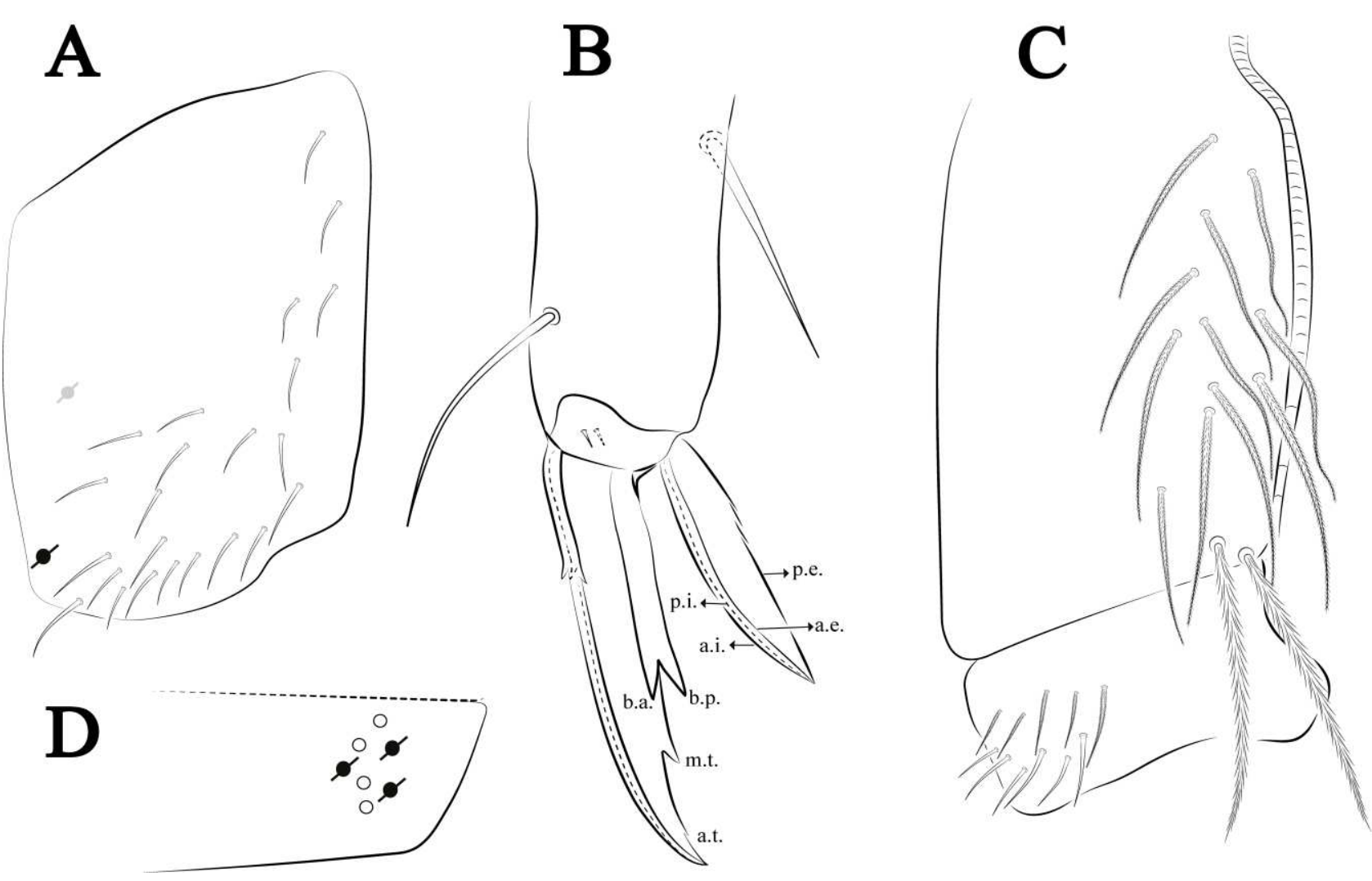

Figure 19. Trogolaphysa chapelensis sp. nov.: A) Trochanteral organ; B) Distal tibiotarsus and empodial complex III (anterior view); C) Manubrial plate; D) Antero-lateral view of collophore chaetotaxy. 
Trogolaphysa crystallensis sp. nov. Oliveira, Lima \& Zeppelini

Figures 20-22, Tables 1-2

Type material. Holotype female in slide (16252/CRFS-UEPB): Brazil, Minas Gerais State, Mariana municipality, cave LOC-0090, next to “Cachoeira Crystal”, 20²0'20.8”'S, 4323'44.3”W, 1114.xi.2019, Carste team coll. Paratype in slide (16251/CRFS-UEPB): female, same data as holotype. Paratype in slide (16254/CRFS-UEPB donated to MNJR): female, same data as holotype. Additional records see S1.

Description. Total length (head + trunk) of specimens 1.40-1.68 mm $(\mathrm{n}=3)$, holotype $1.68 \mathrm{~mm}$. Head. Ratio antennae: trunk $=1: 1.24-2.30(\mathrm{n}=2)$, holotype $=1: 1.24$; Ant III shorter than Ant II length; Ant segments ratio as I: II, III, IV = 1: 1.72-1.78, 1.58-1.64, 3.11-3.14, holotype = 1: 1.78, $1.64,3.14$. Antennal chaetotaxy (no represented): Ant IV dorsally and ventrally with several short ciliate mic and mac, and finger-shaped sens, dorsally with about three rod sens on longitudinal row, ventrally with one subapical-organ and several wrinkly sens (Fig. 3A); Ant III dorsally and ventrally with several short ciliate mic and mac, and finger-shaped sens, dorsally without modified sens, ventrally with one apical psp, about three wrinkly sens on external longitudinal row, apical organ with two rod sens, and one finger-shaped sens (Fig. 3A); Ant II dorsally and ventrally with several short ciliate mic and mac, dorsally with three sub-apical finger-shaped sens and one wrinkly sens, ventrally with one apical psp (Fig. 3A); and Ant I dorsally and ventrally with several short ciliate mic and mac, dorsally with three basal spine-like sens, ventrally with four basal spine-like sens, about three smooth mic and several finger-shaped sens (Fig. 3A). Eyes 0+0. Head dorsal chaetotaxy (Fig. 20A) with 12-13 An (An1a-3), six A (A0-5), four M (M1-4), five S (S2-6), two Ps (Ps2, Ps5), four Pa (Pa1-5), two Pm (Pm1, Pm3), seven Pp (Pp1-7), and two Pe (Pe4, Pe6) chaetae; Pa5, Pm3 and Pp7 as mes, An1a-3a, A0 and A2 as mac; interocular p mes present. Basomedian and basolateral labial fields with a1 $-\mathbf{5}$ smooth, $\mathbf{M}, \mathbf{M e}, \mathbf{E}$ and $\mathbf{L 1}-\mathbf{2}$ ciliate, $\mathbf{r}$ reduced (Fig. 20B). Ventral chaetotaxy with 33-35 ciliate chaetae and one reduced lateral spine; postlabial G1-4; X, X4; H1-4; J1-2, chaetae b.c. present and a collar row of four to six mes chaetae distally (Fig. 20B). Prelabral chaetae ciliate. Labral chaetae smooth, no modifications. Labial papilla $\mathbf{E}$ with l.p. finger-shaped and surpassing the base of apical appendage. Labial proximal chaetae smooth (an1-3, p2-3) and subequal in length (Fig. 20B). Maxillary palp with t.a. smooth and $1.43 \times$ larger than b.c.

Thorax dorsal chaetotaxy (Fig. 21A). Th II a, m, p series with two mic (a1-2), one mac (a5), three mic (m1-2, m4) and four mic (p4-6e), p3 complex with five mac, respectively, al and ms present. Th III a, m, p series with two mic (a1-2), two mes (a6-7), theree mic (m4, m6-6p), three mes $(\mathbf{m 6 e}, \mathbf{m} 7-7 \mathbf{e})$, four mic $(\mathbf{p 1}-\mathbf{3}, \mathbf{p 6})$ respectively. Ratio Th II: III = 1.05-1.27: $1(\mathrm{n}=3)$, holotype $=$ 1.05: 1 .

Abdomen dorsal chaetotaxy (Figs. 21B-C). Abd I a, m series with one (a5) and six (m2-6e) mic respectively, ms present. Abd II a, m, p series with two mic (a6-7), two mac (m3, m5), three mic (p5-7) respectively, el mic and as present; $\mathbf{a 5}$ and $\mathbf{m} \mathbf{2}$ bothriotricha surrounded by four and two fanshaped chaetae respectively. Abd III a, m, p series with one mic (a7), three fan-shaped chaetae (a23, a6), two mic (m7i-7), three mac (m3, am6, pm6), three mic (p6e, p7i-7), one mac (p6) chaetae respectively, $\mathbf{a 5}, \mathbf{m} \mathbf{2}$ and $\mathbf{m 5}$ bothriotricha with six, two and three fan-shaped chaetae respectively, as sens elongated, ms present. Abd IV A-Fe series with three mic (A1, A6, Ae1), two mac (A3, A5), one mic (B1), one mes (B6), two mac (B4-5), four mic (C1-4), three mic (T1, T5-7), five mic (D1-1p, D3-3p, De3), one mes (D2), two mes (E4p-4p2), three mac (E1-3), four mes (Ee9-12), one mic (F1), three mes (F2-3p), one mic (Fe2), three mes (Fe3-5) chaetae, respectively; T2, T4 
and $\mathbf{E 4}$ bothriotricha surrounded by three and two (T3) fan-shaped chaetae respectively; ps and as present, and at least 14 supernumerary sens with uncertain homology ' $s$ ' (Fig. 7A); Abd. IV posteriorly with three psp. Abd V a, m, p series with two mic (a1, a3), one mes (a6), one mac (a5), two mes (m5a, m5e), three mac (m2-3, m5), five mic (p3a-P6ae), three mes (p6e-pp6), four mac (p1, p3-5) chaetae, respectively; as and acc.p4-5 present. Ratio Abd III: IV = 1: 4.06-4.51 (n=3), holotype $=1: 4.51$.

Legs. Trochanteral organ diamond shape with about 18 spine-like chaetae, plus 2 psp one external and one on distal vertex of Omt (Fig. 22A). Unguis outer side with one paired tooth straight and not developed on proximal third; inner lamella wide with three teeth, basal pair subequal, b.p. little larger, but not reaching the m.t. apex, m.t. just after the distal half, a.t. absent. Unguiculus with all lamellae smooth and lanceolate (a.i., a.e., p.i., p.e.) (Fig. 22B); ratio unguis: unguiculus $=1.48-$ 1.79: $1(\mathrm{n}=3)$, holotype $=1.48: 1$. Tibiotarsal smooth chaetae about $0.8 \times$ smaller than unguiculus; tenent hair acuminate and about $0.5 \times$ smaller than unguis outer lamella.

Collophore (Fig. 22C). Anterior side with 10 ciliate, apically acuminate chaetae, six proximal, two subdistal (as mes) and two distal mac; lateral flap with 11 chaetae, five ciliate in the proximal row and six smooth in the distal row.

Furcula. Covered with ciliate chaetae, spine-like chaetae and scales. Manubrial plate with four ciliate chaetae (two inner mac) and three psp (Fig. 22D). Dens posterior face with two or more longitudinal rows of spine-like chaetae about 60 external and 28 internal, external spines larger and thinner than internal ones. Mucro with four teeth, ratio width: length $=0.31$ (holotype). Etymology. Species named after Type locality Cachoeira Crystal (Portuguese for Crystal falls). Remarks. Trogolaphysa crystallensis sp. nov. resembles T. barroca $\mathbf{s p . ~ n o v . , ~ T . ~ g i s b e r t a e ~ s p . ~ n o v . , ~}$ T. sotoadamesi sp. nov., T. triocelata and T. zampauloi sp. nov. by the absence of eyes ( $0+0$ eyes) (T. triocelata 3+3 and T. zampauloi sp. nov. eventually 4+4), Th II with 5+5 mac, and Th III without mac. Can be distinguished from $T$. barroca sp. nov., $T$. gisbertae sp. nov., and $T$. sotoadamesi sp. nov. by the Abd IV with 4+4 central mac (A3, A5, B4-5); T. barroca sp. nov., $T$. gisbertae sp. nov., and T. triocelata, with $3+3$ and T. sotoadamesi sp. nov. $2+2$ central mac on Abd IV. Finally, the new species differentiates from $T$. zampauloi $\mathbf{s p . ~ n o v . ~ b y ~ u n p a i r e d ~ l a m e l l a ~ o f ~ u n g u i s ~}$ with one tooth, Omt with about 18 spine-like chaetae, dens external row with about 58 spines on $T$. crystallensis sp. nov. and unpaired lamella of unguis with 2 teeth, Omt with about 26 spine-like chaetae, dens external row with about 30 spines on $T$. zampauloi sp. nov. 


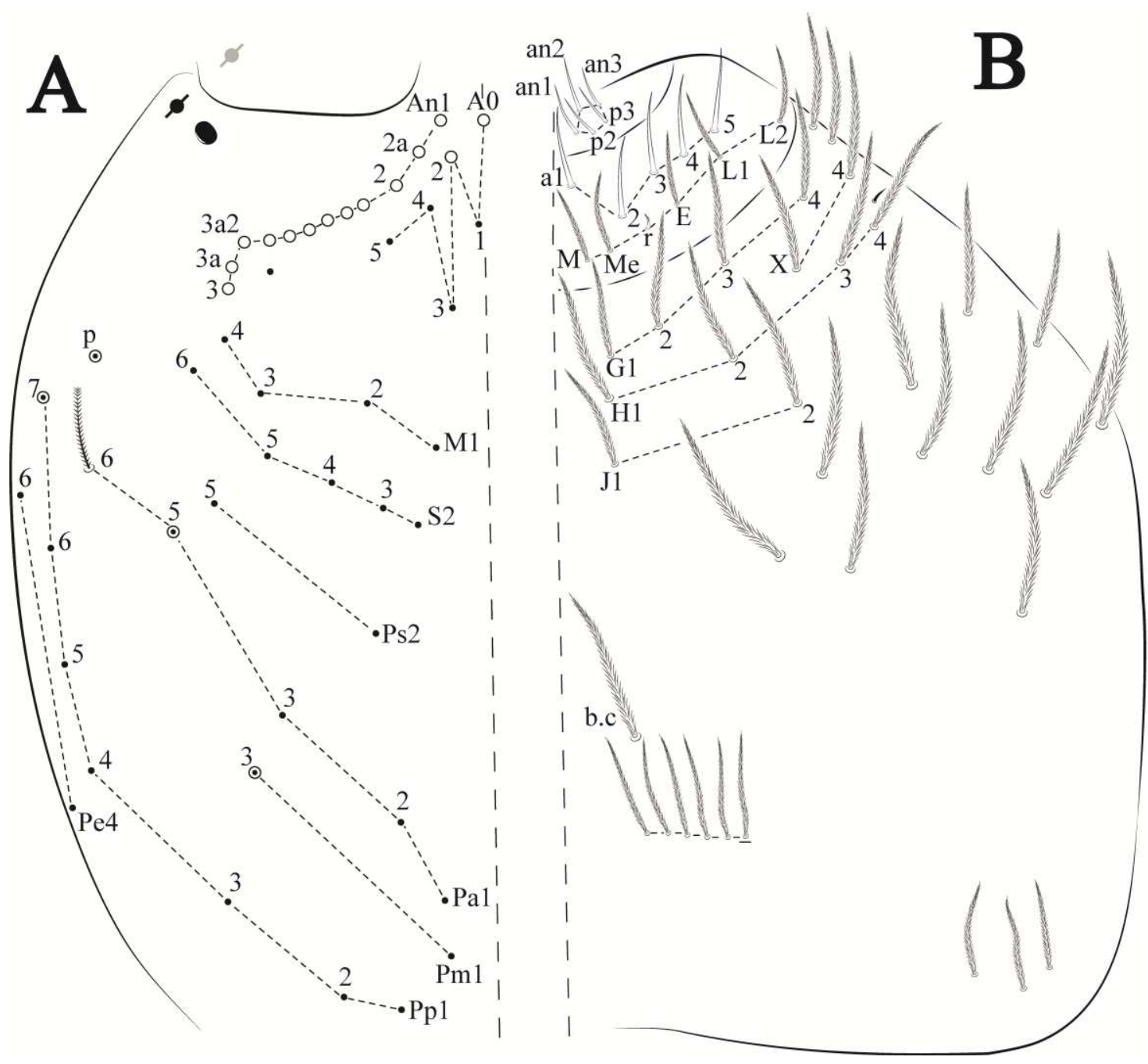

Figure 20. Trogolaphysa crystallensis sp. nov.: A) Head dorsal chaetotaxy; B) labial proximal chaetae, basomedial and basolateral labial fields and postlabial chaetotaxy. Black cut circle, pseudopore; Gray cut circle pseudopore at the under surface. 


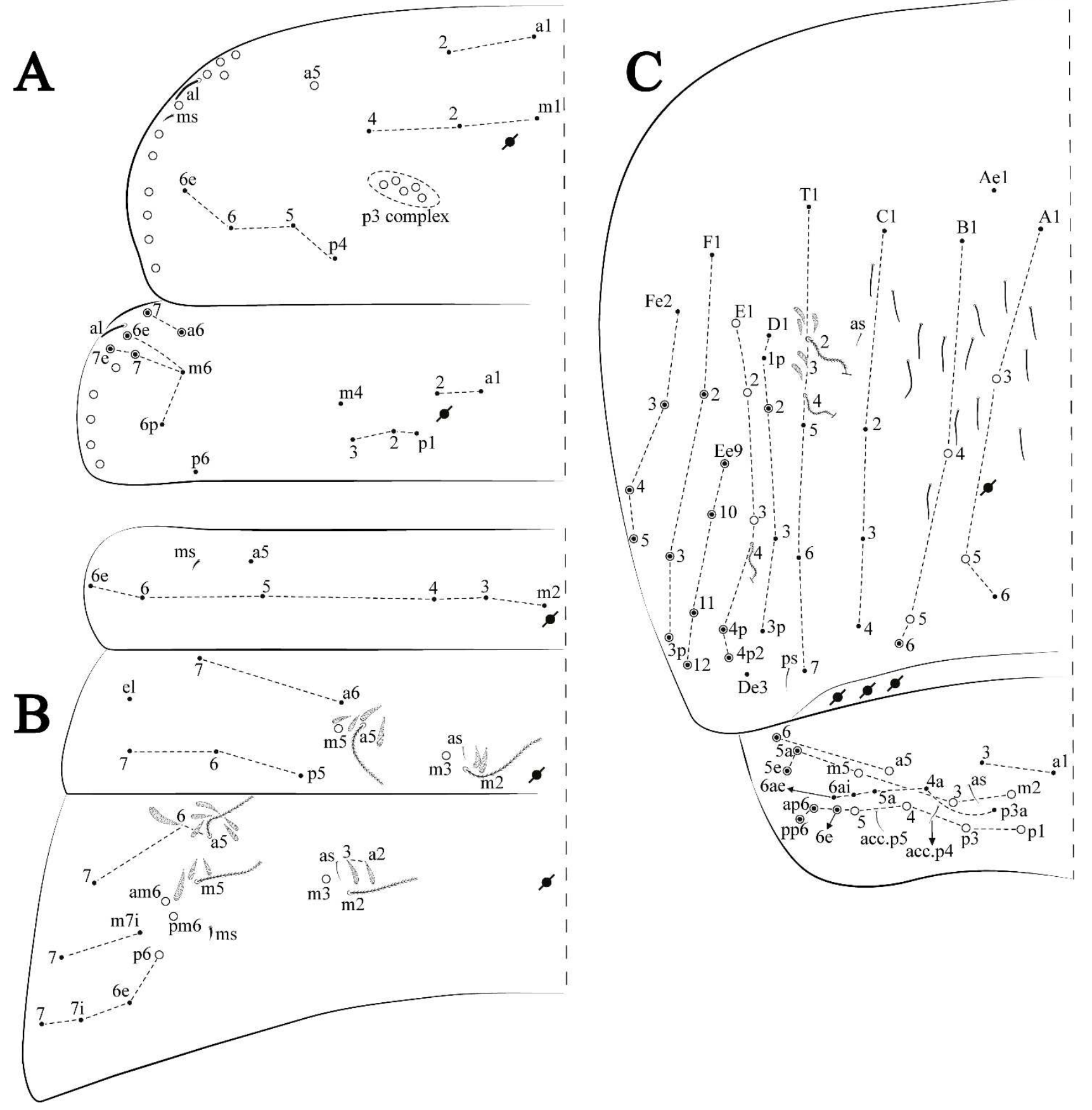

Figure 21. Trogolaphysa crystallensis sp. nov.: Dorsal chaetotaxy. A) Th II-III; B) Abd I-III; C) Abd IV-V. 

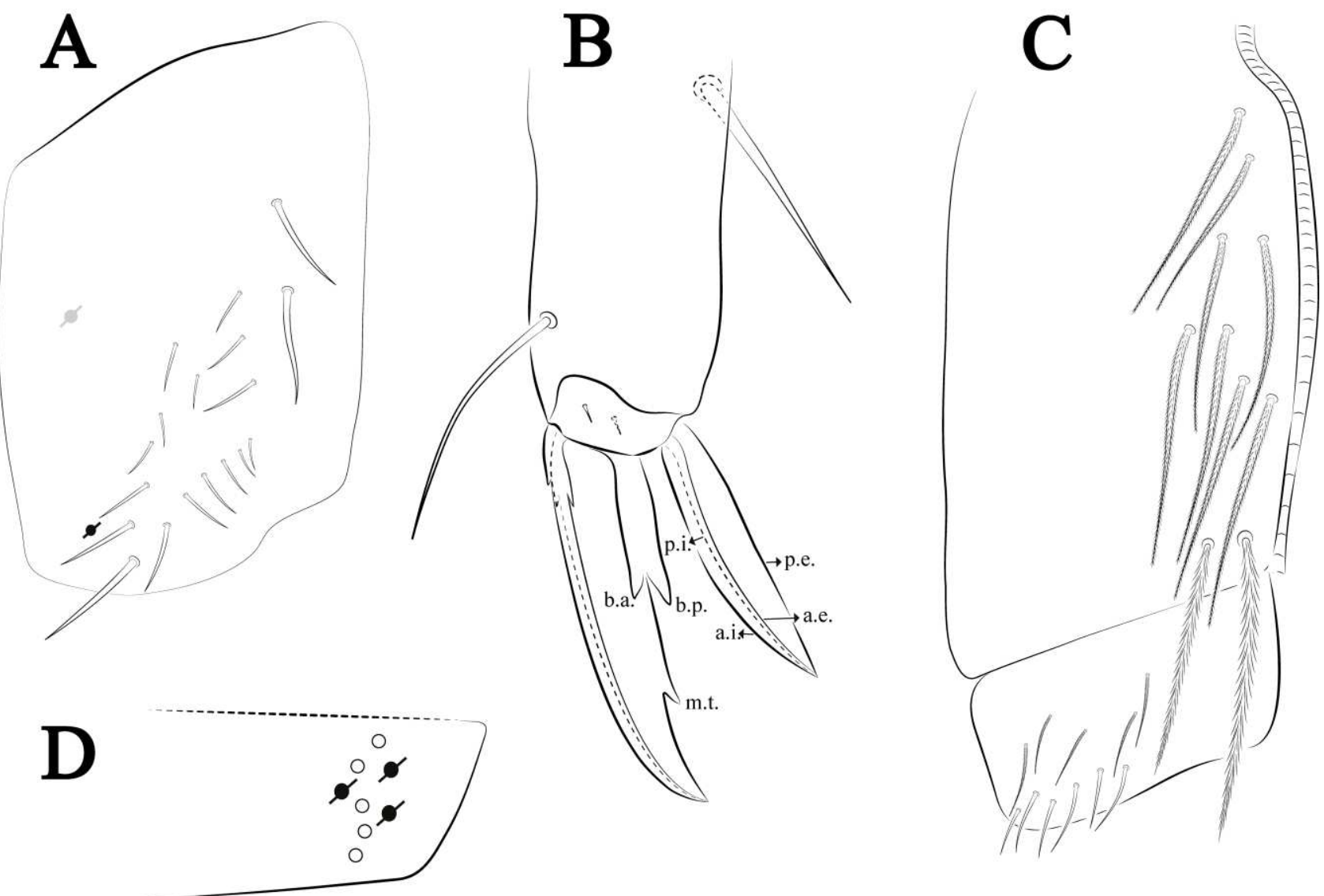

Figure 22. Trogolaphysa crystallensis sp. nov.: A) Trochanteral organ; B) Distal tibiotarsus and empodial complex III (anterior view); C) Manubrial plate; D) Antero-lateral view of collophore chaetotaxy. 
Trogolaphysa sotoadamesi sp. nov. Ferreira, Oliveira \& Zeppelini

Figures 23-25, Tables 1-2

Type material. Holotype male in slide (13162/CRFS-UEPB): Brazil, Minas Gerais State, Mariana municipality, cave ALEA 0003, next to “Mina de Alegria”, 2009'6.81'S, 4329'13.6”'W, 07.ii.2018, Bioespeloeo team coll. Paratypes in slides (13146, 13153/CRFS-UEPB): 2 females, same data as holotype, except 12.vi.2017. Paratype in slide (13173, 13186/CRFS-UEPB donated to MNJR): 2 females, same data as holotype, except 09.vi.2017. Additional records see S1.

Description. Total length (head + trunk) of specimens1.50-1.81 mm $(\mathrm{n}=5)$, holotype $1.50 \mathrm{~mm}$. Head. Ratio antennae: trunk = 1: 1.26-1.45 $(\mathrm{n}=3)$, holotype = 1: 1.38; Ant III shorter than Ant II; Ant segments ratio, I: II, III, IV = 1: 1.78-2.76, 1.52-2.22, 2.61-3.90, holotype = 1: 2.04, 1.68, 3.16. Antennal chaetotaxy (no represented): Ant IV dorsally and ventrally with several short ciliate mic and mac, and finger-shaped sens, dorsally with a longitudinal row with about three rod sens, ventrally with one subapical-organ and with several wrinkly sens (Fig. 3A); Ant III dorsally and ventrally with several short ciliate mic and mac, and finger-shaped sens, dorsally without modified sens, ventrally with one apical psp, several wrinkly sens, apical organ with two coffee bean-like sens, one rod sens and one finger-shaped sens (Fig. 3A); Ant II dorsally and ventrally with several short ciliate mic and mac, dorsally with two sub-apical rod sens and two finger-shaped sens, ventrally with one apical psp and several finger-shaped sens (Fig. 3A); and Ant I dorsally and ventrally with several short ciliate mic and mac, dorsally with three basal spine-like sens, ventrally with about seven basal spine-like sens, about three smooth mic and several finger-shaped sens (Fig. 3A). Eyes 0+0. Head dorsal chaetotaxy (Fig. 23A) with 16 An (An1a-3), six A (A0-5), four M (M1-4), five S (S2-6), two Ps (Ps2, Ps5), four Pa (Pa1-5), two Pm (Pm1, Pm3), seven Pp (Pp17), and two Pe (Pe4, Pe6) chaetae; Pm3 as mes (rarely mic), Pa5 as mes, An1a-3a, A0 and A2 as mac; interocular $\mathbf{p}$ mes present. Basomedian and basolateral labial fields with $\mathbf{a 1}-\mathbf{5}$ smooth, $\mathbf{M}, \mathbf{M e}$, E and L1-2 ciliate, $\mathbf{r}$ reduced (Fig. 23B). Ventral chaetotaxy with 37 ciliate chaetae and 1 reduced lateral spine; postlabial G1-4; X, X4; H1-4; J1-2, chaetae b.c. present and a collar row of six mes chaetae distally (Fig. 23B). Prelabral chaetae ciliate. Labral chaetae smooth, no modifications. Labial papilla $\mathbf{E}$ with l.p. finger-shaped and surpassing the base of apical appendage. Labial proximal chaetae smooth (an1-3, p2-p3) and subequal in length (Fig. 23B). Maxillary palp with t.a. smooth and $1.28 \times$ larger than b.c.

Thorax dorsal chaetotaxy (Fig. 24A). Th II a, m, p series with two mic (a1-2), one mac (a5), three mic (m1-2, m4) and four mic (p4-6e), p3 complex with five mac, respectively, al and ms present. Th III a, m, p series with three mic (a1-3, a6), one mes (a7), four mic (m4, m6-7, m6p), two mes $(\mathbf{m 6 e}, \mathbf{m} 7 \mathbf{e})$, four mic $(\mathbf{p 1}-\mathbf{3}, \mathbf{p 6})$ respectively. Ratio Th II: III $=1.17-1.52: 1(\mathrm{n}=5)$, holotype $=$ 1.03: 1 .

Abdomen dorsal chaetotaxy (Figs. 24B-C). Abd I a, m series with one (a5) and six (m2-6e) mic respectively, ms present. Abd II a, m, p series with two mic (a6-7), two mac (m3, m5), three mic (p5-7) respectively, el mic and as present; $\mathbf{a 5}$ and $\mathbf{m} \mathbf{2}$ bothriotricha surrounded by five and three fan-shaped chaetae respectively. Abd III a, m, p series with one mic (a7), three fan-shaped chaetae (a2-3, a6), two mic (m7i-7), three mac (m3, am6, pm6), three mic (p6e, p7i-7), one mac (p6) chaetae respectively; $\mathbf{a 5}, \mathbf{m} \mathbf{2}$ and $\mathbf{m} \mathbf{5}$ bothriotricha with five, two and three fan-shaped chaetae respectively, as sens elongated, ms present. Abd IV A-Fe series with five mic (A1, A3, A5-6, Ae1), one mic (B1), one mes (B6), two mac (B4-5), four mic (C1-4), two mic (T1, T6), two mes (T5, T7), three mic (D1-2), two mes (D3p, De3), two mes (E4p-p2), three mac (E1-3), one mic (Ee12), three mes (Ee9-11), one mic (F1), two mes (F3-3p), one mac (F2), one mic (Fe2), three mes (Fe3- 
5) chaetae, respectively; T2, T4 and E4 bothriotricha surrounded by four and three (T3) fan-shaped chaetae respectively; $\mathbf{p s}$ and as present, and at least five supernumerary sens with uncertain homology 's' (Fig. 7A); Abd. IV posteriorly with four psp. Abd V a, m, p series with two mic (a1, a3), one mes (a6), one mac (a5), two mes (m5a, m5e), three mac (m2-3, m5), five mic (p3ap6ae), one mic (P6e) two mes (ap6-pp6), four mac (p1, p3-5) chaetae, respectively; as, acc.p4-5 present. Ratio Abd III: IV=1: 5.03-4.42 ( $\mathrm{n}=5)$, holotype = 1: 4.42 .

Legs. Trochanteral organ triangular shape with about 19-21 spine-like chaetae, plus 2 psp one external and one on distal vertex of Omt (Fig. 25A). Unguis outer side with one paired tooth straight and not developed on proximal third; inner lamella wide with two teeth, basal pair unequal, b.p. larger than b.a.; m.t. and a.t. absent. Unguiculus with all lamellae smooth and lanceolate (a.i., a.e., p.i., p.e.) (Fig. 25B); ratio unguis: unguiculus $=1: 1.46-1.91(\mathrm{n}=5)$, holotype $=1: 1.91$. Tibiotarsal smooth chaetae about $0.8 \times$ smaller unguiculus; tenent hair acuminate and about $0.4 \times$ smaller than unguis outer lamella.

Collophore (Fig. 25C). Anterior side with seven ciliate, apically acuminate chaetae, three proximal, two subdistal and two distal mac; lateral flap with nine chaetae, four ciliate in the proximal row and five smooth in the distal row.

Furcula. Covered with ciliate chaetae, spine-like chaetae and scales. Manubrial plate with five ciliate chaetae (two inner mac) and three psp (Fig. 25D). Dens posterior face with two or more longitudinal rows of spine-like chaetae about 35 external and 26 internal, external spines larger and thinner than internal ones. Mucro with four teeth, ratio width: length $=0.39(n=5)$.

Etymology. Species named after Dr. Felipe N. Soto-Adames for his contribution on Collembola taxonomy and systematics.

Remarks. Trogolaphysa sotoadamesi sp. nov. resembles T. barroca $\mathbf{s p . ~ n o v . , ~ T . ~ c r y s t a l l e n s i s ~} \mathbf{s p .}$ nov., T. gisbertae sp. nov., T. zampauloi by $0+0$ eyes ( . zampauloi sp. nov. rarely with $4+4$ eyes), Th II $\mathbf{p} 3$ complex with five mac, Th III without mac, manubrial plate with five ciliate chaetae and mucro with four teeth. The new species $T$. sotoadamesi sp. nov. with $2+2$ central mac on Abd IV differentiates from T. barroca sp. nov., T. gisbertae sp. nov. with 3+3, and T. crystallensis sp. nov., T. zampauloi $\mathbf{s p . ~ n o v . ~ w i t h ~} 4+4$ central mac. 


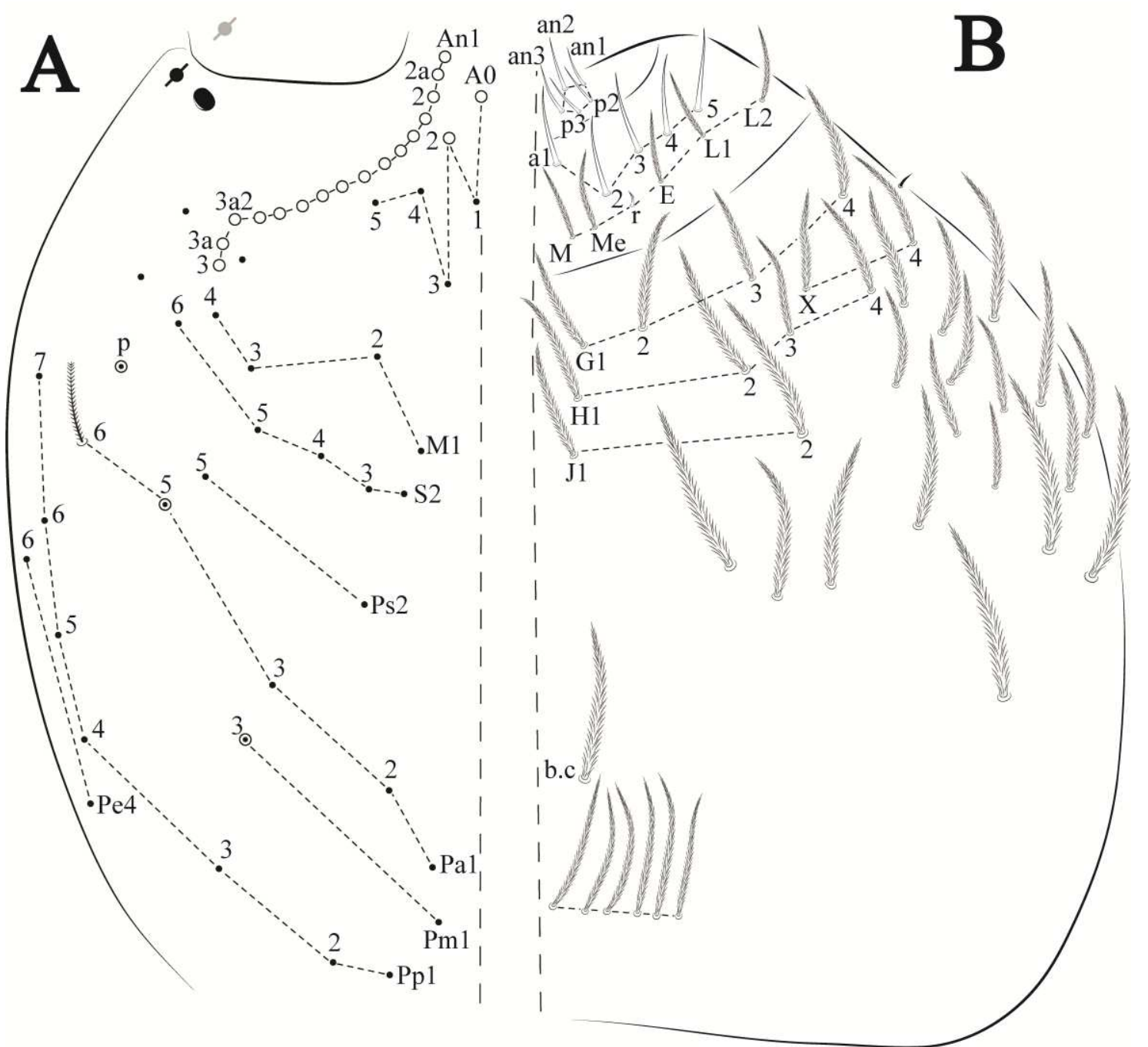

Figure 23. Trogolaphysa sotoadamesi sp. nov.: A) Head dorsal chaetotaxy; B) labial proximal chaetae, basomedial and basolateral labial fields and postlabial chaetotaxy. Black cut circle, pseudopore; Gray cut circle pseudopore at the under surface. 


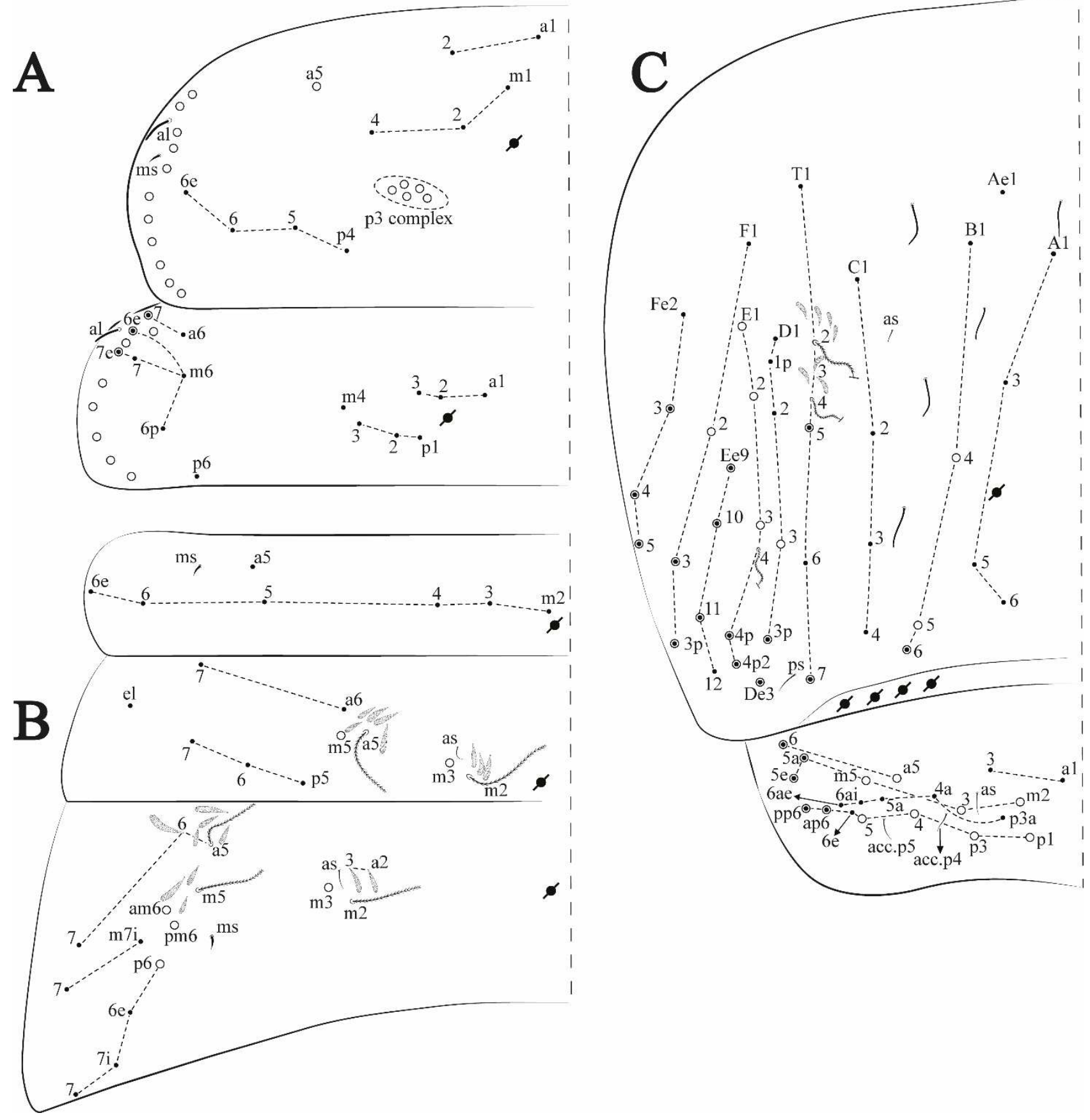

Figure 24. Trogolaphysa sotoadamesi sp. nov.: Dorsal chaetotaxy. A) Th II-III; B) Abd I-III; C) Abd IV-V. 

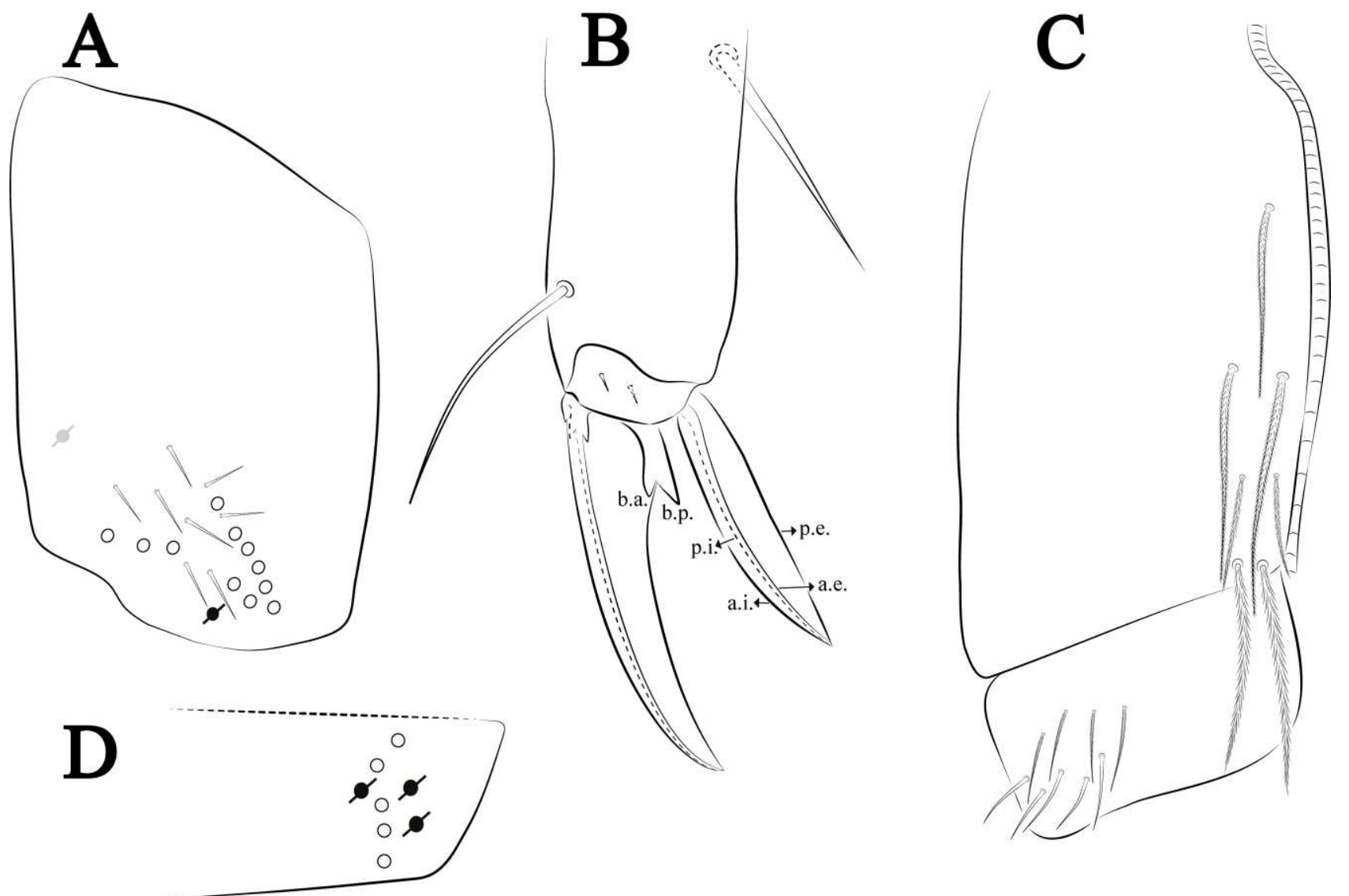

Figure 25. Trogolaphysa sotoadamesi sp. nov.: A) Trochanteral organ; B) Distal tibiotarsus and empodial complex III (anterior view); C) Manubrial plate; D) Antero-lateral view of collophore chaetotaxy. 


\section{Trogolaphysa mariecurieae sp. nov. Ferreira, Oliveira \& Zeppelini}

Figures 26-28, Tables 1-2

Type material. Holotype female in slide (9109/CRFS-UEPB): Brazil, Minas Gerais State, Conceição do Mato Dentro municipality, MSS 10/11, next to "Pico do Soldado" 1900'23.86"S, 4323'41.266”'W, 11-10.ix.2015, Carste team coll. Paratypes in slides (5888, 5857/CRFS-UEPB): 2 females, same data as holotype, except,19-23.v.2014, Soares et al. coll.

Paratype in slide (9222, 10760/CRFS-UEPB donated to MNJR): 2 females, same data as holotype, except 1900'18.72”S, 43²3'30.031'W, 14.x.2015 and 18-20.iv.2016. Additional records see S1.

Description. Total length (head + trunk) of specimens 1.07-1.49 mm ( $\mathrm{n}=5)$, holotype $1.49 \mathrm{~mm}$. Head. Ratio antennae: trunk $=1: 1.69-1.91(\mathrm{n}=2)$, holotype $=1: 1.69$; Ant III shorter than Ant II length; Ant segments ratio, I: II, III, IV = 1: 2.00-2.75, 1.69-2.55, 4.02-5.29, holotype = 1: 2.75, 1.69, 4.02. Antennal chaetotaxy (no represented): Ant IV dorsally and ventrally with several short less ciliate mic and mac, and finger-shaped sens, dorsally with one longitudinal row with about four rod sens, ventrally with one subapical-organ and several wrinkly sens (Fig. 3A); Ant III dorsally and ventrally with several short less ciliate mic and mac, and finger-shaped sens, dorsally without modified sens, ventrally with one apical psp, apical organ with two rod sens (Fig. 3A); Ant II dorsally and ventrally with several short less ciliate mic and mac, dorsally with five apical rod sens, ventrally with one apical psp, about five wrinkly sens on longitudinal external row (Fig. 3A); and Ant I dorsally and ventrally with several short less ciliate mic and mac, dorsally with three basal spine-like sens, ventrally with seven basal spine-like sens, about five smooth mic, and several finger-shaped sens (Fig. 3A). Eyes 0+0. Head dorsal chaetotaxy (Fig. 26A) with 12 An (An1a-3), six A (A0-5), four M (M1-4), five S (S2-6), two Ps (Ps2, Ps5), four Pa (Pa1-3, Pa5), two Pm (Pm1, Pm3), seven Pp (Pp1-7), and two Pe (Pe4, Pe6) chaetae; Pm3 and Pa5 as mes, An1a-3a, $\mathbf{A 0}$ and $\mathbf{A 2}$ as mac; interocular $\mathbf{p}$ mic present. Basomedian and basolateral labial fields with $\mathbf{a 1 - 5}$ smooth, M, Me, E and L1-2 ciliate, $\mathbf{r}$ reduced (Fig. 26B). Ventral chaetotaxy with 34 ciliate chaetae and 1 reduced lateral spine; postlabial G1-4; X, X4; H1-4; J1-2, chaetae b.c. present and a collar row of six mes chaetae distally (Fig. 26B). Prelabral chaetae ciliate. Labral chaetae smooth, no modifications. Labial papilla $\mathbf{E}$ with l.p. finger-shaped and surpassing the base of apical appendage. Labial proximal chaetae smooth (an1-3, p2-3) and subequal in length (Fig. 26B). Maxillary palp with t.a. smooth and $1.13 \times$ larger than b.c.

Thorax dorsal chaetotaxy (Fig. 27A). Th II a, m, p series with two mic (a1-2), one mac (a5), three mic (m1-2, m4) and four mic (p4-6e), p3 complex with three mac, respectively, al and ms present. Th III a, m, p series with three mic (a1-3), two mes (a6-7), three mic (m4-m6p), three mes (m6e, m7-7e), four mic (p1-3, p6) respectively. Ratio Th II: III = 0.85-1.02: $1(\mathrm{n}=4)$, holotype = 0.89: 1 . Abdomen dorsal chaetotaxy (Figs. 27B-C). Abd I a, m series with one (a5) and six (m2-6e) mic respectively, ms present. Abd II a, m, p series with two mic (a6-7), two mac (m3, m5), three mic (p5-7) respectively, el mic and as present; $\mathbf{a 5}$ and $\mathbf{m} \mathbf{2}$ bothriotricha surrounded by four and two fanshaped chaetae respectively. Abd III a, m, p series with one mic (a7), three fan-shaped chaetae (a23, a6), two mic (m7i-7), three mac (m3, am6, pm6), two mic (p6e, p7i), one mac (p6) chaetae respectively; $\mathbf{a 5}, \mathbf{m} \mathbf{2}$ and $\mathbf{m 5}$ bothriotricha with five, two and two fan-shaped chaetae respectively, as sens elongated, ms present. Abd IV A-Fe series with three mic (A1, A6, Ae1), one mac (A4), two mic (B1-2), one mes (B6), one mac (B5), four mic (C1-4), three mic (T1, T5, T7), five mic (D1-3, De3), one mes (D3p), one mic (E4p2), one mes (E4p), three mac (E1-3), one mic (Ee12), two mes (Ee10-11), one mac (Ee9), one mic (F1), three mes (F2-3p), one mic (Fe2), three mes 
(Fe3-5) chaetae, respectively; T2, T4 and E4 bothriotricha surrounded by four and three (T3) fanshaped chaetae respectively; ps and as present, and at least five supernumerary sens with uncertain homology ' $s$ ' (Fig. 7A); Abd. IV posteriorly with four psp. Abd V a, m, p series with two mic (a1, a3), one mes (a6), one mac (a5), five mac (m2-3, m5-5e), five mic (p3a-p6ae), two mes (ap6pp6), four mac (p1, p3-5) chaetae, respectively; as, acc.p4-5 present. Ratio Abd III: IV =1: 4.27$5.91(\mathrm{n}=5)$, holotype $=1: 5.02$.

Legs. Trochanteral organ diamond shape with about 15 spine-like chaetae, plus 2-3 psp one external, one on distal vertex and another (present or absent) on top of posterior spines row of Omt (Fig. 28A). Unguis outer side with one paired tooth straight and not developed on proximal third; inner lamella wide with two teeth, basal pair subequal, b.p. larger than b.a., inner lamella with unpaired small m.t. between b.a. and b.p. and a.t. absent. Unguiculus with all lamellae smooth and truncate (a.i., a.e., p.i., p.e.) (Fig. 28B); ratio unguis: unguiculus $=1.50-1.95: 1(\mathrm{n}=5)$, holotype $=$ 1.95: 1 . Tibiotarsal smooth chaetae about $0.9 \times$ smaller than unguiculus; tenent hair slightly capitate and about $0.6 \times$ smaller than unguis outer lamella.

Collophore (Fig. 28C). Anterior side with eight ciliate, apically acuminate chaetae, six proximal and two distal mac; lateral flap with 13 chaetae, five ciliate in the proximal row and eight smooth in the distal row.

Furcula. Covered with ciliate chaetae, spine-like chaetae and scales. Manubrial plate with four ciliate chaetae (two inner mac) and three psp (Fig. 28D). Dens posterior face with two or more longitudinal rows of spine-like chaetae about 40 external and 22 internal, external spines larger and thinner than internal ones. Mucro with four teeth, ratio width: length $=0.23$ (holotype).

Etymology. Species named after Dr. Marie Skłodowska-Curie for her enormous contribution to science.

Remarks. Trogolaphysa mariecurieae sp. nov. resembles $T$. bellinii sp. nov. $T$. jacobyia and $T$. epitychia sp. nov. by the absence of eyes (T. bellinii sp. nov. rarely with $2+2$ eyes), Th II p3 complex with three mac and with one unpaired tooth on inner lamella of unguis. The new species $T$. mariecurieae sp. nov. (Abd IV with 2+2 mac) differs from $T$. jacobyia, T. epitychia sp. nov. both with Abd IV 3+3, and $T$. bellinii sp. nov. with 4+4 central mac. T. mariecurieae sp. nov. and $T$. bellinii sp. nov. with capitate tenent hair, in contrast with $T$. jacobyia and T. epitychia sp. nov. with acuminated tenant hair. 


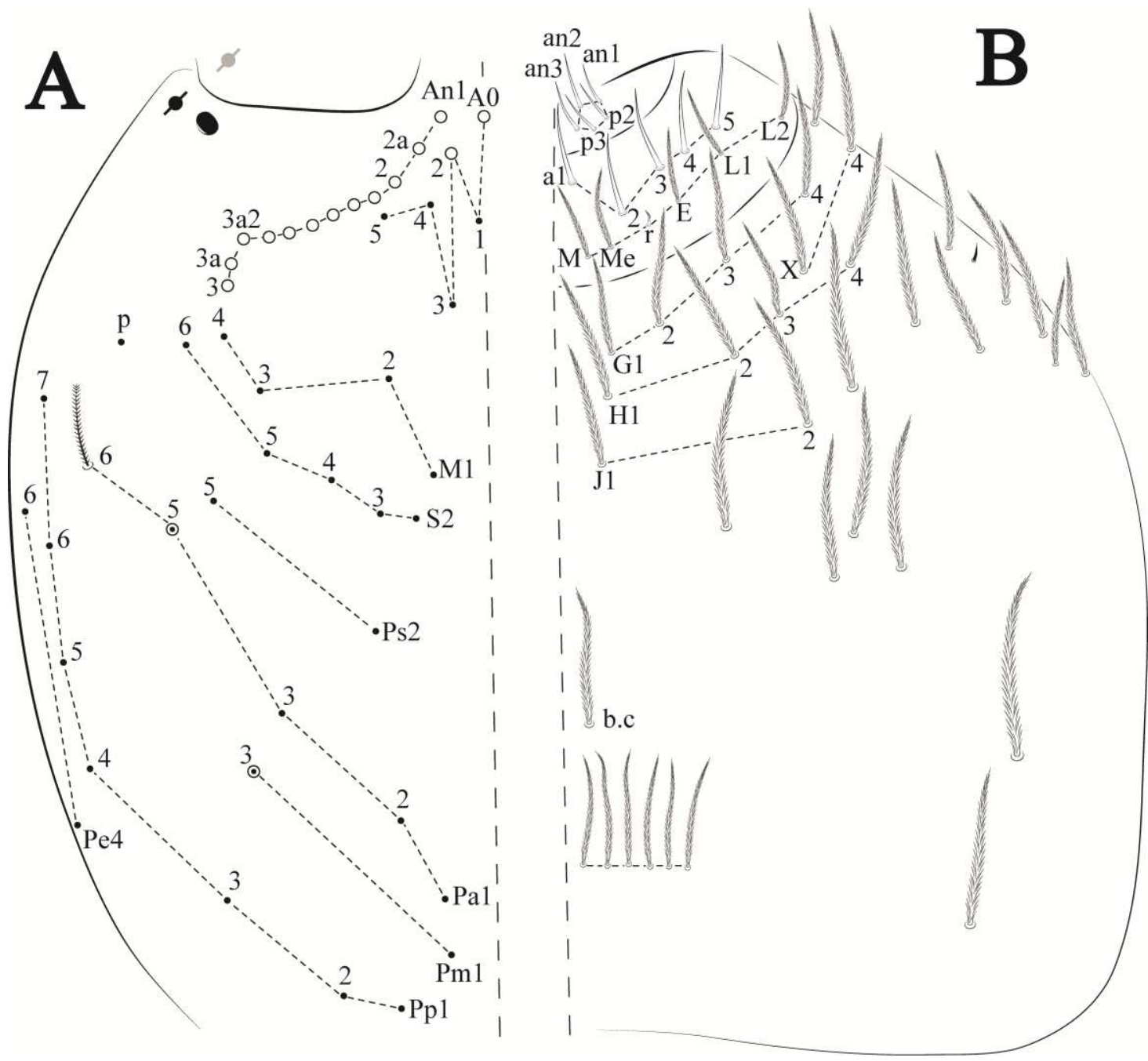

Figure 26. Trogolaphysa mariecurieae sp. nov.: A) Head dorsal chaetotaxy; B) labial proximal chaetae, basomedial and basolateral labial fields and postlabial chaetotaxy. Black cut circle, pseudopore; Gray cut circle pseudopore at the under surface. 


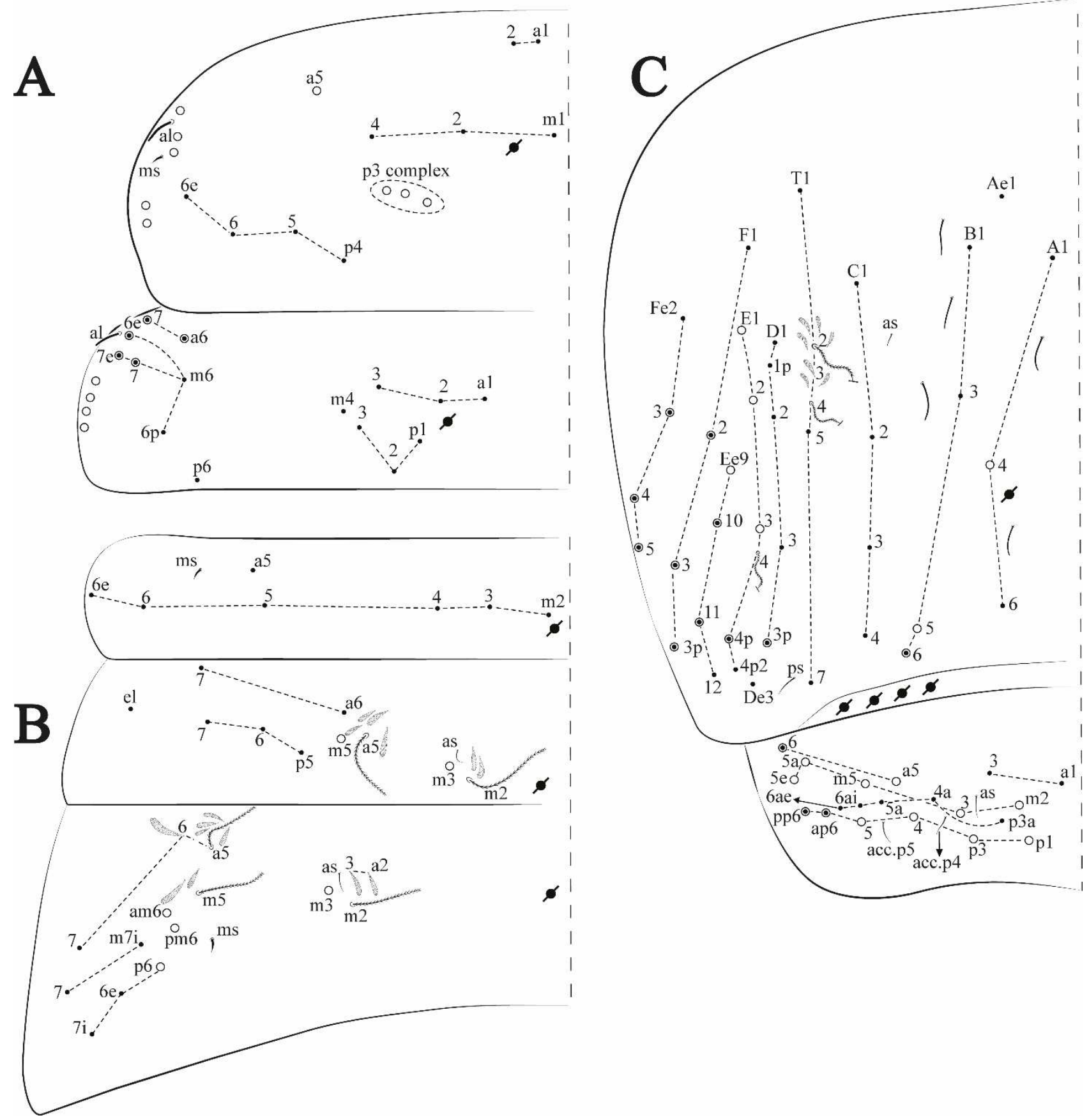

Figure 27. Trogolaphysa mariecurieae sp. nov.: Dorsal chaetotaxy. A) Th II-III; B) Abd I-III; C) Abd IV-V. 

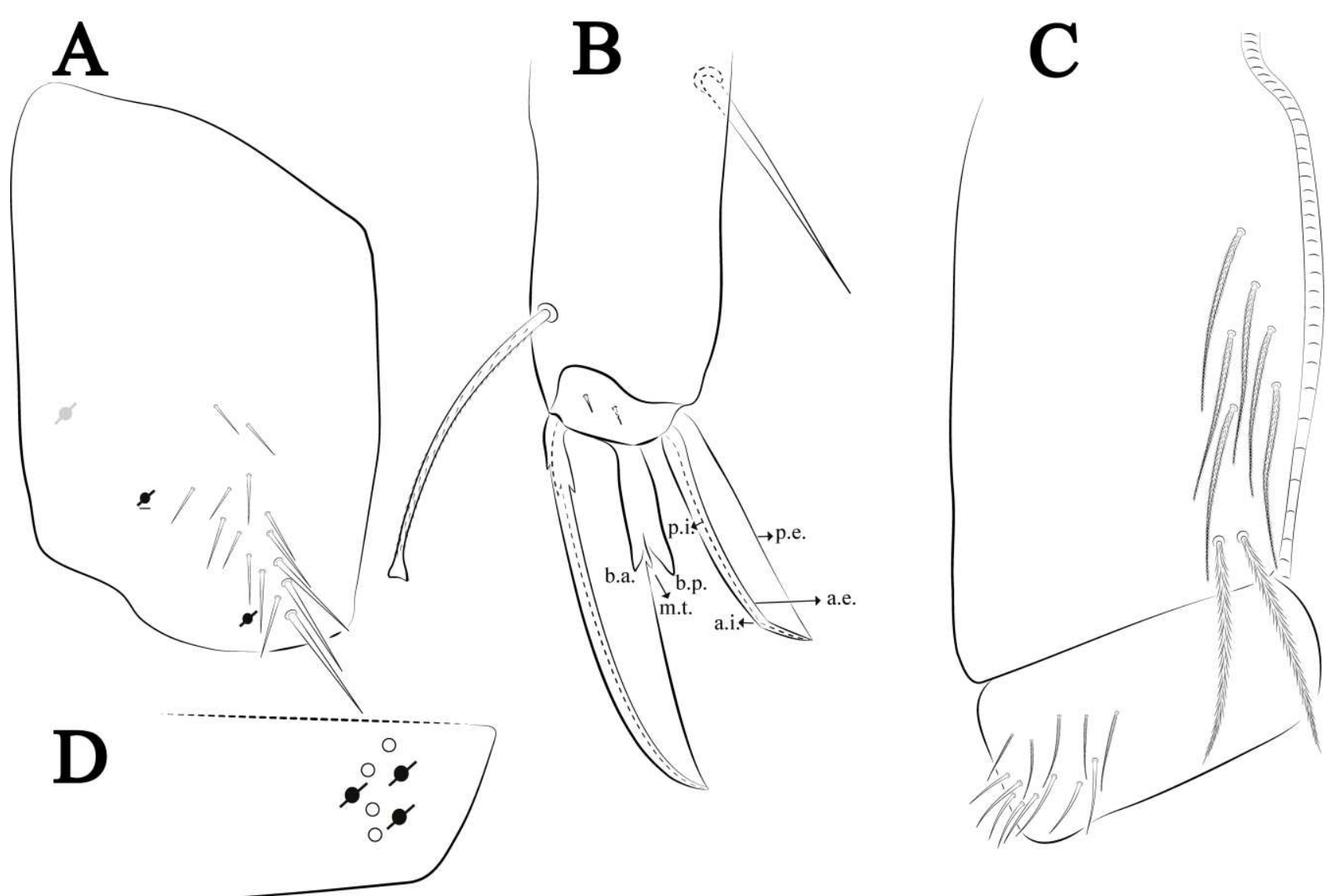

Figure 28. Trogolaphysa mariecurieae sp. nov.: A) Trochanteral organ; B) Distal tibiotarsus and empodial complex III (anterior view); C) Manubrial plate; D) Antero-lateral view of collophore chaetotaxy. 
Trogolaphysa barroca sp. nov. Brito \& Zeppelini

Figures 29-31, Tables 1-2

Type material. Holotype female in slide (13167/CRFS-UEPB): Brazil, Minas Gerais State, Mariana municipality, ALFA-0003 cave, 2009'06.8'S, 43²9'13.6”'W, 07-27.ii.2018, Bioespeleo team coll. Paratype in slide (13150/CRFS-UEPB): 1 female, same data as holotype, except 12.vi.2017. Paratype in slide (13158/CRFS-UEPB donated to MNJR): 1 female, same data as holotype. Paratype in slide (13197/CRFS-UEPB): 1 female, Brazil, Minas Gerais State, Mariana municipality, ALEA-0004 cave, 2009'00.0”S, 4329'11.8”W, 07.ii.2018, Bioespeleo team coll. Paratype in slide (13203/CRFS-UEPB): 1 female, Brazil, Minas Gerais State, Mariana municipality, ALEA-0002 cave, $20^{\circ} 08^{\prime} 56.5^{\prime}$ S, $43^{\circ} 29^{\prime} 09.8^{\prime \prime} \mathrm{W}, 27$. ii.2018, Bioespeleo team coll. Additional records see S1.

Description. Total length (head + trunk) of specimens 1.70-2.13 mm ( $\mathrm{n}=5)$, holotype $1.81 \mathrm{~mm}$. Head. Ratio antennae: trunk $=1: 1.27-1.60(\mathrm{n}=3)$, holotype $=1: 1.27$; Ant III shorter than Ant II; Ant segments ratio as, I: II, III, IV = 1: 1.90-2.41, 1.64-2.02, 2.69-3.64, holotype = 1: 1.90, 1.67, 2.69. Antennal chaetotaxy (no represented): Ant IV dorsally and ventrally with several short less ciliate mic and mac, and finger-shaped sens, dorsally with about four rod sens on longitudinal row, ventrally with one subapical-organ and several wrinkly sens (Fig. 3A); Ant III dorsally and ventrally with several short less ciliate mic and mac, and finger-shaped sens, dorsally without modified sens, ventrally with one apical psp, about nine wrinkly sens on external longitudinal row, apical organ with one finger-shaped sens, two coffee bean-like sens, and one rod sens (Fig. 3A); Ant II dorsally and ventrally with several short less ciliate mic and mac, dorsally with two sub-apical finger-shaped sens and two subapical rod sens, ventrally with one apical psp, and several wrinkly sens on longitudinal external row (Fig. 3A); and Ant I dorsally and ventrally with several short less ciliate mic and mac, dorsally with three basal spine-like sens, ventrally with about five basal spinelike sens, about five smooth mic and several finger-shaped sens (Fig. 3A). Eyes 0+0. Head dorsal chaetotaxy (Fig. 29A) with 14-15 An (An1a-3), six A (A0-5), five M (M1-5), six S (S1-6), two Ps (Ps2, Ps5), four Pa (Pa1-3, Pa5), two Pm (Pm1, Pm3), seven Pp (Pp1-7), and two Pe (Pe4, Pe6) chaetae; Pm3 as mic, A3 as mes, An1a-3, A0, A2 and Pa5 as mac; interocular p mic present. Basomedian and basolateral labial fields with a1-5 smooth, M, Me, E and L1-2 ciliate, $\mathbf{r}$ reduced (Fig. 29B). Ventral chaetotaxy with 33 ciliate chaetae and one reduced lateral spine; postlabial G14; X, X4; H1-4; J1-2, chaetae b.c. present and a collar row of five to six mes chaetae distally (Fig. 29B). Prelabral chaetae weakly ciliate. Labral chaetae smooth, no modifications. Labial papilla $\mathbf{E}$ with l.p. finger-shaped and subequal the base of apical appendage. Labial proximal chaetae smooth (an1-3, p2-3), and subequal in length (Fig. 29B). Maxillary palp with t.a. smooth and $1.14 \times$ larger than b.c.

Thorax dorsal chaetotaxy (Fig. 30A). Th II a, m, p series with two mic (a1-2), one mac (a5), three mic (m1-2, m4) and four mic (p4-6e), p3 complex with five mac, respectively, al and ms present. Th III a, m, p series with three mic (a1-3), two mes (a6-7), two mic (m4, m6p), four mes (m6-6e, $\mathbf{m} 7-7 \mathbf{e})$, and four mic (p1-3, p6), respectively. Ratio Th II: III = 1.11-1.35: $1(\mathrm{n}=5)$, holotype = 1.29: 1 .

Abdomen dorsal chaetotaxy (Figs. 30B-C). Abd I a, m series with one (a5) and six (m2-6e) mic, respectively, ms present. Abd II a, m, p series with two mic (a6-7), two mac (m3, m5), three mic (p5-7) respectively, el mic and as present; $\mathbf{a 5}$ and $\mathbf{m} \mathbf{2}$ bothriotricha surrounded by four and three fan-shaped chaetae, respectively. Abd III a, m, p series with one mic (a7), three fan-shaped chaetae (a2-3, a6), two mic (m7i-7), three mac (m3, am6, pm6), three mic (p6e, p7i-7), one mac (p6) chaetae, respectively; $\mathbf{a 5}, \mathbf{m} \mathbf{2}$ and $\mathbf{m 5}$ bothriotricha with six, two and three fan-shaped chaetae, 
respectively; as sens elongated, ms present. Abd IV A-Fe series with four mic (A1, A5-6, Ae1), one mac (A3), one mic (B1), one mes (B6), two mac (B4-5), four mic (C1-4), three mic (T1, T56), one mes (T7), five mic (D1-3, De3), one mes (D3p), one mic (E4p2), one mes (E4p), three mac (E1-3), one mic (Ee12), three mes (Ee9-11), one mic (F1), three mes (F2-3p), one mic (Fe2), three mes (Fe3-5) chaetae, respectively; T2, T4 and E4 bothriotricha surrounded by four and two (T3) fan-shaped chaetae, respectively; ps and as present, and at least seven supernumerary sens with uncertain homology ' $s$ ' (Fig. 7A); Abd. IV posteriorly with four to six psp. Abd V a, m, p series with two mic (a1, a3), one mes (a6), one mac (a5), two mes (m5a-5e), three mac (m2-3, m5), five mic (p3a-6ae), one mic (p6e), two mes (ap6, pp6), four mac (p1, p3-5) chaetae, respectively; as and acc.p4-5 present. Ratio Abd III: IV =1:3.38-5.55 ( $\mathrm{n}=5)$, holotype = 1: 5.27 . Legs. Trochanteral organ diamond shape with about 16-21 spine-like chaetae, plus 2-3 psp one external, and two (one of them present or absent) on top of posterior spines row of Omt (Fig. 31A). Unguis outer side with one paired tooth straight and not developed on proximal third; inner lamella wide with two teeth, basal pair subequal; b.p. little larger than b.a., m.t. and a.t. absent. Unguiculus with all lamellae smooth and lanceolate (a.i., a.e., p.i., p.e.) (Fig. 31B); ratio unguis: unguiculus = 1.53-1.67: $1(\mathrm{n}=5)$, holotype $=1.61: 1$. Tibiotarsal smooth chaetae about $0.61 \times$ smaller than unguiculus; tenent hair acuminate and about $0.4 \times$ smaller than unguis outer lamella.

Collophore (Fig. 31C). Anterior side with eight ciliate, apically acuminate chaetae, four proximal (thinner), one subdistal and three distal mac; lateral flap with 10 chaetae, five ciliate in the proximal row and five smooth in the distal row.

Furcula. Covered with ciliate chaetae, spine-like chaetae and scales. Manubrial plate with five ciliate chaetae (three inner mac) and three psp (Fig. 31D). Dens posterior face with two or more longitudinal rows of spines-like chaetae about 22 external and 37-39 internal, external spines larger and thinner than internal ones. Mucro with four teeth, ratio width: length $=0.33$ (holotype).

Etymology. Refers to the Baroque art (which is "barroco" noun, in Portuguese) of Mariana, Minas Gerais, type locality.

Remarks. Trogolaphysa barroca sp. nov. resembles T. formosensis by head Pm3 mic (mac in $T$. piracurucaensis, T. gisbertae sp. nov. and T. dandarae sp. nov.; mes in T. ernesti, T. sotoadamesi sp. nov. and $T$. mariecurieae sp. nov.); $3+3$ head dorsal mac like $T$. ernesti, although in the new species it is as A0, A2 and Pa5, and in T. ernesti is A0, A2-3; unguis m.t. and a.t. teeth absent like T. sotoadamesi sp. nov. and T. dandarae sp. nov. (present in T. bellini sp. nov., T. lacerta sp. nov. and T. chapelensis sp. nov.). 


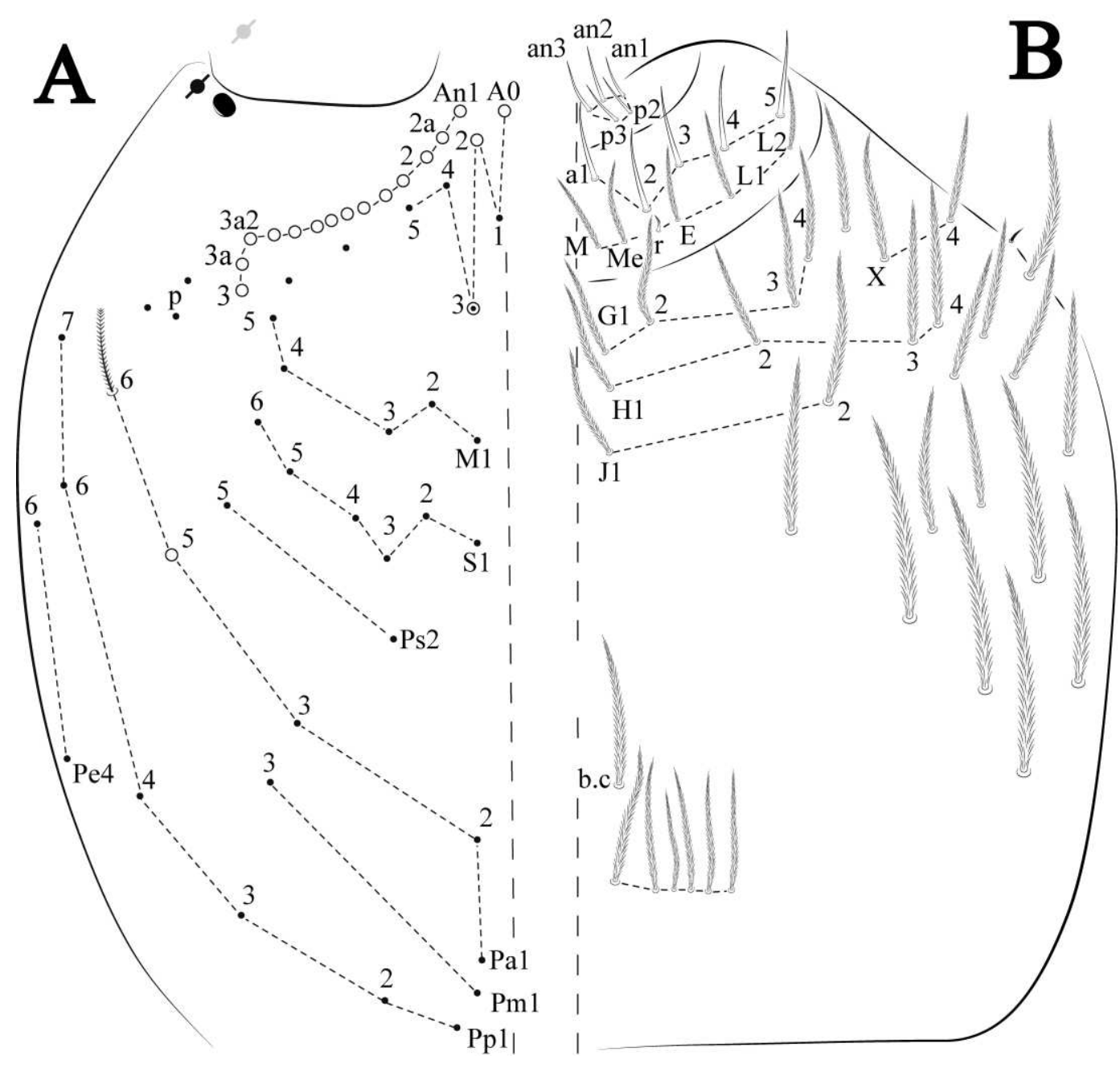

Figure 29. Trogolaphysa barroca sp. nov.: A) Head dorsal chaetotaxy; B) labial proximal chaetae, basomedial and basolateral labial fields and postlabial chaetotaxy. Black cut circle, pseudopore; Gray cut circle pseudopore at the under surface. 


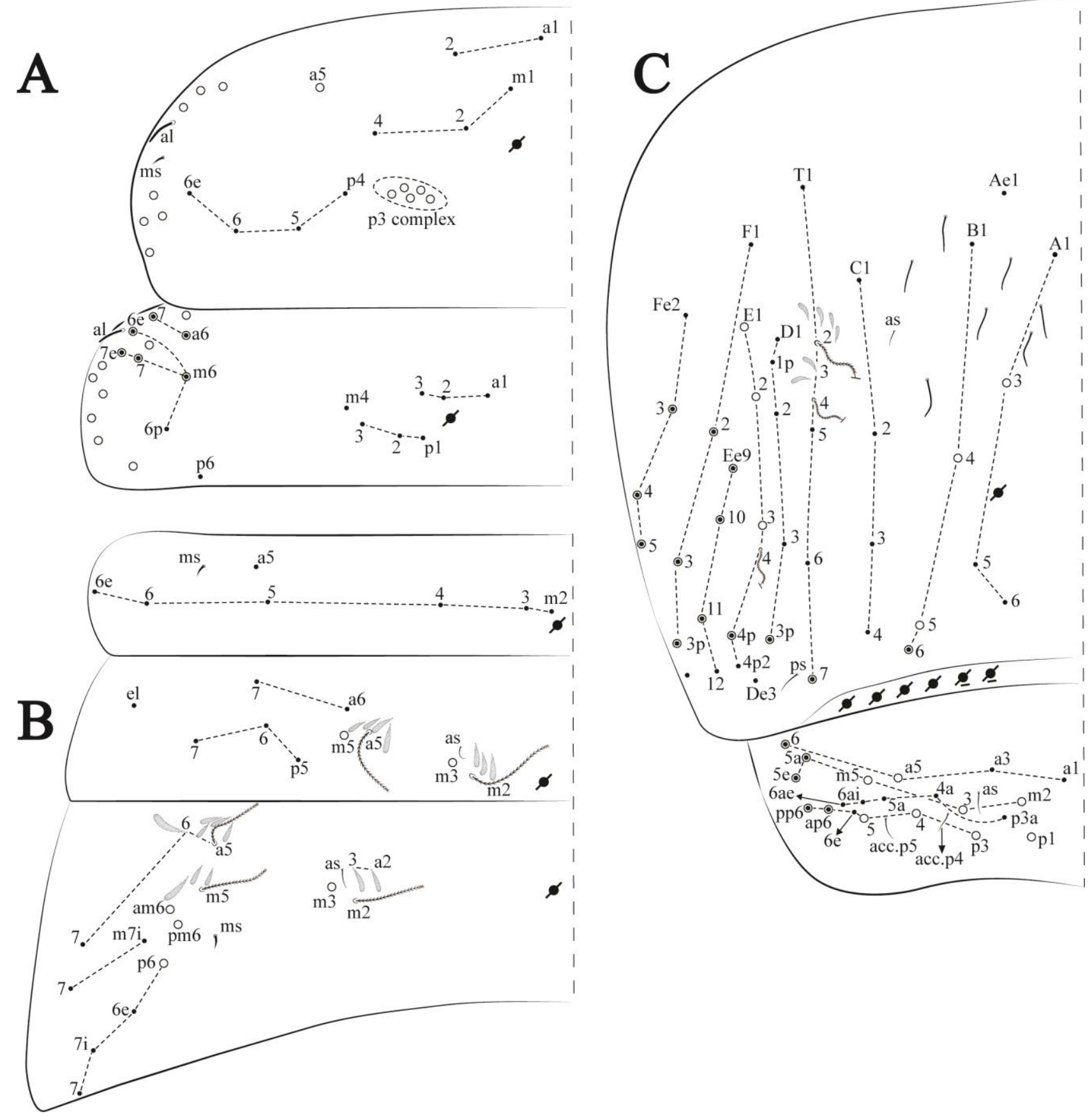

Figure 30. Trogolaphysa barroca sp. nov.: Dorsal chaetotaxy. A) Th II-III; B) Abd I-III; C) Abd IV-V. 

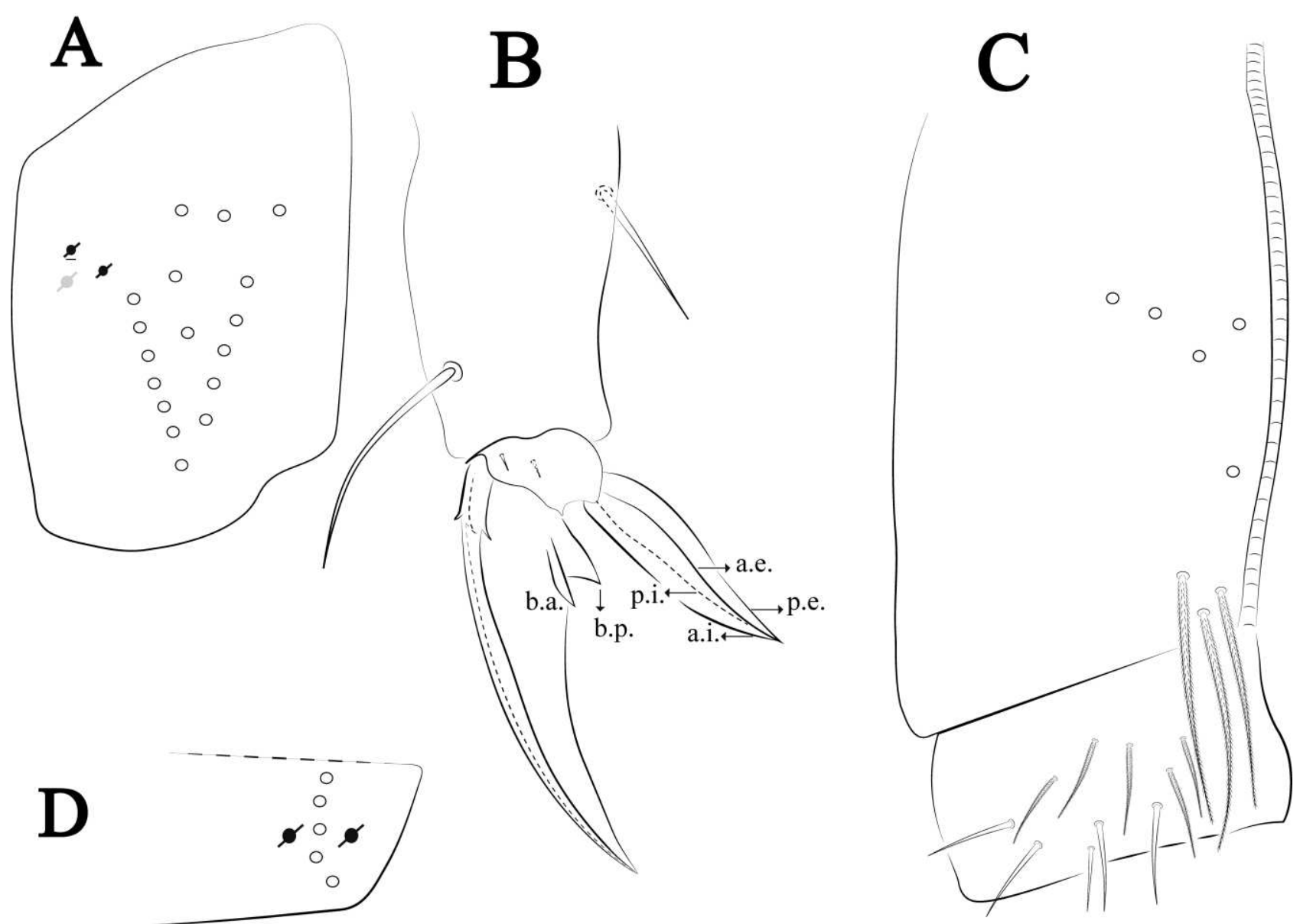

Figure 31. Trogolaphysa barroca sp. nov.: A) Trochanteral organ; B) Distal tibiotarsus and empodial complex III (anterior view); C) Manubrial plate; D) Antero-lateral view of collophore chaetotaxy. 
Trogolaphysa epitychia sp. nov. Oliveira, Lima \& Zeppelini

Figures 32-34, Tables 1-2

Type material. Holotype male in slide (10578/CRFS-UEPB): Brazil, Minas Gerais State, Conceição do Mato Dentro municipality, cave CSS-0118, next to "São Sebastião do Bom Sucesso", 1856'14.1"S, 4324'43.8”'W, 21.xi-15.xii.2016, Carste team coll. Paratypes in slides (10580, 10585/CRFS-UEPB): 2 females, same data as holotype. Paratypes in slides (10653, 10692/CRFSUEPB donated to MNJR): 1 female and 1 male, same data as holotype, except 22.xi-15.xii.2016 and 31.v-12.vi.2016, respectively. Additional records see S1.

Description. Total length (head + trunk) $1.13-1.35 \mathrm{~mm}(\mathrm{n}=5)$, holotype $1.13 \mathrm{~mm}$. Head. Ratio antennae: trunk $=1: 1.29-1.95(\mathrm{n}=5)$, holotype $=1: 1.95$; Ant III shorter than Ant II; Ant segments ratio as I: II, III, IV = 1: 1.69-2.20, 1.14-1.86, 2.37-3.52, holotype = 1: 1.71, 1.14, 2.37. Antennal chaetotaxy (no represented): Ant IV dorsally and ventrally with several short ciliate mic and mac, and finger-shaped sens, dorsally with one longitudinal row with about six rod sens, ventrally with one subapical-organ and one longitudinal row with about four wrinkly sens (Fig. 3A); Ant III dorsally and ventrally with several short ciliate mic and mac, and finger-shaped sens, dorsally without modified sens, ventrally with one apical psp, about three wrinkly sens on external longitudinal row, apical organ with two coffee bean-like sens, one rod sens and one smooth mic (Fig. 3A); Ant II dorsally and ventrally with several short ciliate mic and mac, dorsally with about six sub-apical finger-shaped sens and one wrinkly sens, ventrally with one apical psp, about three wrinkly sens on longitudinal external row (Fig. 3A); and Ant I dorsally and ventrally with several short ciliate mic and mac, three basal spine-like sens, ventrally with four basal spine-like sens, about three smooth mic, several finger-shaped sens, and two wrinkly sens (Fig. 3A). Eyes 0+0. Head dorsal chaetotaxy (Fig. 32A) with 12 An (An1a-3), six A (A0-5), four M (M1-4), five S (S2-6), two Ps (Ps2, Ps5), four Pa (Pa1-5), two Pm (Pm1, Pm3), seven Pp (Pp1-7), and two Pe (Pe4, Pe6) chaetae; Pm3 and Pa5 as mes, An1a-3a, A0 and A2 as mac; interocular p mes present. Basomedian and basolateral labial fields with a1-5 smooth, M, Me, E and L1-2 ciliate, $\mathbf{r}$ reduced (Fig. 32B). Ventral chaetotaxy with 31-32 ciliate chaetae and one reduced lateral spine; postlabial G1 -4; X, X4; H1-4; J1-2, chaetae b.c. present and a collar row of five to six mes chaetae distally (Fig. 32B). Prelabral chaetae ciliate. Labral chaetae smooth, no modifications. Labial papilla $\mathbf{E}$ with l.p. finger-shaped and surpassing the base of apical appendage. Labial proximal chaetae smooth (an1-3, p2-3) and subequal in length (Fig. 32B). Maxillary palp with t.a. smooth and $1.26 \times$ larger than b.c.

Thorax dorsal chaetotaxy (Fig. 33A). Th II a, m, p series with two mic (a1-2), one mac (a5), three mic (m1-2, m4) and four mic (p4-6e), p3 complex with three mac, respectively, al and ms present. Th III a, m, p series with three mic (a1-3), two mes (a6-a7), three mic (m4, m6-6p), three mes $(\mathbf{m 6 e}, \mathbf{m} 7-7 \mathbf{e})$, four mic $(\mathbf{p 1 - 3}, \mathbf{p 6})$ respectively. Ratio Th II: III = 1.05-1.21: $1(\mathrm{n}=5)$, holotype $=$ 1.18: 1 .

Abdomen dorsal chaetotaxy (Figs. 33B-C). Abd I a, m series with one (a5) and six (m2-6e) mic respectively, ms present. Abd II a, m, p series with two mic (a6-7), two mac (m3, m5), three mic (p5-7) respectively, el mic and as present; $\mathbf{a 5}$ and $\mathbf{m} 2$ bothriotricha surrounded by four and two fanshaped chaetae respectively. Abd III a, m, p series with two mic (a7i-7), three fan-shaped chaetae (a2-3, a6), two mic (m7i-7), three mac (m3, am6, pm6), three mic (p6e, p7i-7), one mac (p6) chaetae, respectively; $\mathbf{a 5}, \mathbf{m} \mathbf{2}$ and $\mathbf{m 5}$ bothriotricha with five, two and one fan-shaped chaetae, respectively; as sens elongated, ms present. Abd IV A-Fe series with three mic (A1, A6, Ae1), two mac (A3, A5), two mic (B1, B4), one mes (B6), one mac (B5), four mic $(\mathbf{C 1}-4)$, four mic (T1, T3, 
T5-6), one mac (T7), six mic (D1-3p, De3), two mic (E4p-4p2), three mac (E1-3), one mic (Ee11), three mes (Ee9-10, Ee12), one mic (F1), three mes (F2-3p), one mic (Fe2), three mes (Fe3-5) chaetae, respectively; T2, T4 and E4 bothriotricha surrounded by five and two fan-shaped chaetae, respectively; ps and as present, and at least seven supernumerary sens with uncertain homology ' $s$ ' (Fig. 7A); Abd. IV posteriorly with three psp. Abd V a, m, p series with three mic (a1, a3, a6), one mac (a5), two mic (m3, me5), three mac (m2, m5-5a), two mic (p3a-4a), one mes (p5a) two mac (p6ai-6ae), four mes (p5-pp6), three mac (p1, p3-4) chaetae, respectively; as, acc.p4-5 present. Ratio Abd III: IV = 1: 4.69-5.55 $(\mathrm{n}=5)$, holotype $=1: 4.88$.

Legs. Trochanteral organ in V-shape with about 15 spine-like chaetae, plus 4 psp one external, one on distal vertex and another two on top of posterior spines row of Omt (Fig. 34A). Unguis outer side with one paired tooth straight and not developed on proximal third; inner lamella wide with four teeth, basal pair subequal, b.p. little larger, not reaching the m.t. apex, m.t. just after the distal half, a.t. absent. Unguiculus with all lamellae smooth and slightly truncate (a.i., a.e., p.i., p.e.) (Fig. 34B); ratio unguis: unguiculus $=1.17-1.98: 1(\mathrm{n}=5)$, holotype $=1.17: 1$. Tibiotarsal smooth chaetae about $0.8 \times$ smaller than unguiculus; tenent hair acuminate and about $0.53 \times$ smaller than unguis outer lamella.

Collophore (Fig. 34C). Anterior side with 9 ciliate, apically acuminate chaetae, five proximal, two subdistal and two distal mac; lateral flap with 10 chaetae, five ciliate in the proximal row and five smooth in the distal row.

Furcula. Covered with ciliate chaetae, spine-like chaetae and scales. Manubrial plate with five ciliate chaetae (two inner mac) and three psp (Fig. 34D). Dens posterior face with two or more longitudinal rows of spine-like chaetae about 60 external and 34 internal, external spines larger and thinner than internal ones. Mucro with four teeth, ratio width: length $=0.30$ (holotype).

Etymology. Epitychia from Greek means success, in allusion to the collection site where the species was found São Sebastião do Bom Sucesso.

Remarks. Trogolaphysa epitychia sp. nov. resembles T. bellinii sp. nov., T. bessoni, and $T$. mariecurieae sp. nov. by the absence of eyes (T. bellinii sp. nov. rarely with $2+2$ eyes), Th II with 3+3 mac, and Th III without mac. Differentiates from T. bellinii sp. nov. and T. mariecurieae sp. nov. by Abd IV with 3+3 (A3, A5, B5), 4+4, and 2+2 mac on Abd IV respectively; on T. bellinii sp. nov. and can be distinguished from $T$. bessoni by the absence of unpaired tooth on inner lamella of unguis, external row of dens with 25 spines, inner row of dens with 20 spines (T. epitychia $\mathbf{\text { sp. nov. }}$ with one unpaired tooth m.t. on inner lamella of unguis, external row of dens with about 60 spines and inner row of dens with about 34 spines). 


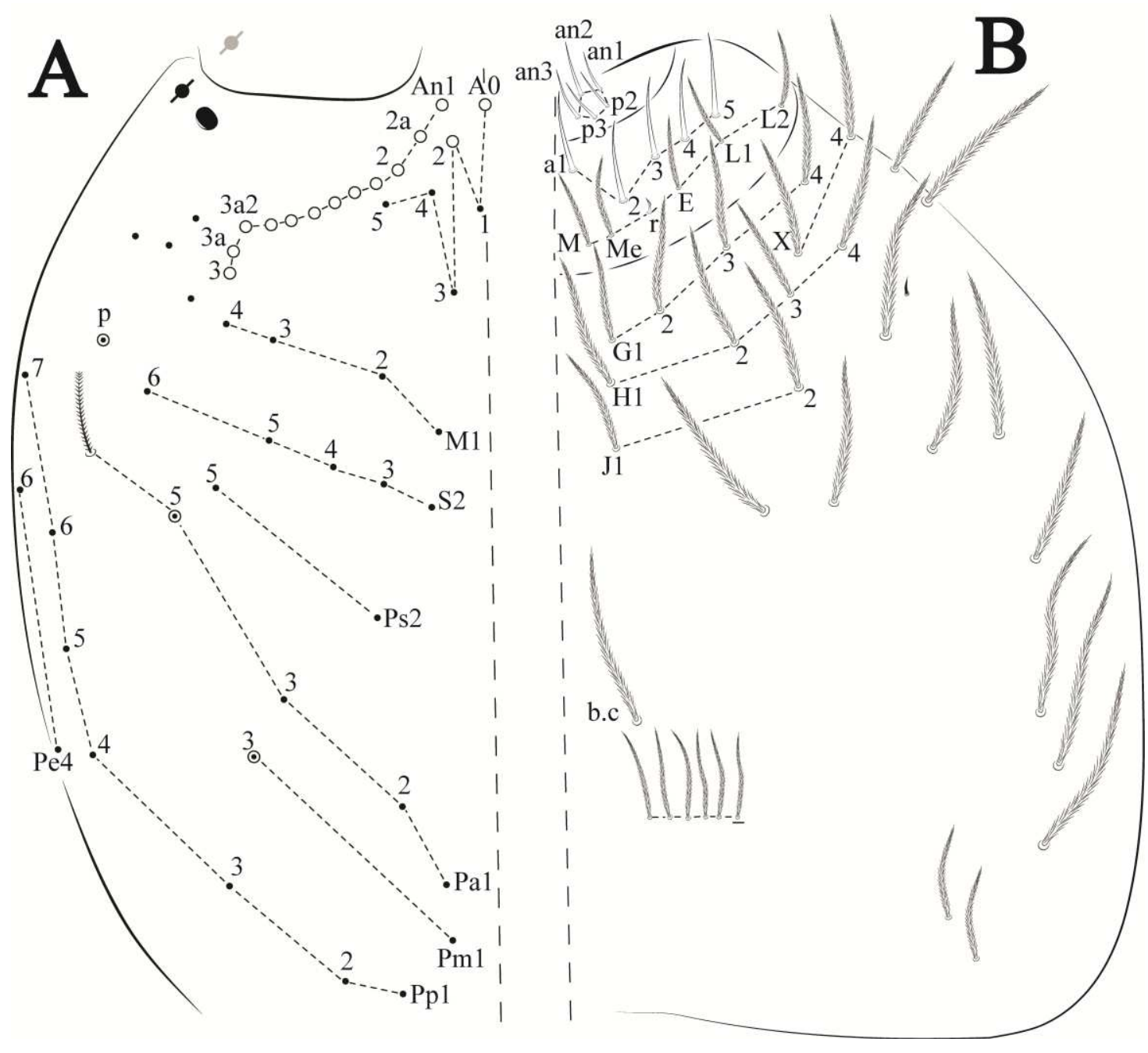

Figure 32. Trogolaphysa epitychia sp. nov.: A) Head dorsal chaetotaxy; B) labial proximal chaetae, basomedial and basolateral labial fields and postlabial chaetotaxy. Black cut circle, pseudopore; Gray cut circle pseudopore at the under surface. 


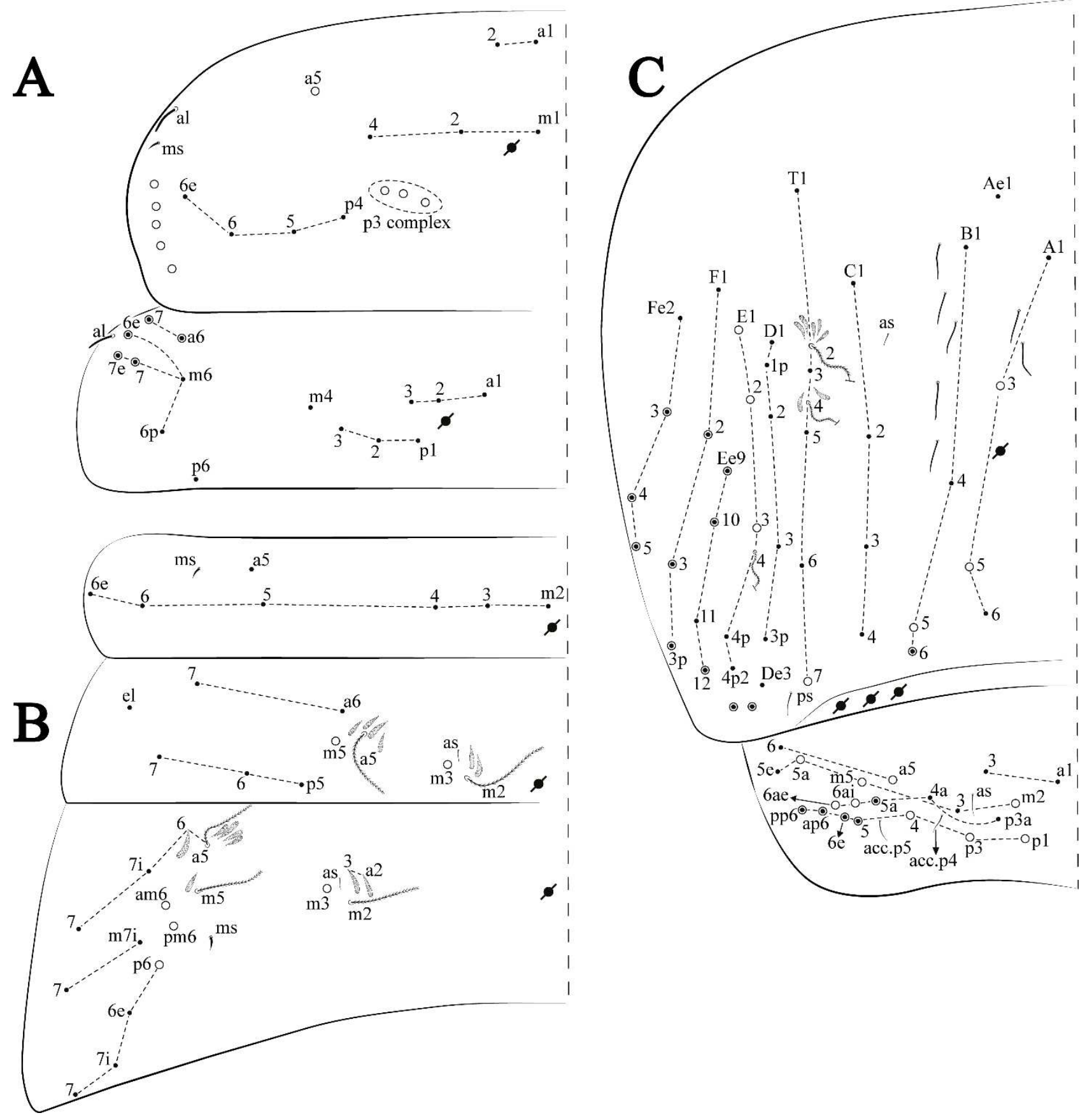

Figure 33. Trogolaphysa epitychia sp. nov.: Dorsal chaetotaxy. A) Th II-III; B) Abd I-III; C) Abd IV-V. 

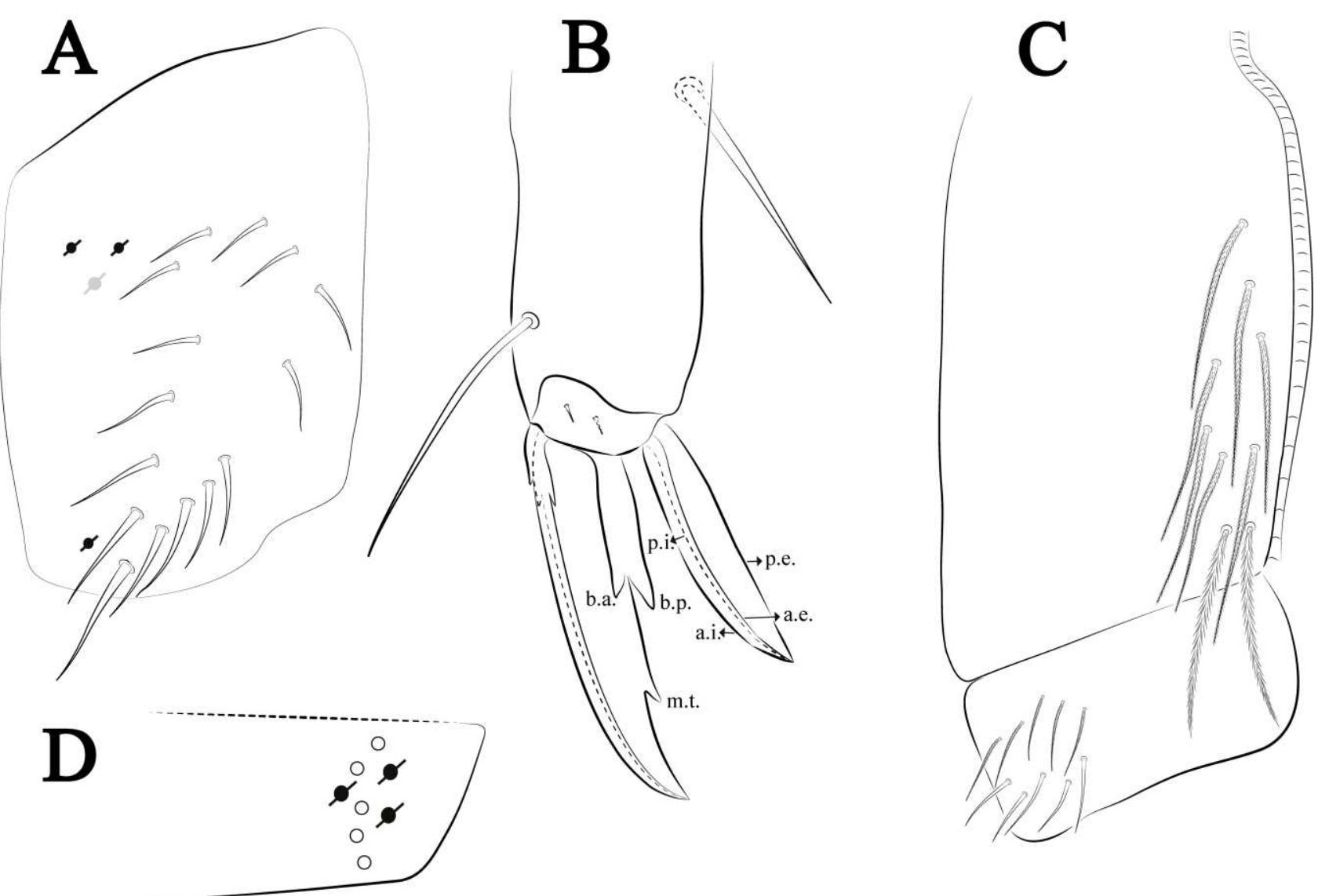

Figure 34. Trogolaphysa epitychia sp. nov.: A) Trochanteral organ; B) Distal tibiotarsus and empodial complex III (anterior view); C) Manubrial plate; D) Antero-lateral view of collophore chaetotaxy. 
Trogolaphysa zampauloi sp. nov. Lima, Oliveira \& Zeppelini

Figures 35-37, Tables 1-2

Type material. Holotype female in slide (11851/CRFS-UEPB): Brazil, São Paulo State, Ribeira municipality, cave MTD-13, nexto to "Serra Pontalhão", 24³8'47.4"S, 48 57'52.6"W, 0820.iii.2016, Carste team coll. Paratypes in slides (11875-11878/CRFS-UEPB): 2 males and 2 females, Brazil, São Paulo State, Ribeira municipality, cave MTD-02, 2437'27.3"S, 4857'35.8'W, 08-20.iii.2016. Paratype in slide (11876/CRFS-UEPB donated to MNJR). Additional records see S1.

Description. Total length (head + trunk) of specimens 1.35-1.91 mm $(\mathrm{n}=5)$, holotype $1.35 \mathrm{~mm}$. Head. Ratio antennae: trunk $=1: 1.35-1.55(\mathrm{n}=2)$, holotype $=1: 1.55$; Ant III smaller than Ant II length; Ant segments ratio as I: II, III, IV = 1: 1.71-2.38, 1.60-1.88, 2.85-3.61, holotype = 1: 2.38, 1.88, 3.61. Antennal chaetotaxy (no represented): Ant IV dorsally and ventrally with several short ciliate mic and mac, and finger-shaped sens, dorsally with about three rod sens on longitudinal row, ventrally with one subapical-organ, and about three wrinkly sens (Fig. 3A); Ant III dorsally and ventrally with several short ciliate mic and mac, and finger-shaped sens, dorsally without modified sens, ventrally with one apical psp, one apical wrinkly sens, apical organ with two coffee bean-like sens, and one rod sens (Fig. 3A); Ant II dorsally and ventrally with several short ciliate mic and mac, dorsally with about three sub-apical finger-shaped sens and two apical rod sens, ventrally with one apical psp, one longitudinal external row with two subapical finger-shaped sens and two medial wrinkly sens (Fig. 3A); and Ant I dorsally and ventrally with several short ciliate mic and mac, dorsally with three basal spine-like sens, ventrally with four basal spine-like sens, about four smooth mic and several finger-shaped sens (Fig. 3A). Eyes $0+0$ to $4+4$. Head dorsal chaetotaxy (Fig. 35A) with 14 An (An1a-3), six A (A0-5), four M (M1-4), five S (S2-6), two Ps (Ps2, Ps5), four Pa (Pa1-3, Pa5), two Pm (Pm1, Pm3), seven Pp (Pp1-7), and two Pe (Pe4, Pe6) chaetae; Pe4, Pe6, Pm3 and Pa5 as mes, An1a-3a as mac, A0 and A2 as mac, A3-5 as mes; interocular p mes present. Basomedian and basolateral labial fields with a1-5 smooth, M, Me, E and L1-2 ciliate, $\mathbf{r}$ reduced (Fig. 35B). Ventral chaetotaxy with about 37 ciliate chaetae, plus one reduced lateral spine; postlabial G1-4; X, X4; H1-4; J1-2, chaetae b.c. present and a collar row of eight mes chaetae distally (Fig. 35B). Prelabral chaetae ciliate. Labral chaetae smooth, no modifications. Labial papilla $\mathbf{E}$ with l.p. finger-shaped and surpassing the base of apical appendage. Labial proximal chaetae smooth (an1-3, p2-3) and subequal in length (Fig. 35B). Maxillary palp with t.a. smooth and $1.17 \times$ smaller than b.a.

Thorax dorsal chaetotaxy (Fig. 36A). Th II a, m, p series with two mic (a1-2), one mac (a5), three mic (m1-2, m4) and four mic (p4-6e), p3 complex with five mac, respectively, al and ms present. Th III a, m, p series with three mic (a1-3), two mes (a6-a7), three mic (m4, m6-6p), three mes $(\mathbf{m 6 e}, \mathbf{m} 7-7 \mathbf{e})$, four mic $(\mathbf{p 1}-\mathbf{3}, \mathbf{p 6})$, respectively. Ratio Th II: III $=1.02-1.48: 1(\mathrm{n}=5)$, holotype $=$ 1.21: 1

Abdomen dorsal chaetotaxy (Figs. 36B-C). Abd I a, m series with one (a5) and six (m2-6e) mic respectively, ms present. Abd II a, m, p series with two mic (a6-7), two mac (m3, m5), three mic (p5-7) respectively, el as mic and as present; $\mathbf{a 5}$ and $\mathbf{m} \mathbf{2}$ bothriotricha surrounded by three and two fan-shaped chaetae respectively. Abd III a, m, p series with one mic (a7), three fan-shaped chaetae (a2-3, a6), two mic (m7i-7), three mac (m3, am6, pm6), three mic (p6e, p7i-7), one mac (p6) chaetae respectively; $\mathbf{a 5}, \mathbf{m} \mathbf{2}$ and $\mathbf{m} \mathbf{5}$ bothriotricha with five, two and three fan-shaped chaetae respectively, as sens elongated, ms present. Abd IV A-Fe series with three mic (A1, A6, Ae1), two mac (A3, A5), one mic (B1), one mes (B6), two mac (B4-5), four mic (C1-4), three mic (T1, T5- 
6), one mes (T7), five mic (D1-3, De3), one mes (D3p), one mic (E4p2), one mes (E4p), three mac (E1-3), one mic (Ee12), one mes (Ee11), two mac (Ee9-10), one mic (F1), two mes (F3-3p), one mac (F2), one mic (Fe2), two mes (Fe3, Fe5), one mac (Fe4) chaetae, respectively; T2, T4 and E4 bothriotricha surrounded by four and four (T3) fan-shaped chaetae respectively; ps and as present, and at least six supernumerary sens with uncertain homology 's'(Fig. 7A); Abd. IV posteriorly with three psp. Abd V a, m, p series with two mic (a1, a3), one mes (a6), one mac (a5), two mes (m5a, m5e), three mac (m2-3, m5), five mic (p3a-6ae), one mic (p6e) two mes (ap6-pp6), four mac (p1, p3-5) chaetae, respectively; as, acc.p4-5 present. Ratio Abd III: IV=1: 3.29-4.28 ( $\mathrm{n}=5)$, holotype $=1: 4.10$.

Legs. Trochanteral organ diamond shape with about 27 spine-like chaetae, plus 3-4 psp one external, one on distal vertex and another two (one of them present or absent) on top of posterior spines row of Omt (Fig. 37A). Unguis outer side with one paired tooth straight and not developed on proximal third; inner lamella wide with four teeth, basal pair subequal, b.p. not reaching the m.t. apex, m.t. just after the distal half, a.t. present. Unguiculus with all lamellae smooth and lanceolate (a.i., a.e., p.i., p.e.) (Fig. 37B); ratio unguis: unguiculus $=1.63-1.84(\mathrm{n}=5)$, holotype $=$ 1: 1.79. Tibiotarsal smooth chaetae about $0.8 \times$ smaller than unguiculus; tenent hair acuminate and about $0.39 \times$ smaller than unguis outer edge.

Collophore (Fig. 37C). Anterior side with five ciliate, apically acuminate chaetae, two proximal (thinner); one subdistal and two distal mac; lateral flap with 11 chaetae, five ciliate in the proximal row and six smooth in the distal row.

Furcula. Covered with ciliate chaetae, spine-like chaetae and scales. Manubrial plate with four ciliate chaetae (two inner mac) and three psp (Fig. 37D). Dens posterior face with two or more longitudinal rows of spine-like chaetae about 30 external and 23 internal, external spines larger and thinner than internal ones. Mucro with four teeth, ratio width: length $=0.29(n=5)$. Etymology. Species named after the field biologist MSc. Robson de Almeida Zampaulo for his contribution to Brazilian biospeleology.

Remarks. Trogolaphysa zampauloi sp. nov. resembles T. caripensis; T. ernesti; T. piracurucaensis by Th III without mac, and 4+4 central mac (A3, A5, B4-5) in Abd IV, but is easily distinguished from these species by the presence of Th II with $4+4$ mac in $\mathbf{p} 3$ complex $(6+6$ T. caripensis, $T$. ernesti, T. piracurucaensis). For more comparisons see remarks in T. crystallensis sp. nov. 


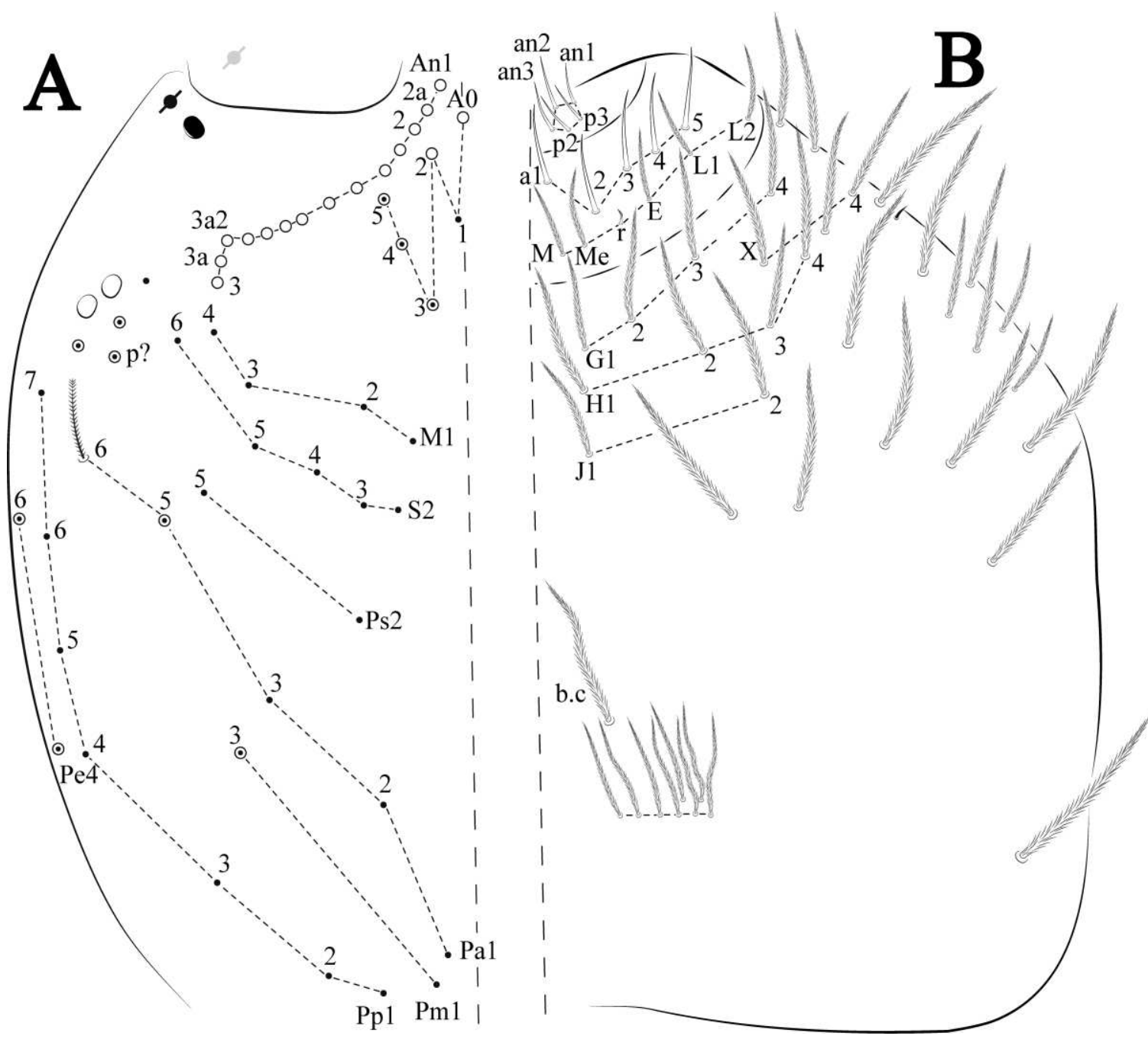

Figure 35. Trogolaphysa zampauloi sp. nov.: A) Head dorsal chaetotaxy; B) labial proximal chaetae, basomedial and basolateral labial fields and postlabial chaetotaxy. Black cut circle, pseudopore; Gray cut circle pseudopore at the under surface. 


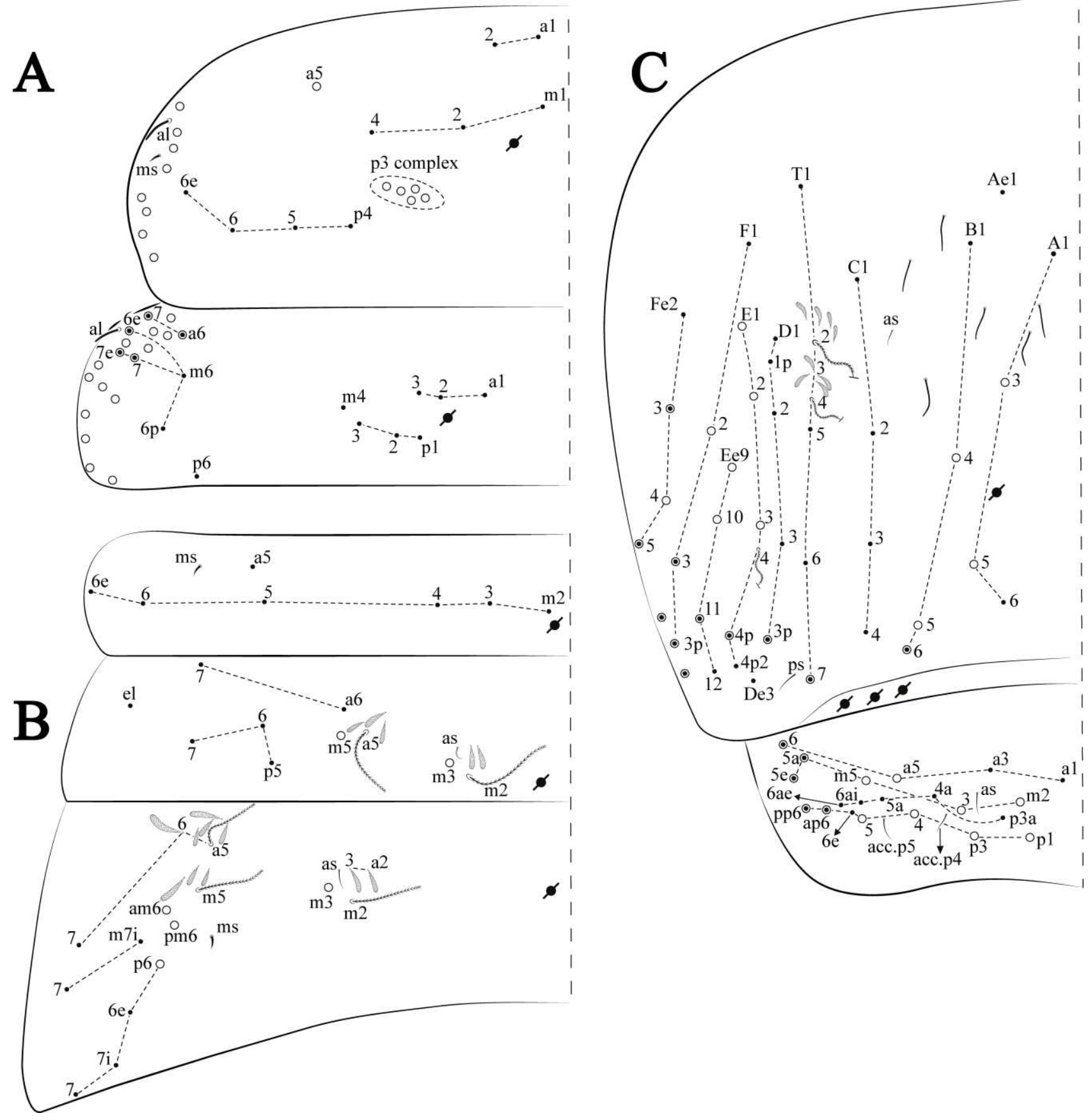

Figure 36. Trogolaphysa zampauloi sp. nov.: Dorsal chaetotaxy. A) Th II-III; B) Abd I-III; C) Abd IV-V. 

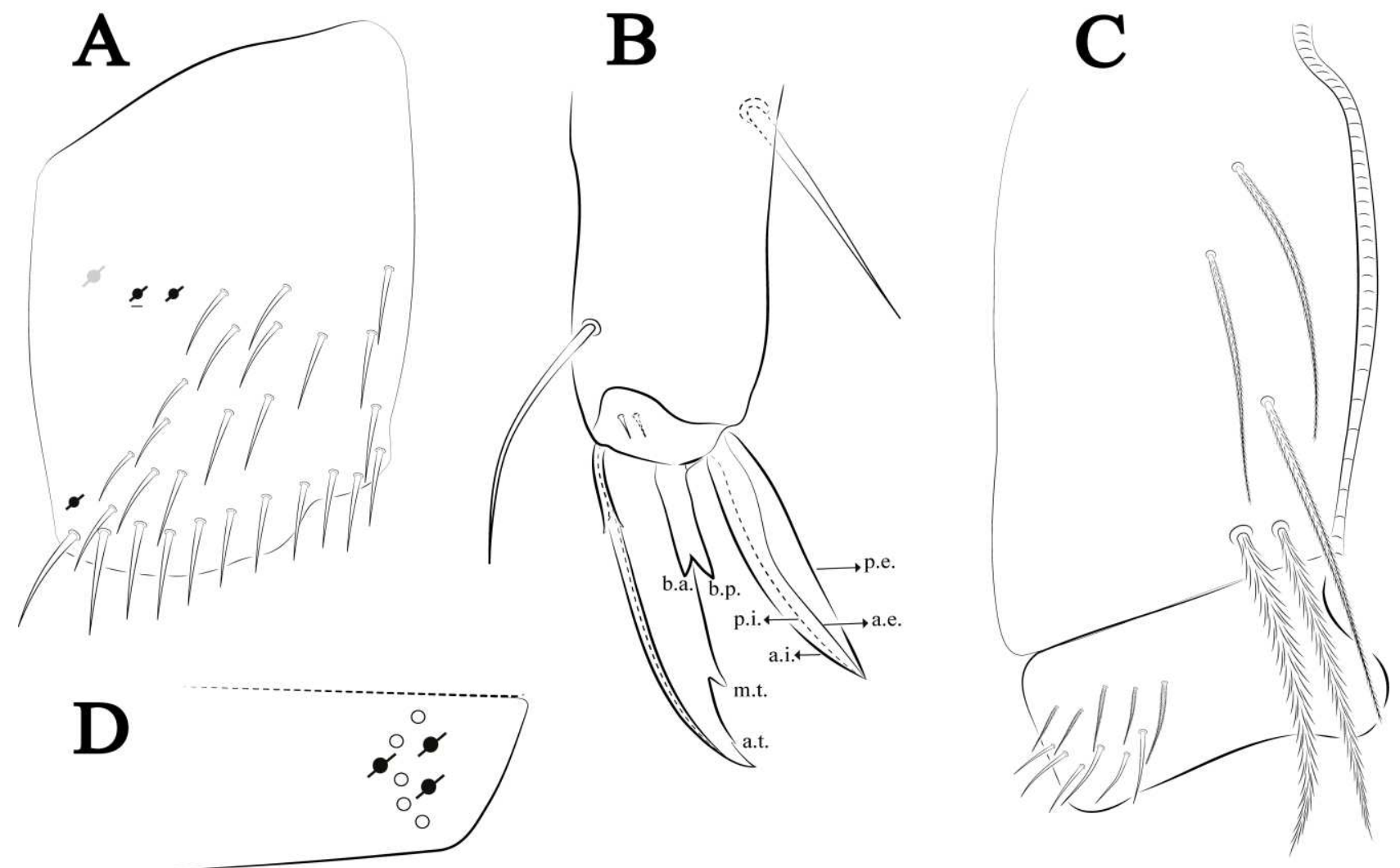

Figure 37. Trogolaphysa zampauloi sp. nov.: A) Trochanteral organ; B) Distal tibiotarsus and empodial complex III (anterior view); C) Manubrial plate; D) Antero-lateral view of collophore chaetotaxy. 
Trogolaphysa gisbertae sp. nov. Brito \& Zeppelini

Figures 38-40, Tables 1-2

Type material. Holotype female in slide (6668/CRFS-UEPB): Brazil, Pará State, Parauapebas municipality, cave N1N8-N8-017, next to “Serra Norte", 06¹0'05.9”S, 5009'25.6”'W, 0229.iv.2015, Carste team coll. Paratype in slide (6669/CRFS-UEPB donated to MNJR): 1 female, same data as holotype, except 04.ix-06.x.2014. Paratype in slide (6973/CRFS-UEPB): 1 female, same data as holotype, except 04.ix-06.x.2014. Paratypes in slides (6657, 7138/CRFS-UEPB): 2 females, Brazil, Pará State, Parauapebas municipality, N1N8-N8-020 cave, 06¹0’07.8”S, 5009’25.4’W, 17.vii-04.viii.2014, Carste team coll. Additional records see S1.

Description. Total length (head + trunk) of specimens $1.10-1.23 \mathrm{~mm}(\mathrm{n}=5)$, holotype $1.15 \mathrm{~mm}$. Head. Ratio antennae: trunk $=1: 1.44-1.55(\mathrm{n}=3)$; Ant segments ratio as I: II, III, IV=1: $1.67-$ $2.43,1.58-2.63,2.91-5.46$, holotype $=1: 2.03,-, 3.90$. Antennal chaetotaxy (no represented): Ant IV dorsally and ventrally with several short ciliate mic and mac, and finger-shaped sens, dorsally with about five rod sens in row, ventrally with one subapical-organ and several wrinkly sens row (Fig. 3A); Ant III dorsally and ventrally with several short ciliate mic and mac, and finger-shaped sens, dorsally without modified sens, ventrally with one apical psp, about four wrinkly sens on external longitudinal row, apical organ with one finger-shaped sens, two coffee bean-like sens, and one rod sens (Fig. 3A); Ant II dorsally and ventrally with several short ciliate mic and mac, dorsally with four finger-shapedd sens in row and two subapical rod sens, ventrally with one apical psp, and about five wrinkly sens on longitudinal external row (Fig. 3A); and Ant I dorsally and ventrally with several short ciliate mic and mac, dorsally with three basal spine-like sens, ventrally with four basal spine-like sens, about five smooth mic and several fniger-shaped sens (Fig. 3A). Eyes 0+0. Head dorsal chaetotaxy (Fig. 38A) with 11 An (An1a-3), six A (A0-5), four M (M1-4), five S (S1-5), two Ps (Ps2, Ps5), four Pa (Pa1-5), two Pm (Pm1, Pm3), seven Pp (Pp1-7), and two Pe (Pe4, Pe6) chaetae; An1a-3a, A0, A2-3, Pa5 and Pm3 as mac; interocular p absent. Basomedian and basolateral labial fields with a1-5 smooth, M, Me, E and L1-2 ciliate, $\mathbf{r}$ reduced (Fig. 38B). Ventral chaetotaxy with 20 ciliate chaetae and one reduced lateral spine; postlabial $\mathbf{G 1 - 4 ;} \mathbf{X}, \mathbf{X} 4$; H1-4; J1-2, chaetae b.c. present and a collar row of three to four mes chaetae distally (Fig. 38B). Prelabral chaetae ciliate. Labral chaetae smooth, no modifications. Labial papilla $\mathbf{E}$ with l.p. fingershaped and surpassing the base of apical appendage. Labial proximal chaetae smooth (an1-3, p2-3) and subequal in length (Fig. 38B). Maxillary palp with t.a. smooth and $1.32 \times$ larger than b.c. Thorax dorsal chaetotaxy (Fig. 39A). Th II a, m, p series with two mic (a1-2), one mac (a5), three mic (m1-2, m4) and four mic (p4-6e), p3 complex with five mac, respectively, al and ms presents. Th III a, m, p series with three mic (a1-3), two mes (a6-7), three mic (m4, m6-6p), three mes (m6e, m7-7e), and four mic (p1-3, p6), respectively. Ratio Th II: III = 1.00-2.60: $1(\mathrm{n}=5)$, holotype $=1.28: 1$.

Abdomen dorsal chaetotaxy (Figs. 39B-C). Abd I a, m series with one (a5) and six (m2-6e) mic, respectively, ms present. Abd II a, m, p series with two mic (a6-7), two mac (m3, m5), three mic (p5-7) respectively, el mic and as present; $\mathbf{a 5}$ and $\mathbf{m} 2$ bothriotricha surrounded by four and two fanshaped chaetae, respectively. Abd III a, m, p series with one mic (a7), three fan-shaped chaetae (a2-3, a6), two mic (m7i-7), three mac (m3, am6, pm6), three mic (p6e, p7i-7), one mac (p6) chaetae, respectively; $\mathbf{a 5}, \mathbf{m} \mathbf{2}$ and $\mathbf{m 5}$ bothriotricha with six, two and three fan-shaped chaetae, respectively, as sens elongated, ms present. Abd IV A-Fe series with four mic (A1, A5-6, Ae1), one mac (A3), one mic (B1), one mes (B6), two mac (B4-5), four mic (C1-4), four mic (T1, T5-7), five mic (D1-3, De3), one mes (D3p), one mic (E4p2), one mes (E4p), three mac (E1-3), one mic 
(Ee12), three mes (Ee9-11), one mic (F1), three mes (F2-3p), one mic (Fe2), three mes (Fe3-5) chaetae, respectively; T2, T4 and E4 bothriotricha surrounded by four and two (T3) fan-shaped chaetae, respectively; ps and as present, and at least six supernumerary sens with uncertain homology 's'(Fig. 7A); Abd. IV posteriorly with one to three psp. Abd V a, m, p series with three mic (a1, a3), one mes (a6), one mac (a5), two mes (m5a-5e), three mac (m2-3, m5), five mic (p3a-6ae), one mic (p6e), two mes (ap6, pp6), four mac (p1, p3-5) chaetae, respectively; as and acc.p4-5 present. Ratio Abd III: IV = 1: 3.29-4.90 $(\mathrm{n}=5)$, holotype = 1: 3.29 .

Legs. Trochanteral organ diamond shape with about 25 spine-like chaetae, plus 2 psp one external, and one on distal vertex of Omt (Fig. 40A). Unguis outer side with one paired tooth straight and not developed on proximal third; inner lamella wide with three teeth, basal pair subequal, b.p. not reaching the m.t. apex, m.t. just after the distal half, a.t. absent. Unguiculus with lamellae smooth and slightly truncate (a.i., a.e., p.i.), except p.e. slightly serrate (Fig. 40B); ratio unguis: unguiculus $=1.59-2.05: 1(\mathrm{n}=5)$, holotype $=1.62: 1$. Tibiotarsal smooth chaetae about $0.9 \times$ smaller than unguiculus; tenent hair acuminate and about $0.53 \times$ smaller than unguis outer lamella.

Collophore (Fig. 40C). Anterior side with five ciliate, apically acuminate chaetae, one proximal (thinner); two subdistal and two distal mac; lateral flap with 10 chaetae, five ciliate in the proximal row and five smooth in the distal row.

Furcula. Covered with ciliate chaetae, spine-like chaetae and scales. Manubrial plate with five ciliate chaetae (three inner mac) and three psp (Fig. 40D). Dens posterior face with two or more longitudinal rows of spine-like chaetae about 18 external and 24 internal, external spines larger and thinner than internal ones. Mucro with four teeth, ratio width: length $=0.26$ (holotype).

Etymology. Honor to Gisberta Salce Júnior, Brazilian woman, murdered in 2006 (Porto, Portugal) in a transphobia crime.

Remarks. Trogolaphysa gisbertae sp. nov. resembles $T$. ernesti and $T$. formosensis by $0+0$ head dorsal mac (1+1 T. piracurucaensis, $T$. dandarae sp. nov. and $T$. barroca $\mathbf{s p . ~ n o v . ) ; ~} 5+5$ head dorsal mac like $T$. dandarae sp. nov., but it is easily distinguishable by Th II $\mathbf{p 3}$ complex and Th III mac ( $5+5$ and $0+0,6+6$ and $3+3$, respectively); and unguis with m.t. present (absent in T. sotoadamesi sp. nov., $T$. barroca sp. nov.). 


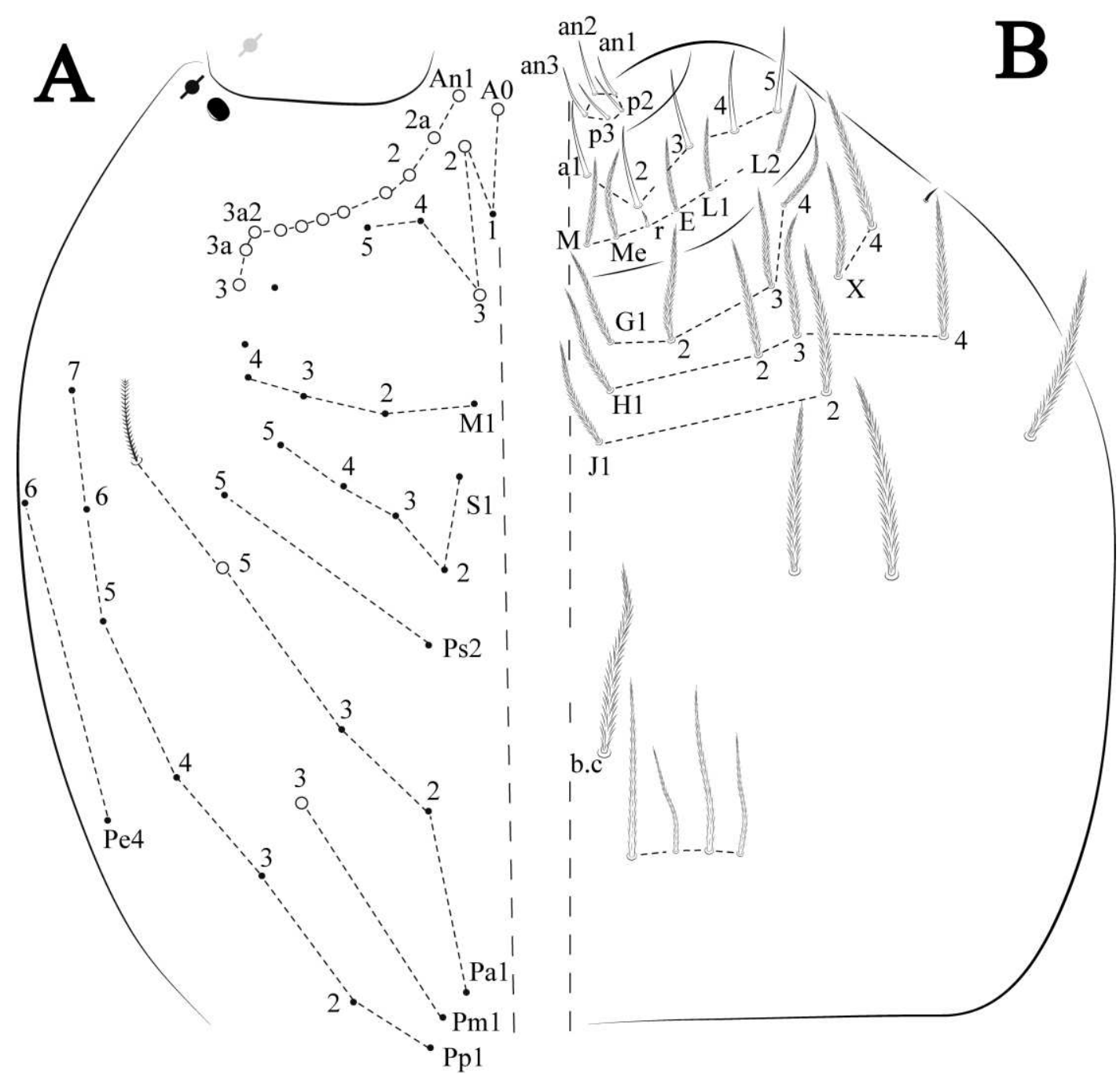

Figure 38. Trogolaphysa gisbertae sp. nov.: A) Head dorsal chaetotaxy; B) labial proximal chaetae, basomedial and basolateral labial fields and postlabial chaetotaxy. Black cut circle, pseudopore; Gray cut circle pseudopore at the under surface. 


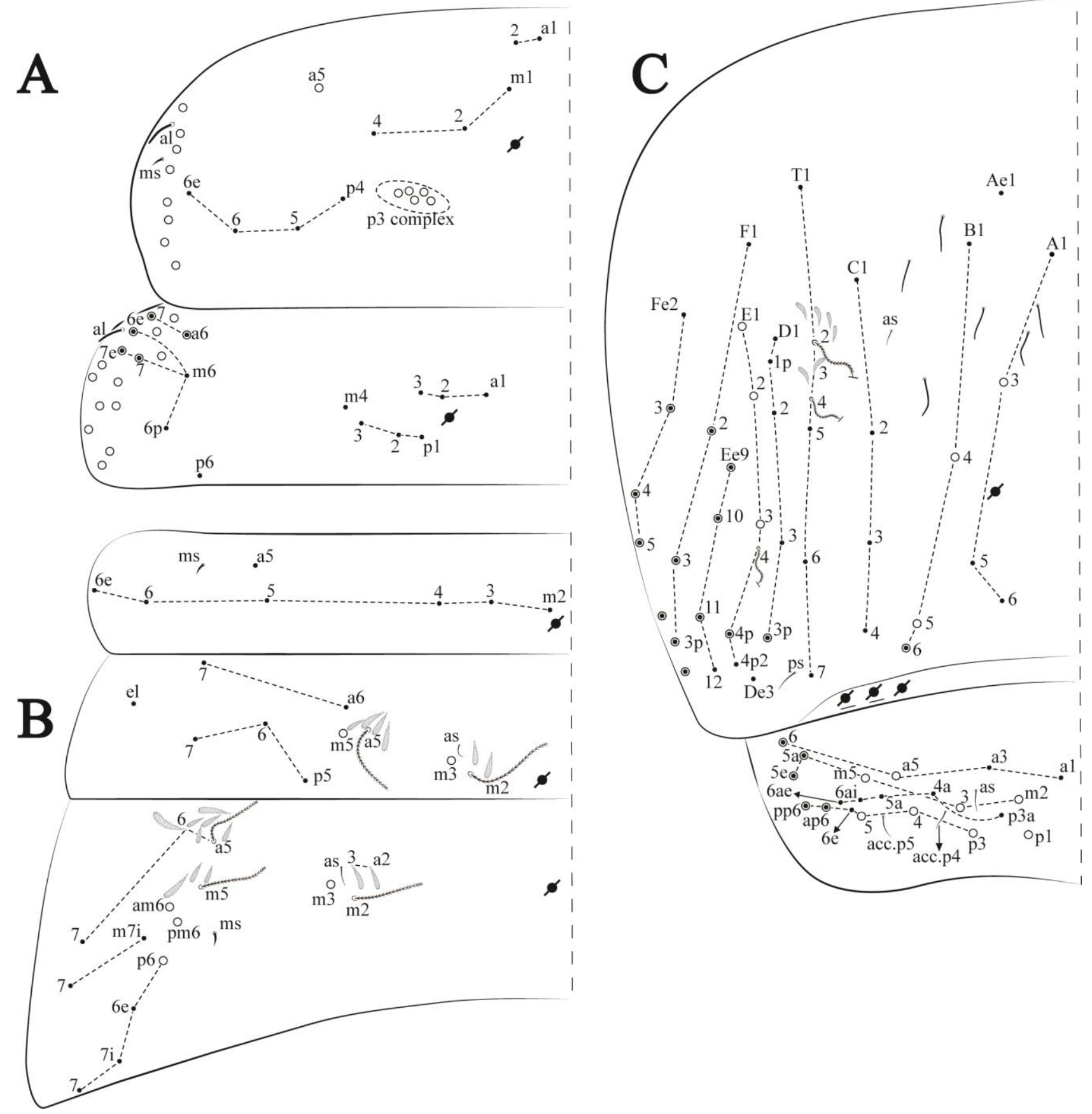

Figure 39. Trogolaphysa gisbertae sp. nov.: Dorsal chaetotaxy: A) Th II-III; B) Abd I-III; C) Abd IV-V. 

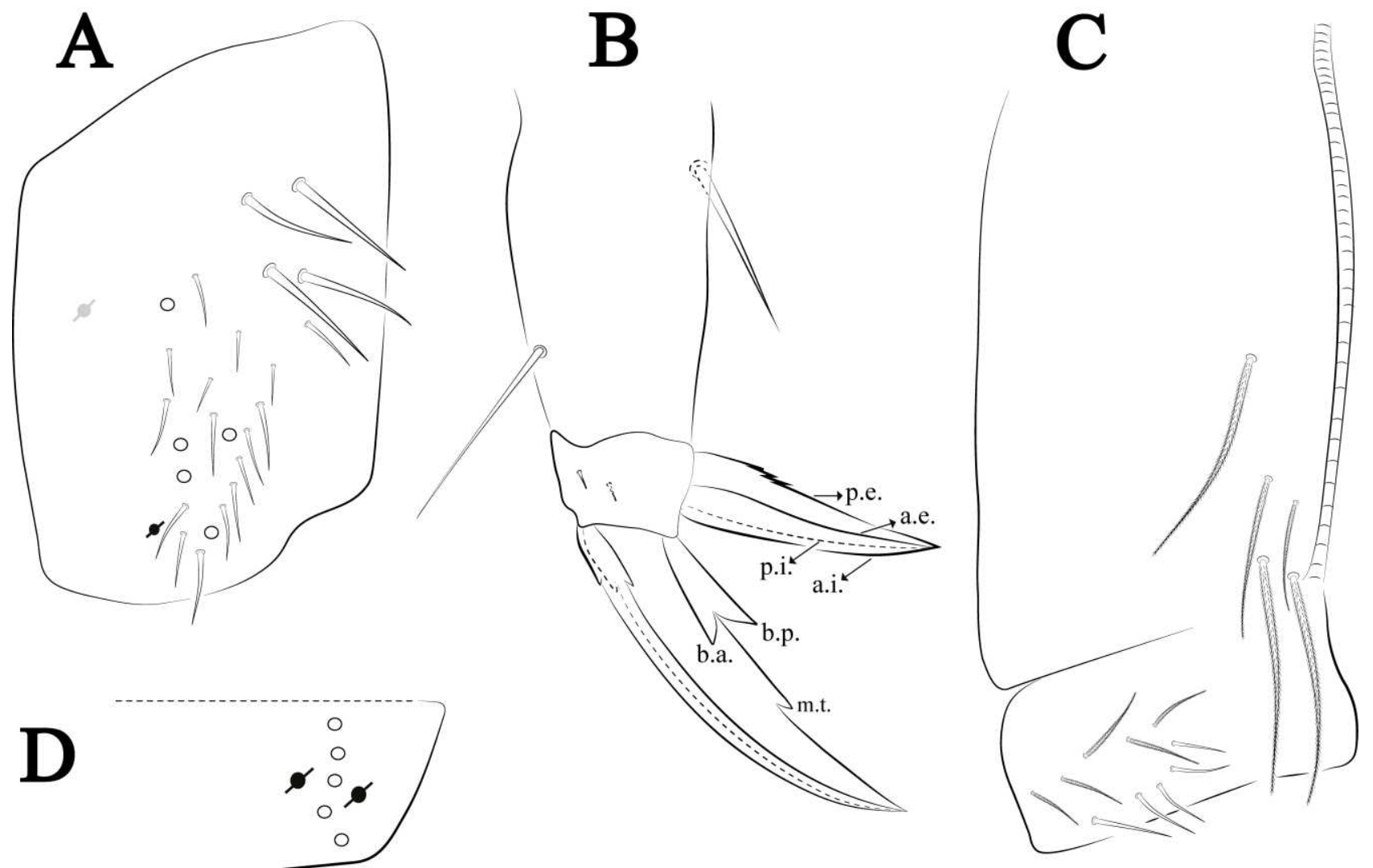

Figure 40. Trogolaphysa gisbertae sp. nov.: A) Trochanteral organ; B) Distal tibiotarsus and empodial complex III (anterior view); C) Manubrial plate; D) Antero-lateral view of collophore chaetotaxy. 
Trogolaphysa dandarae sp. nov. Brito \& Zeppelini

Figures 41-43, Tables 1-2

Type material. Holotype female in slide (12775/CRFS-UEPB): Brazil, Pará State, Parauapebas municipality, cave N4WS-0018/48, next to "Serra Norte",0604'34.5”S, 50¹1'37.7”'W, 2130.vii.2018, Brandt Meio Ambiente team coll. Paratype in slide (12776/CRFS-UEPB donated to MNJR): 1 female, same data as holotype. Paratypes in slides (12777, 12778/CRFS-UEPB): 2 females, same data as holotype. Paratypes in slides (12772, 12773/CRFS-UEPB): 2 females, Brazil, Pará State, Parauapebas municipality, N4WS-0016 cave, 0604’35.5’S, 50¹1'37.1’'W, 2130.vii.2018, Brandt Meio Ambiente team coll. Additional records see S1.

Description. Total length (head + trunk) of specimens $1.43-1.75 \mathrm{~mm}(\mathrm{n}=5)$, holotype $1.58 \mathrm{~mm}$. Head. Ratio antennae: trunk = 1: 0.83-0.98 $(\mathrm{n}=4)$, holotype $=1: 0.83$; Ant III larger than Ant II; Ant segments ratio as I: II: III: IV = 1: 1.36-1.77: 1.65-2.03: 2.84-3.27, holotype = 1: 1.72: 1.99: 3.21. Antennal chaetotaxy (no represented): Ant IV dorsally and ventrally with several short ciliate mic and mac, and finger-shaped sens, dorsally with about two rod sens sub-apical on longitudinal row, ventrally with one subapical-organ and about three wrinkly sens on longitudinal row (Fig. 3A); Ant III dorsally and ventrally with several short ciliate mic and mac, and finger-shaped sens, dorsally without modified sens, ventrally with one apical psp, about three wrinkly sens and three smooth mic on external longitudinal row, apical organ with one finger-shaped sens, two coffee bean-like sens, and one rod sens (Fig. 3A); Ant II dorsally and ventrally with several short ciliate mic and mac, dorsally with about four sub-apical finger-shaped sens and two subapical rod sens, ventrally with one apical psp, and several wrinkly sens on longitudinal external row (Fig. 3A); and Ant I dorsally and ventrally with several short ciliate mic and mac, dorsally with three basal spinelike sens, ventrally with four basal spine-like sens, about five smooth mic and several finger-shaped sens (Fig. 3A). Eyes 0+0. Head dorsal chaetotaxy (Fig. 41A) with 12 An (An1a-3), six A (A0-5), four M (M1-4), six S (S1-6), two Ps (Ps2, Ps5), four Pa (Pa1-5), two Pm (Pm1, Pm3), seven Pp (Pp1-7), and two Pe (Pe4, Pe6) chaetae; A1 as mes, An1a-3, A0, A2, S5, Pa5 and Pm3 as mac; interocular p mic present. Basomedian and basolateral labial fields with a1-5 smooth, M, Me, E and L1-2 ciliate, $\mathbf{r}$ reduced (Fig. 41B). Ventral chaetotaxy with 28 ciliate chaetae and one reduced lateral spine; postlabial G1-4; X, X4; H1-4; J1-2, chaetae b.c. present and a collar row of five chaetae distally (Fig. 41B). Prelabral chaetae ciliate. Labral chaetae smooth, no modifications. Labial papilla $\mathbf{E}$ with l.p. finger-shaped and subequal the base of apical appendage. Labial proximal chaetae smooth (an1-3, p2-3) and subequal in length (Fig. 41B). Maxillary palp with t.a. smooth and $1.58 \times$ larger than b.c.

Thorax dorsal chaetotaxy (Fig. 42A). Th II a, m, p series with two mic (a1-2), one mac (a5), three mic (m1-2, m4) and four mic (p4-6e), p3 complex with six mac, respectively, al and ms presents. Th III a, m, p series with three mic (a1-3), two mes (a6-7), two mic (m6-6p), three mes (m6e, m7-7e), and one mic (p6), respectively. Ratio Th II: III = 0.82-1.13: $1(\mathrm{n}=6)$, holotype = 1.13: 1 . Abdomen dorsal chaetotaxy (Figs. 42B-C). Abd I a, m series with one (a5) and six (m2-6e) mic, respectively, ms present. Abd II a, m, p series with two mic (a6-7), two mac (m3, m5), three mic (p5-7) respectively, el mic and as present; $\mathbf{a 5}$ and $\mathbf{m} \mathbf{2}$ bothriotricha surrounded by four and four fan-shaped chaetae, respectively. Abd III a, m, p series with one mic (a7), three fan-shaped chaetae (a2-3, a6), two mic (m7i-7), three mac (m3, am6, pm6) and three mic (p6e-7), one mac (p6) chaetae respectively; $\mathbf{a 5}, \mathbf{m} \mathbf{2}$ and $\mathbf{m} \mathbf{5}$ bothriotricha with five, two and two fan-shaped chaetae, respectively, as sens elongated, ms present. Abd IV A-Fe series with four mic (A1, A5-6, Ae1), one mac (A3), one mic (B1), one mes (B6), two mac (B4-5), four mic (C1-4), three mic (T1, T5- 
6), one mes (T7), five mic (D1-3, De3), one mes (D3p), one mic (E4p2), one mes (E4p), three mac (E1-3), one mic (Ee12), three mes (Ee9-11), one mic (F1), three mes (F2-3p), one mic (Fe2), three mes (Fe3-5) chaetae, respectively; T2, T4 and $\mathbf{E 4}$ bothriotricha surrounded by four and two (T3) fan-shaped chaetae, respectively; ps and as present, and at least six supernumerary sens with uncertain homology ' $s$ ' (Fig. 7A); Abd. IV posteriorly with three psp. Abd V a, m, p series with three mic (a1, a3), one mes (a6), one mac (a5), two mic (m5a-5e), three mac (m2-3, m5), five mic (p3a-6ae), one mic (p6e), two mes (ap6, pp6), four mac (p1, p3-5) chaetae, respectively; as and acc.p4-5 present. Ratio Abd III: IV = 1: 2.98-4.82 $(\mathrm{n}=6)$, holotype $=1: 3.81$.

Legs. Trochanteral organ diamond shape with about 19 spine-like chaetae, plus 2-3 psp one external, one on distal vertex and another (present or absent) on top of posterior spines row of Omt (Fig. 43A). Unguis outer side with one paired tooth straight and not developed on proximal third; inner lamella wide with two teeth, basal pair subequal, m.t. and a.t. absent. Unguiculus with all lamellae smooth and lanceolate (a.i., a.e., p.i., p.e.) (Fig. 43B); ratio unguis: unguiculus $=1.49$ 1.80: $1(\mathrm{n}=6)$, holotype $=1.80: 1$. Tibiotarsal smooth chaetae about $1.25 \times$ smaller than unguiculus; tenent hair slightly capitate and about $0.54 \times$ smaller than unguis outer lamella.

Collophore (Fig. 43C). Anterior side with 11 ciliate, apically acuminate chaetae, six proximal (thinner); two subdistal and three distal mac; lateral flap with 11 chaetae, five ciliate in the proximal row and six smooth in the distal row.

Furcula. Covered with ciliate chaetae, spine-like chaetae and scales. Manubrial plate with four ciliate chaetae (two inner mac) and three psp (Fig. 43D). Dens posterior face with two or more longitudinal rows of spine-like chaetae about 31-39 external and 18-21 internal, external spines larger and thinner than internal ones. Mucro with three teeth (Fig. 43E), ratio width: length $=0,28$ (holotype).

Etymology. Honor to Dandara Kettley, Brazilian man, transvestite, murdered in 2017 (Ceará, Brazil) in a homophobia crime.

Remarks. Trogolaphysa dandarae sp. nov. resembles $T$. ernesti, $T$. formosensis and $T$. piracurucaensis by chaetae head S5 mac (all other Brazilian cave species with S5 mic); head Pm3 mac as in T. gisbertae sp. nov., but they are different in terms of head ventral proximal collar mac, unguiculus, tenent hair and collophore anterior distal chaetae $(5+5$, smooth pe, capitate, $3+3$ and $4+4$, serrate pe, acuminate, $2+2$, respectively); Th II P3 complex with $6+6$ and Th III with $3+3$ mac (6+6 and $0+0$ in T. lacerta sp. nov., T. piracurucaensis, T. ernesti and T. caripensis); T. dandarae sp. nov., T. belizeana and T. jacobyi are the only cave species with $3+3$ teeth in the mucro. See the comparison among them in remarks of the late species. 


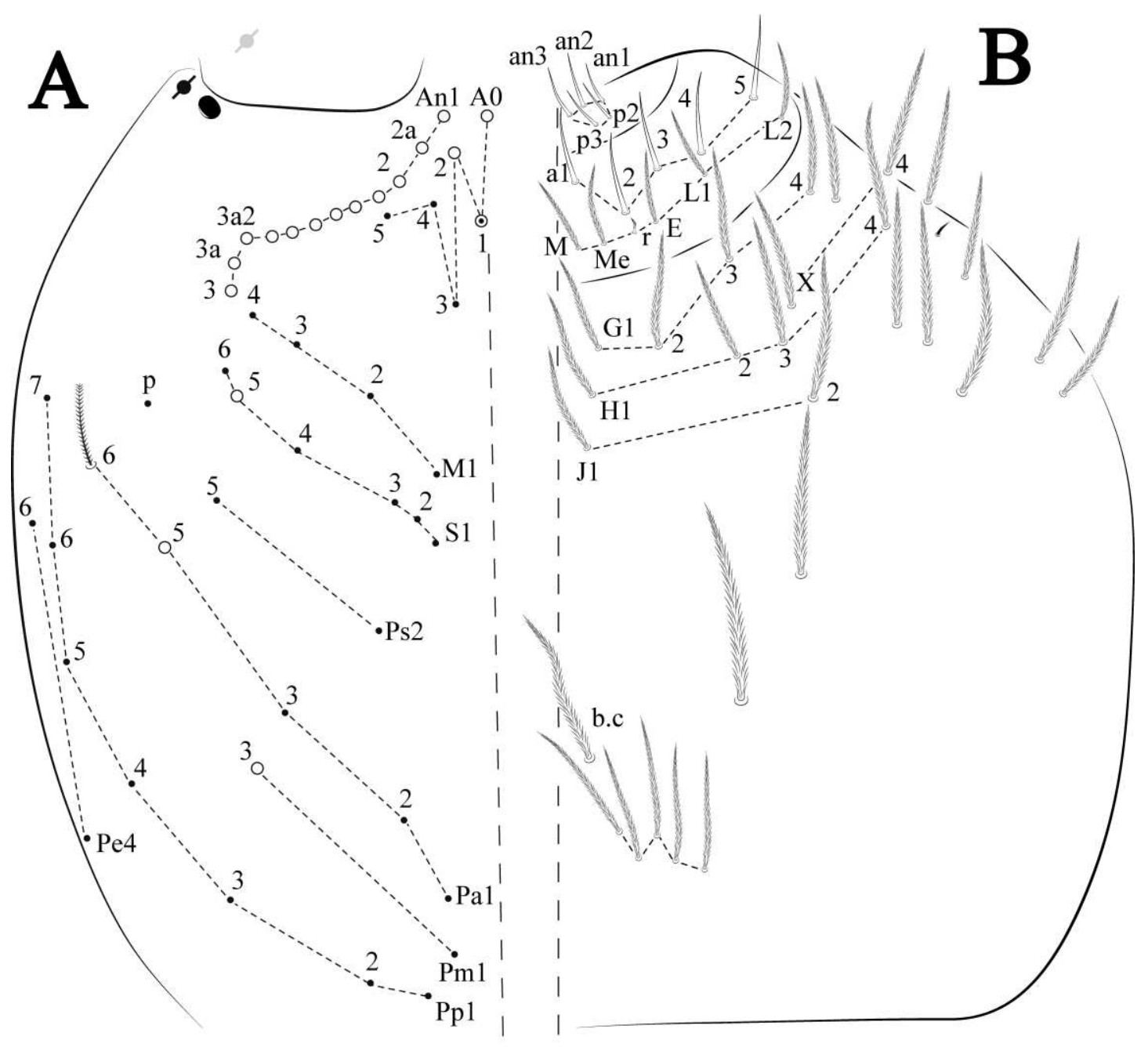

Figure 41. Trogolaphysa dandarae sp. nov.: A) Head dorsal chaetotaxy; B) labial proximal chaetae, basomedial and basolateral labial fields and postlabial chaetotaxy. Black cut circle, pseudopore; Gray cut circle pseudopore at the under surface. 


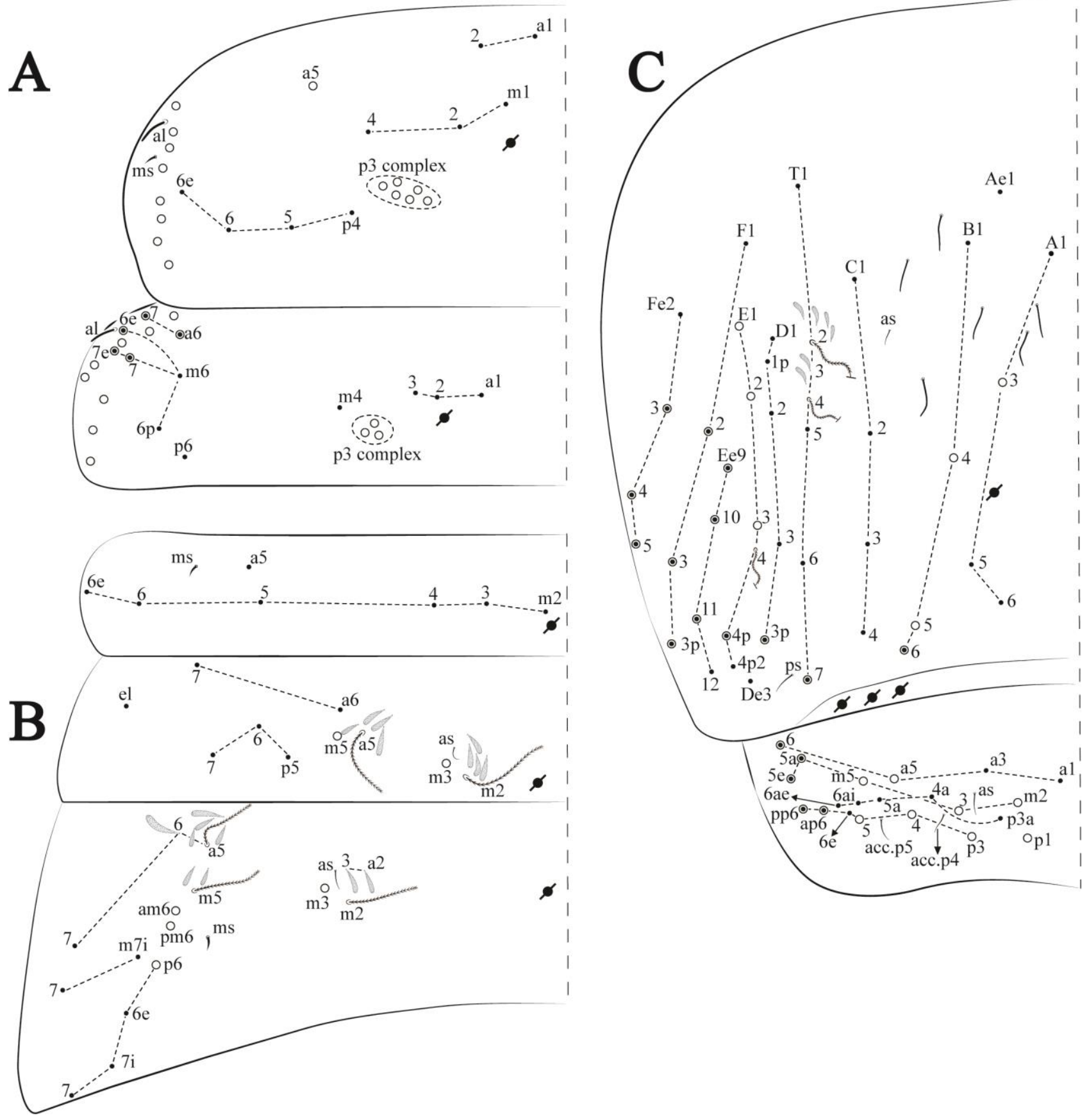

Figure 42. Trogolaphysa dandarae sp. nov.: Dorsal chaetotaxy. A) Th II-III; B) Abd I-III; C) Abd IV-V. 

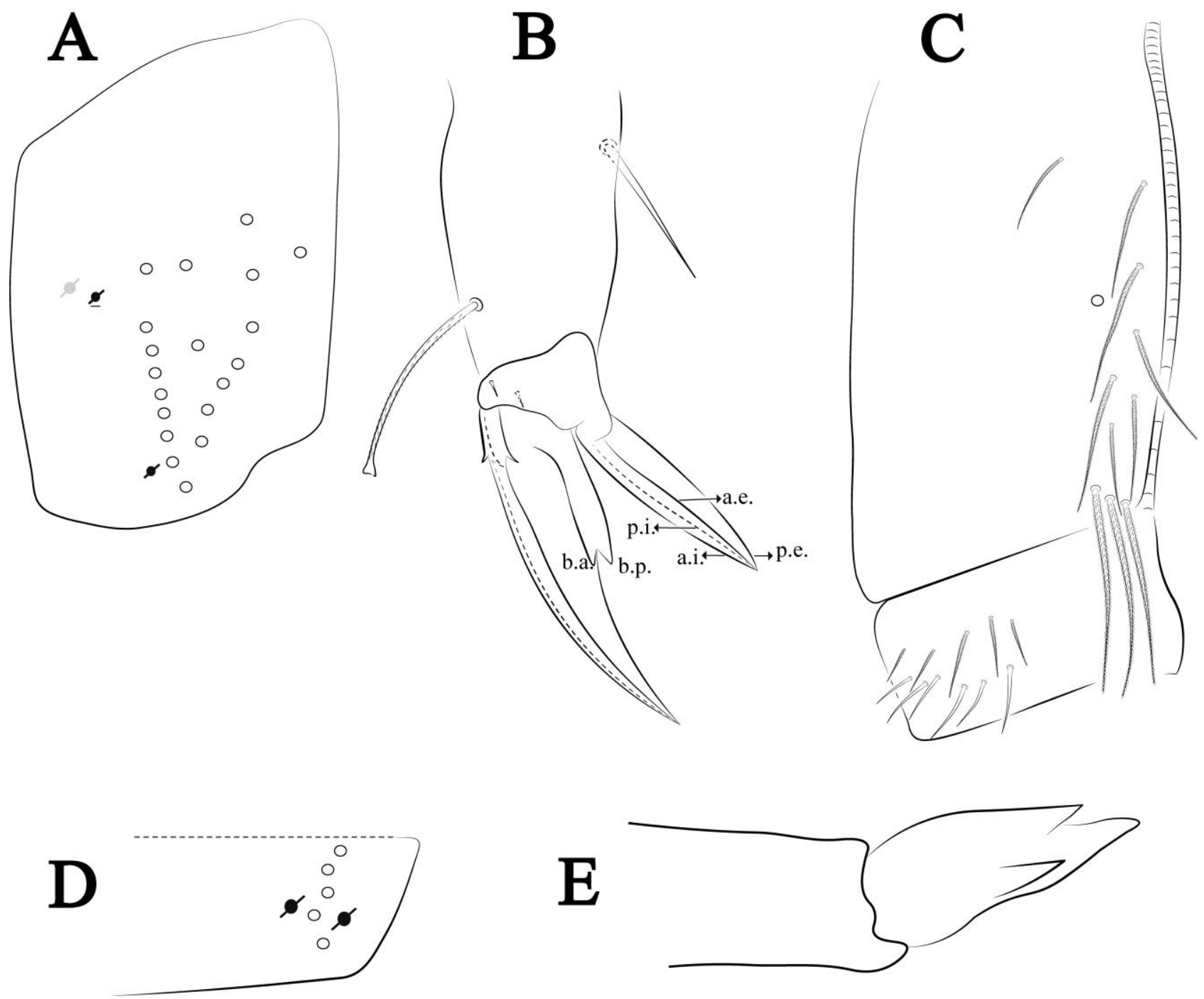

Figure 43. Trogolaphysa dandarae sp. nov.: A) Trochanteral organ; B) Distal tibiotarsus and empodial complex III (anterior view); C) Manubrial plate; D) Antero-lateral view of collophore chaetotaxy; E) Mucro. 


\section{Data availability}

The datasets generated or analyzed during the current study are available from the corresponding author upon reasonable request.

\section{References}

1. Myers, N., Mittermeier, R.A., Mittermeier, C.G., Fonseca, G.A.B. \& Kent, J. Biodiversity hotspots for conservation priorities. Nature 403, 853-858 https://doi.org/10.1038/35002501 (2000).

2. Culver, D.C. \& Sket, B. Hotspots of subterranean biodiversity in caves and wells. J. Cave Karst Stud. 62, 11-17 (2000).

3. Souza-Silva, M. \& Ferreira, R.L. The first two hotspots of subterranean biodiversity in South America. Subterr. Biol. 19, 1-21. https://doi.org/10.3897/subtbiol.19.8207 (2016).

4. Simões, M.H., Souza-Silva, M. \& Ferreira, R.L. Cave physical attributes influencing the structure of terrestrial invertebrate communities in Neotropics. Subterr. Biol. 16, 103-

121. https://doi.org/10.3897/subtbiol.16.5470 (2015).

5. Bento, D.M. et al. Subterranean "oasis" in the Brazilian semiarid region: neglected sources of biodiversity. Biodivers. Conserv. 30, 3837-3857 https://doi.org/10.1007/s10531-021-02277-6 (2021).

6. Juberthie, C., Delay, B. \& Bouillon, M. Sur l'existence du milieu souterrain superficiel en zone non calcaire. C. R. Acad. Sci., Paris 290, 49-52 (1980).

7. White, W.B. \& Culver, D.C. Encyclopedia of Caves. 2th Edition. Elsevier, 966 pp (2012).

8. Ledesma, E. et al. Arthropod biodiversity patterns point to the Mesovoid Shallow Substratum (MSS) as a climate refugium. Zoology 141, 125771.

https://doi.org/10.1016/j.zool.2020.125771 (2020).

9. Souza-Silva, M., Martins, R.P. \& Ferreira, R.L. Cave lithology determining the structure of the invertebrate communities in the Brazilian Atlantic Rain Forest. Biodivers. Conserv. 20, 17131729. https://doi.org/10.1007/s10531-011-0057-5 (2011).

10. Zeppelini, D., Brito, R.A.\& Lima E.C.A. Three new species of Collembola (Arthropoda: Hexapoda) from Central Brazilian shallow caves: side effects of long-term application of environmental law on conservation. Zootaxa 4500(1), 059-081.

https://doi.org/10.11646/zootaxa.4500.1.3 (2018).

11. Cipola, N.G. et al. Review of eyeless Pseudosinella Schaffer (Collembola, Entomobryidae, and Lepidocyrtinae) from Brazilian caves. Insects 11(3), 194.

https://doi.org/10.3390/insects11030194 (2020).

12. Zeppelini, D. The genus Arrhopalites Börner, 1906 (Collembola, Appendiciphora, Arrhopalitidae) in the Neotropical Region, with description of four new cave species from Brazil. Zootaxa 1124, 1-40. https://doi.org/10.11646/zootaxa.1124.1.1 (2006).

13. Gallão J.E. \& Bichuette, M.E. Brazilian obligatory subterranean fauna and threats to the hypogean environment. ZooKeys 746, 1-23. https://doi.org/10.3897/2Fzookeys.746.15140 (2018).

14. Brescovit, A.D. \& Cizauskas, I. Seven new species of the spider genus Matta Crosby from caves in the State of Minas Gerais, Brazil (Araneae, Tetrablemmidae). Zootaxa 4559(3), 401-444. https://doi.org/10.11646/zootaxa.4559.3.1 (2019).

15. Pinto-da-Rocha, R. Sinopse da fauna cavernícola do Brasil (1907-1994). Papéis Avulsos Zoologia 39(6), 61-163 (1995). 
16. Rabelo, L.M., Souza-Silva, M. \& Ferreira, R.L. Priority caves for biodiversity conservation in a key karst area of Brazil: comparing the applicability of cave conservation indices. Biodivers. Conserv. 27(9), 2097-2129. https://doi.org/10.1007/s10531-018-1554-6 (2018).

17. Sket, B. Can we agree on an ecological classification of subterranean animals? J. Nat. Hist. 42, 1549-1563. https://doi.org/10.1080/00222930801995762 (2008).

18. Pipan, T., López, H., Oromí, P., Polak, S. \& Culver, D.C. Temperature variation and the presence of troglobionts in terrestrial shallow subterranean habitats. J. Nat. Hist. 45, 253-273. https://doi.org/10.1080/00222933.2010.523797 (2011).

19. Nae, I. \& Băncilă, R.I. Mesovoid shallow substratum as a biodiversity hotspot for conservation priorities: analysis of oribatid mite (Acari: Oribatida) fauna. Acarologia, 57, 855-868. https://doi.org/10.24349/acarologia/20174202 (2017).

20. Katz, A.D., Taylor, S.J. \& Davis, M.A. At the confluence of vicariance and dispersal: Phylogeography of cavernicolous springtails (Collembola: Arrhopalitidae, Tomoceridae) codistributed across a geologically complex karst landscape in Illinois and Missouri. Ecol. Evol. 8, 10306-10325. https://doi:10.1002/ece3.4507 (2018).

21. Vieira, B.C., Salgado, A.A.R. \& Santos, L.J.C. Landscapes and Landforms of Brazil-World Geomorphological Landscapes. Springer, Dordrecht. 230 https://doi.org/10.1007/978-94-0178023-0 (2015).

22. Jones, W.K. Physical Structure of the Epikarst. Acta Carsologica 42(2-3), https://doi.org/10.3986/ac.v42i2-3.672 (2013).

23. Christiansen, K. Morphological Adaptations. In: Culver, D.C. White, W. (eds) Encyclopedia of Caves. Elsevier Academic Press, USA, pp. 386-397 (2005).

24. Zhang, F. et al. Cryptic diversity, diversification and vicariance in two species complexes of Tomocerus (Collembola, Tomoceridae) from China. Zool. Scri. 43, 393-404 https://doi:10.1111/zsc.12056 (2014).

25. Katz, D.A. Inferring Evolutionary Timescales without Independent Timing Information: An Assessment of "Universal" Insect Rates to Calibrate a Collembola (Hexapoda) Molecular Clock. Genes, 11(10), 1172. https://doi:10.3390/genes11101172 (2020).

26. Rodrigues, A.S.L. et al. Global gap analysis: priority regions for expanding the global protected-area network. BioScience, 54(12), 1092-1100 (2004).

27. Zeppelini, D., Lima, E.C.A., Brito, R.A. \& Soares, G.A. A new species of Pararrhopalites Bonet \& Tellez (Collembola, Symphypleona, Sminthuridae) from iron caves in Brazil. Neotrop. Entomol. 47, 492-501. https://doi.org/10.1007/s13744-017-0569-0 (2018).

28. Jordana, R. Arbea J.I., Simón, C. \& Luciáñez, M.J. Fauna Ibérica. Collembola Poduromorpha. Vol. 8. Museo Nacional de Ciencias Naturales. Madrid 1-807 (1997).

29. Gisin, H. Espèces nouvelles et lignées évolutives de Pseudosinella endogés (Collembola). Mem. Est. Mus. Zool. Univ. Coimbra 301, 1-25 (1967).

30. Zhang, F. \& Pan, Z.X. Homology of labial chaetae in Entomobryoidea (Collembola). Zootaxa 4766(3), 498-500. https://doi.org/10.11646/zootaxa.4766.3.8 (2020).

31. Fjellberg, A. The labial palp in Collembola. Zoologisher Anzeiger, 237, 309-330 (1999).

32. Chen, J.X. \& Christiansen, K.A. The genus Sinella with special reference to Sinella s. s. (Collembola: Entomobryidae) of China. Orient. Insects 27, 1-54. https://doi.org/10.1080/00305316.1993.10432236 (1993).

33. Cipola, N.G. et al. The survey of Seira Lubbock, 1870 (Collembola, Entomobryidae, Seirinae) from Iberian Peninsula and Canary Islands, including three new species. Zootaxa 4458(1), 001-066. https://doi.org/10.11646/zootaxa.4458.1.1 (2018). 
34. Yoshii, R. \& Suhardjno, Y.R. Notes on the Collembolan fauna of Indonesia and its vicinities. Acta Zool. Asiae Orient. 2, 1-52 (1992).

35. Cipola, N.G., Morais, J.W. \& Bellini, B.C. Two new species of Seira Lubbock (Collembola, Entomobryidae, Seirini) from South Brazil. Zootaxa 3793(1), 147-164. https://doi.org/10.11646/zootaxa.3793.1.7 (2014).

36. Hüther, W. New aspects in taxonomy of Lepidocyrtus (Collembola). In: Dallai, R. (ed) 2nd International Seminar on Apterygota, 61-65 (1986).

37. Oliveira, J.V.L.C., Brito, N.P. \& Zeppelini, D. Two New Cyphoderus Nicolet (Collembola: Paronellidae) of the "bidenticulati-group" with Dental Plurichaetosis from Brazil. Neotrop. Entomol. 50, 579-592. https://doi.org/10.1007/s13744-021-00871-5 (2021).

38. Mari-Mutt, J.A. A revision of the genus Dicranocentrus Schött (Insecta: Collembola: Entomobryidae). Agric. Exp. Stn. Bull. 259, 74-77 (1979).

39. Soto-Adames, F.N. Postembryonic development of the dorsal chaetotaxy in Seira dowlingi (Collembola, Entomobryidae); with an analysis of the diagnostic and phylogenetic significance of primary chaetotaxy in Seira. Zootaxa 1683, 1-31. https://doi.org/10.11646/zootaxa.1683.1.1 (2008).

40. Szeptycki, A. Morpho-systematic studies on Collembola. III. Body chaetotaxy in the first instars of several genera of the Entomobryomorpha. Acta Zool. Cracov. 17, 341-372 (1972).

41. Zhang, F. \& Deharveng, L. Systematic revision of Entomobryidae (Collembola) by integrating molecular and new morphological evidence. Zool. Scri. 44, 298-311 (2015).

42. Szeptycki, A. Chaetotaxy of the Entomobryidae and its Phylogenetical Significance. MorphoSystematic Studies on Collembola. Polska Akademia Nauk: Kraków, Poland, Volume IV, 1219 (1979).

43. Zhang, B., Chen T.W., Mateos, E., Scheu, S. \& Schaefer, I. DNA-based approaches uncover cryptic diversity in the European Lepidocyrtus lanuginosus species group (Collembola: Entomobryidae). Invertebr. Syst. 33, 661-670. https://doi.org/10.1071/IS18068 (2019).

\section{Acknowledgements}

R. Zampaulo, R. Andrade, E. Castro, L.M.S.M. Dornellas and M.P.A. Oliveira provided information about specimen's collection, cave localities and characterization. Speleological team of Carste, Spelayon, Bioespeleo, Ativo Ambiental, and R. Bessi, R. Andrade, R. Zampaulo, and L.M. Rabelo, collected the specimens from caves and MSS.

\section{Author contributions}

Senior author contributed to the conception and design of the study, writing the main text, and revision of the whole manuscript. J.V.L.C.O., E.C.A.L., R.A.B., and A.S.F. described and illustrated the new species. M.A.O.N. edited the figures and ordered the additional records. L.C.S., N.P.B. performed the slide preparation and ordering the biological material under analysis. B.C.H.L. organized the biological material and data base. All authors read and approved the final manuscript. 


\section{Funding}

Senior author is granted by CNPq \# 309030/2018-8, J.V.L.C. Oliveira is granted CAPES\# 88882.440374/2019-01. B.C.H. Lopes is granted by CAPES\# 88882.440392/2019-01.

\section{Competing interests}

The authors declare no competing interests.

\section{Additional information}

\section{Supplementary Material:}

S1 - Additional Records for the new species of Trogolaphysa deposited in the Coleção de Referência de Fauna de Solo (CRFS-UEPB).

S2 - References of Taxonomic authorities.

Correspondence and requests for materials should be addressed to D.Z. 


\section{LEGENDS TO FIGURES}

Figure 1. Schematic profile of ferruginous rock cave and limestone rock cave. A) Ferruginous rock - small and shallow caves, abundant roots, reticulated MSS; fauna and energy come from the above ground (solid red arrow), troglobites inhabit the MSS and reach the limits of the cave, troglophiles inhabit the surrounding and the cave, eventually reaching shortly in the MSS (dotted red arrow). B) Limestone rock - large caves, usually not reached by roots, sparse or absent MSS; fauna and energy come through the cave entrance (solid red arrow), troglobites inhabit the deep aphotic zone reaching the aphotic intermediary zone, troglophiles inhabit the surroundings and the cave, eventually reaching the deep aphotic zone. Yellow to black bar represents the light reach.

Figure 2. Trogolaphysa sp.: habitus lateral view. A-B) specimen fixed in ethanol; C-D) SEM photographs.

Figure 3. Trogolaphysa sp. SEM: general body chaetae. A) Antennal chaetae, sensilla and scales: 1macrochaeta with short ciliation, 2- macrochaeta with long ciliation, 3- microchaeta with long ciliation, 4- microchaeta with short ciliation, 5- finger-shaped sens, 6- wrinkly sens, 7- coffee bean shaped sens, 8- rod sens, 9- spine-like sens, 10- Ant IV subapical-organ, 11- lanceolate scale, 12rounded scales; B) Head chaetae and scales: 1- strait macrochaeta with long ciliation, 2- blunt macrochaeta, 3- smooth chaeta, 4- blunt chaeta, 5- strait microchaeta with long ciliation, 6- labial r microchaeta, 7- cephalic anterior scale, 8- cephalic posterior scale; C) Body and appendages chaetae, sens and scales: 1- bothriotrichum, 2- blunt macrochaeta, 3- blunt mesochaeta, 4- dens external ciliate chaeta, 5- smooth microchaeta, 6- blunt microchaeta, 7- fan-shape chaeta, 8- dental spine, 9- 'al' sens, 10- 'ms' sens, 11- lanceolate scale, 12- intersegmental scale.

Figure 4. Trogolaphysa sp. SEM: antenna: A) Ant IV dorsal view; B) Ant IV apex dorsal view, arrow indicates finger-shaped and wrinkly sens; C) Ant IV apex ventral view, left arrow indicates Ant IV subapical-organ, right arrow points to one sensillum type A8; D) Ant II dorsal view, dashed line indicates rod sens; E) Detail of the sensilla of the Ant III apical organ (red); F) Ant I dorsal view spine like sens (arrows indicate the sensilla in red); G) detail of the Ant I basal, arrow indicates psp and antenobasal organ (yellow and red respectively).

Figure 5. Trogolaphysa sp. SEM: head and mouthpart chaetotaxy. A) clypeus; B) dorsal head; C) eyes (red) circled by dashed line, arrow indicates antenobasal organ and psp; D) ventral head; E) maxillary palp and sublobal plate (right side); F) detail of maxillary palp.

Figure 6. Trogolaphysa sp. SEM: thorax and abdomen dorsal chaetotaxy: A) Th II; B) Th III, C) Abd I-II, D) Abd III.

Figure 7. Trogolaphysa sp. SEM: A) Abd IV dorsal chaetotaxy; B) Abd V dorsal chaetotaxy; C) anal pore and male genital papilla.

Figure 8. Trogolaphysa. sp. SEM: empodial complex III A) external lamella of unguis with external teeth (pseudonychia, yellow); B) unguis and unguiculus lateral view, unguis internal lamella with basal, medial and apical teeth (blue, red and yellow respectively), unguiculus with internal and external teeth, tenent hair capitate (white arrow); C) lateral view, unguiculus lamellae, tenent hair acuminate (white arrow).

Figure 9. Trogolaphysa sp. SEM: appendages A) Metatrochanteral organ with pseudopores (alveoli marked in yellow, white arrows indicate pseudopores); B) ventral tube posterior chaetae; C) ventral tube anterior chaetae; D) Tenaculum.

Figure 10. Trogolaphysa sp. SEM: furca. A) manubrial plate pseudopores (yellow); B) anteroapical chaetae of dens; C) dens anterior view; D) mucro.

Figure 11. Trogolaphysa bellinii: A) Head dorsal chaetotaxy; B) labial proximal chaetae, basomedial and basolateral labial fields and postlabial chaetotaxy.

Figure 12. Trogolaphysa bellinii: Dorsal chaetotaxy. A) Th II-III; B) Abd I-III; C) Abd IV-V. 
Figure 13. Trogolaphysa bellinii: A) Trochanteral organ; B) Distal tibiotarsus and empodial complex III (anterior view); C) Manubrial plate; D) Antero-lateral view of collophore chaetotaxy.

Figure 14. Trogolaphysa lacerta: A) Head dorsal chaetotaxy; B) labial proximal chaetae, basomedial and basolateral labial fields and postlabial chaetotaxy.

Figure 15. Trogolaphysa lacerta: Dorsal chaetotaxy. A) Th II-III; B) Abd I-III; C) Abd IV-V.

Figure 16. Trogolaphysa lacerta: A) Trochanteral organ; B) Distal tibiotarsus and empodial complex III (anterior view); C) Manubrial plate; D) Antero-lateral view of collophore chaetotaxy.

Figure 17. Trogolaphysa chapelensis: A) Head dorsal chaetotaxy; B) labial proximal chaetae, basomedial and basolateral labial fields and postlabial chaetotaxy.

Figure 18. Trogolaphysa chapelensis: Dorsal chaetotaxy. A) Th II-III; B) Abd I-III; C) Abd IV-V.

Figure 19. Trogolaphysa chapelensis: A) Trochanteral organ; B) Distal tibiotarsus and empodial complex III (anterior view); C) Manubrial plate; D) Antero-lateral view of collophore chaetotaxy.

Figure 20. Trogolaphysa crystallensis: A) Head dorsal chaetotaxy; B) labial proximal chaetae, basomedial and basolateral labial fields and postlabial chaetotaxy.

Figure 21. Trogolaphysa crystallensis: Dorsal chaetotaxy. A) Th II-III; B) Abd I-III; C) Abd IV-V.

Figure 22. Trogolaphysa crystallensis: A) Trochanteral organ; B) Distal tibiotarsus and empodial complex III (anterior view); C) Manubrial plate; D) Antero-lateral view of collophore chaetotaxy.

Figure 23. Trogolaphysa sotoadamesi: A) Head dorsal chaetotaxy; B) labial proximal chaetae, basomedial and basolateral labial fields and postlabial chaetotaxy.

Figure 24. Trogolaphysa sotoadamesi: Dorsal chaetotaxy. A) Th II-III; B) Abd I-III; C) Abd IV-V.

Figure 25. Trogolaphysa sotoadamesi: A) Trochanteral organ; B) Distal tibiotarsus and empodial complex III (anterior view); C) Manubrial plate; D) Antero-lateral view of collophore chaetotaxy.

Figure 26. Trogolaphysa mariecurieae: A) Head dorsal chaetotaxy; B) labial proximal chaetae, basomedial and basolateral labial fields and postlabial chaetotaxy.

Figure 27. Trogolaphysa mariecurieae: Dorsal chaetotaxy. A) Th II-III; B) Abd I-III; C) Abd IVV.

Figure 28. Trogolaphysa mariecurieae: A) Trochanteral organ; B) Distal tibiotarsus and empodial complex III (anterior view); C) Manubrial plate; D) Antero-lateral view of collophore chaetotaxy.

Figure 29. Trogolaphysa barroca: A) Head dorsal chaetotaxy; B) labial proximal chaetae, basomedial and basolateral labial fields and postlabial chaetotaxy.

Figure 30. Trogolaphysa barroca: Dorsal chaetotaxy. A) Th II-III; B) Abd I-III; C) Abd IV-V.

Figure 31. Trogolaphysa barroca: A) Trochanteral organ; B) Distal tibiotarsus and empodial complex III (anterior view); C) Manubrial plate; D) Antero-lateral view of collophore chaetotaxy.

Figure 32. Trogolaphysa epitychia: A) Head dorsal chaetotaxy; B) labial proximal chaetae, basomedial and basolateral labial fields and postlabial chaetotaxy.

Figure 33. Trogolaphysa epitychia: Dorsal chaetotaxy. A) Th II-III; B) Abd I-III; C) Abd IV-V.

Figure 34. Trogolaphysa epitychia: A) Trochanteral organ; B) Distal tibiotarsus and empodial complex III (anterior view); C) Manubrial plate; D) Antero-lateral view of collophore chaetotaxy.

Figure 35. Trogolaphysa zampauloi: A) Head dorsal chaetotaxy; B) labial proximal chaetae, basomedial and basolateral labial fields and postlabial chaetotaxy.

Figure 36. Trogolaphysa zampauloi: Dorsal chaetotaxy. A) Th II-III; B) Abd I-III; C) Abd IV-V. 
Figure 37. Trogolaphysa zampauloi: A) Trochanteral organ; B) Distal tibiotarsus and empodial complex III (anterior view); C) Manubrial plate; D) Antero-lateral view of collophore chaetotaxy.

Figure 38. Trogolaphysa gisbertae: A) Head dorsal chaetotaxy; B) labial proximal chaetae, basomedial and basolateral labial fields and postlabial chaetotaxy.

Figure 39. Trogolaphysa gisbertae: Dorsal chaetotaxy: A) Th II-III; B) Abd I-III; C) Abd IV-V.

Figure 40. Trogolaphysa gisbertae: A) Trochanteral organ; B) Distal tibiotarsus and empodial complex III (anterior view); C) Manubrial plate; D) Antero-lateral view of collophore chaetotaxy.

Figure 41. Trogolaphysa dandarae: A) Head dorsal chaetotaxy; B) labial proximal chaetae, basomedial and basolateral labial fields and postlabial chaetotaxy.

Figure 42. Trogolaphysa dandarae: Dorsal chaetotaxy. A) Th II-III; B) Abd I-III; C) Abd IV-V.

Figure 43. Trogolaphysa dandarae: A) Trochanteral organ; B) Distal tibiotarsus and empodial complex III (anterior view); C) Manubrial plate; D) Antero-lateral view of collophore chaetotaxy.

\section{LEGENDS TO SUPPLEMENTARY MATERIAL}

S1 - Additional Records for the new species of Trogolaphysa deposited in the Coleção de Referência de Fauna de Solo (CRFS-UEPB).

S2 - References of Taxonomic authorities.

\section{LEGENDS TO TABLES}

Table 1: Collembola species described from caves and MSS in Brazil. Distributional States - Mato Grosso do Sul, MS. Minas Gerais, MG. Para, PA. Parana, PR. Santa Catarina, SC. São Paulo, SP. Lithology - Granitic rock, Gr. Iron rock, Ir. Limestone rock, Li. Sandstone, Sn. Cave ${ }^{1}, \mathrm{MSS}^{2}$, Surface ${ }^{3}$.

Table 2: Trogolaphysa species of the Neotropical Region, comparative morphology. Be, Belize; Br, Brazil; Ec, Ecuador; Ha, Haiti; Ho, Honduras; Me, Mexico. Cv, cave; Lt, Leaflitter, Tp, Termitophile, Ms, Mesovoid shallow substratum. ?, lacking or dubious information.

\section{Authors ORCID}

Douglas Zeppelini 0000-0002-9026-1129

João Victor L. C. Oliveira 0000-0003-0433-5927

Estevam C. Araújo de Lima 0000-0002-1680-4818

Roniere A. Brito 0000-0002-4613-7673

Aila S. Ferreira 0000-0002-7544-3944

Luis C. Stievano 0000-0001-7302-583x

Nathan P. Brito 0000-0001-9030-4339

Misael A. Oliveira-Neto 0000-0001-9249-9088

Bruna C. H. Lopes 0000-0002-8931-2443 


\section{Supplementary Files}

This is a list of supplementary files associated with this preprint. Click to download.

- ZeppelinietalhotspotSciRepSM12.pdf 